\title{
LINEAR OSCILLATORY DYNAMICS OF FLEXOELECTRIC MEMBRANES EMBEDDED IN VISCOELASTIC MEDIA WITH APPLICATIONS TO OUTER HAIR CELLS
}

\author{
BY \\ Milad Abou Dakka \\ A REPORT SUBMITTED TO THE DEPARTMENT OF CHEMICAL ENGINEERING \\ IN PARTIAL FULFILLMENT OF M.ENG DEGREE REQUIREMENTS. \\ DEPARTMENT OF CHEMICAL ENGINEERING \\ FACULTY OF ENGINEERING \\ MCGILL UNIVERSITY \\ MONTREAL, QUEBEC
}

APRIL 2012 


\begin{abstract}
Membrane flexoelectricity is an electromechanical coupling effect between the membrane average curvature and macroscopic electric polarization. Flexolelectricity is a biological actuation mechanism involved in the functioning of hearing. This thesis uses theory and simulation to develop a fundamental understanding of flexolectricity of relevance to hearing processes by integrating membrane elasticity and flexolectricity with viscoelastic processes. Flexoelectric actuation uses an imposed electric field to create membrane bending. In this thesis we model the small amplitude oscillatory dynamics of a membrane immersed in viscoelastic media driven by a small amplitude harmonic electric field. The model is based on the integration of the flexoelectric membrane shape equation applied to a circular membrane attached to the inner surface of a circular capillary and the coupled capillary flow of the contacting viscoelastic phases, such that the membrane flexoelectric oscillations drive periodic viscoelastic capillary flows. The model for curvature dynamics as a function of the electric field is second order in both inputs and outputs and maps into the mechanical Burgers solid model. The material space of the viscoelastic fluid/flexoelectric membrane material system is defined and used to classify and characterize the frequency response of the material system. The frequency response is characteristic of a second order system with a second order input and displays a single resonant peak in the complex curvature and the total power. The amplitude, frequency and width of the power peak, of relevance to the functioning of outer hair cells is dependent on the inertia emerging from the contacting viscoelastic phases and the ratio between the membrane elasticity and the elasticity of contacting liquids. The integrated flexoelectric/viscoelastic model and the novel findings contribute to the ongoing quest for a fundamental understanding of the functioning of outer hair cells.
\end{abstract}




\begin{abstract}
La flexoélectricité de une membrane est un effet d'accouplement électromécanique entre la membrane, la courbure moyenne et la divergence électrique macroscopique. La flexolélectricité est un mécanisme de commande biologique impliqué dans le fonctionne d'audience. Cette thèse utilise la théorie et la simulation pour développer une compréhension fondamentale de "flexolectricity" de pertinence aux processus d'audience en intégrant l'élasticité de membrane et flexolectricity avec les processus de viscoelastique. La mise en action "Flexoelectric" utilise un champ électrique imposé pour créer la membrane qui courbe. Dans cette thèse nous modelons la petite amplitude dynamique oscillatoire d'une membrane immergée dans les médias viscoelastiques motivés par une petite amplitude un champ électrique harmonique. Le modèle est fondé sur l'intégration de l'équation de forme de membrane flexoelectrique s'est appliqué à une membrane circulaire attachée à la surface intérieure d'un capillaire circulaire et le flux capillaire couplé des phases viscoelastiques contactant, tel que les oscillations flexoelectriques de membrane conduisent les flux capillaires viscoelastiques. Le modèle pour la dynamique de courbure comme une fonction du champ électrique est le deuxième ordre dans les données et les productions et les cartes dans les mécaniques de modèle solide (modele Burger). L'espace matériel de la membrane de fluide/flexoelectrique/viscoelastique et le système matériel sont définis et classifiés et la réponse de fréquence du système matériel est caracterise. La réponse de fréquence est la caractéristique d'un deuxième système d'ordre avec une deuxièmes données d'ordre et affiche un sommet résonnant seul dans la courbure complexe et le pouvoir total. L'amplitude, la fréquence et la largeur du sommet de pouvoir, de pertinence au fonctionner de cellules de cheveux extérieures dépend de l'inertie naissante des phases de viscoelastic contactant et la proportion entre l'élasticité de membrane et l'élasticité de contacter des liquides. Le modèle intégré "flexoelectric/viscoelastic" et les conclusions originales contribuent à la quête continuante pour une compréhension fondamentale du fonctionnement des "Outer Hair Cells".
\end{abstract}




\section{Acknowledgements}

The experiences I derived from completing a Masters Degree at McGill have changed my worldview for the better. Many personal and academic areas have developed and I hope they continue to do so well after time may have blurred the finer details of my sojourn in Montreal. My first and sincere gratitudes go to Dr. Alejandro Rey for welcoming me into the Materials Modeling Research Group, for accompanying me through the infancy of my graduate life and for being an important part of my humble history. For the many hours spent helping my growth as a person and my work as graduate student, I am dearly indebted to my colleague and friend, Dr. Edston Emilio Herrera-Valencia. If it were not for a constant presence and an expert collaboration this work could not have progressed so much in such a short span. Emilio and the beautiful Mexico he represents will always be close by. I owe a great thank you to my dear colleague Yogesh Murugesan for being a role model, working tirelessly and for an overall selfless attitude. Thanks are also extended to each and every member in Rey's MMRG past and present that have made a helpful difference, namely Ghoncheh Rasouli Tezangi among others, who maintained and upkept a tidy, organized and welcoming work environment. Though my chapter in Montreal must come to a close and my project at Wong Building surely passed on to a most eager candidate, there will always be a special place for the University and the City that have contributed to my future. Finally, my family has blessed me with the inspiration to pursue my ambitions and with the motivation to continue my progress. For giving me all of this unconditionally, I owe them all that I have.

Montreal, April 4, 2012

Milad Abou Dakka 
To my family - deep and extended

To my future - always before me 


\section{Table of Contents}

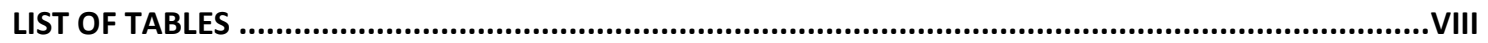

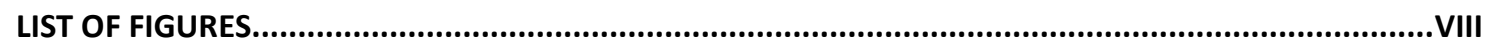

LIST OF NOTATIONS AND ABBREVIATIONS

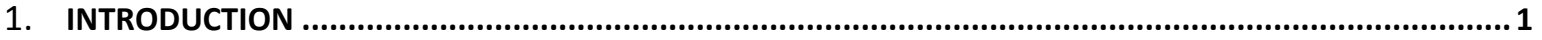

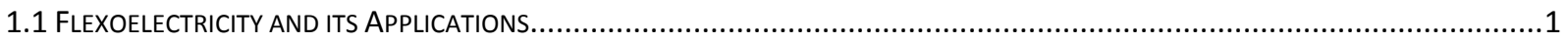

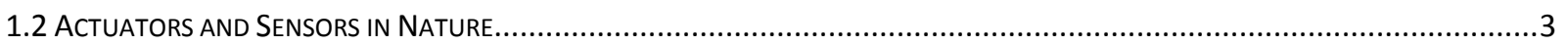

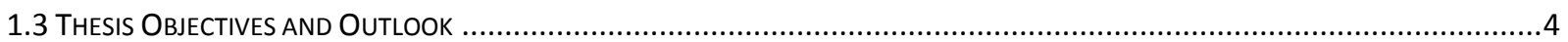

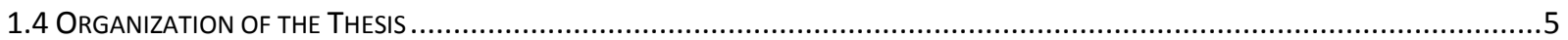

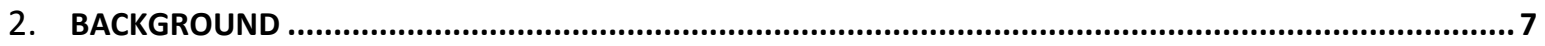

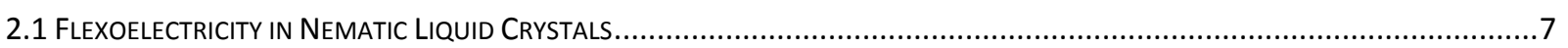

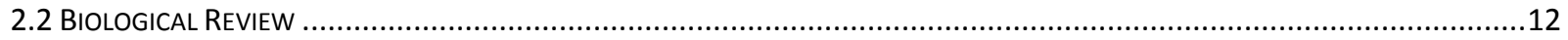

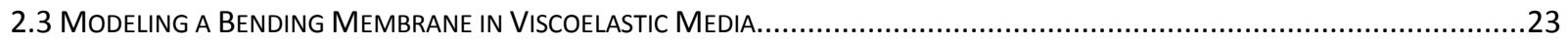

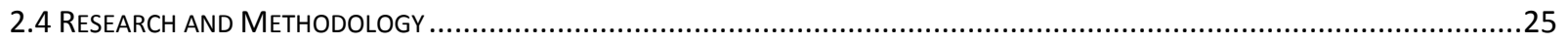

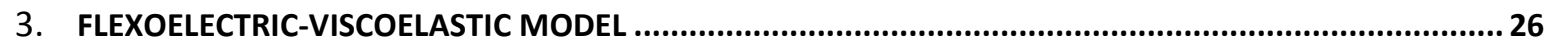

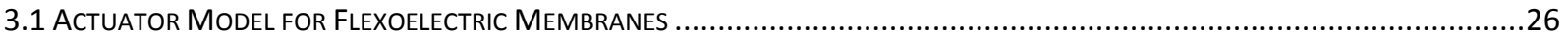

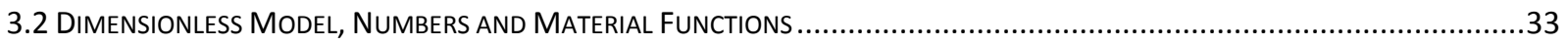

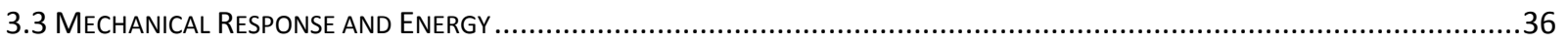

3.4 RESULTS

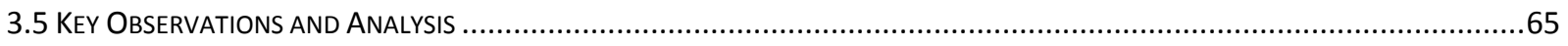

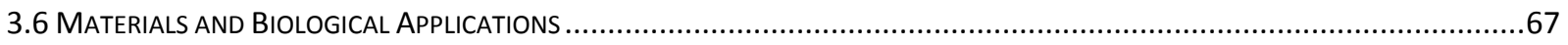

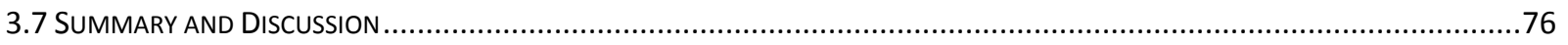

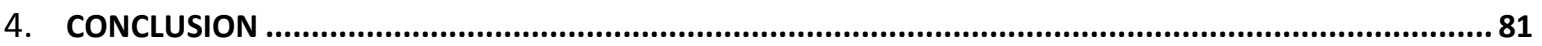

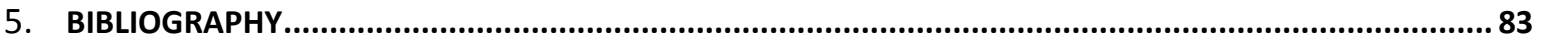

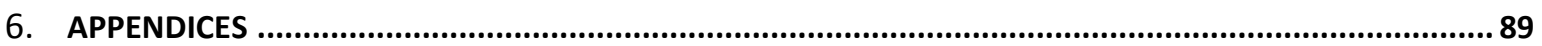

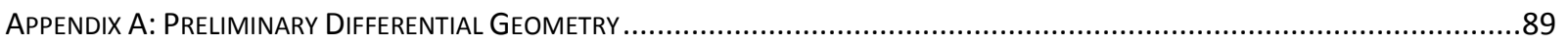

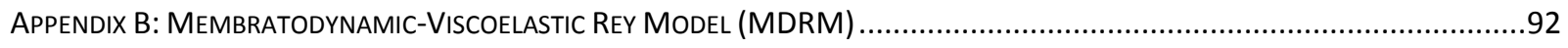

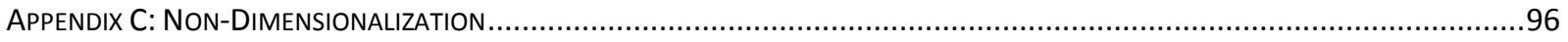

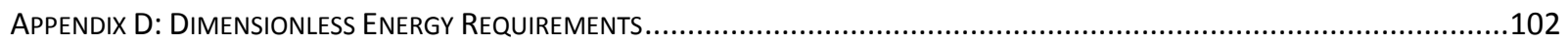




\section{List of Tables}

Table 1 - Model Comparison and Validation

\section{List of Figures}

Figure 1 - Flexoelectric polarization of curved bilayer. Lipids have negative partial charge $\beta e$ and permanent dipole moment $\mu$. The diffuse electric double layer Debye length is $\lambda D, \beta e \lambda D$ and represents the dipole moment per lipid in the double layer, while $d$ is the membrane thickness (Petrov, 1999).

Figure 2 - Illustration of OHC Electromotility: De-/Hydro-polarization (Brownell, 2006).

Figure 3 - Schematic of the geometry and operation of flexoelectric mechanics in a capillary geometry of radius a. Input $\mathbf{E}$ field distorts a flat circular membrane into a spherical cap of radius $\mathrm{R}$ and height $\mathrm{h}$. The flexoelectric actuation creates a capillary viscoelastic flow in the contacting fluids of viscosity $\left\{\eta_{\mathrm{t}}, \eta_{\mathrm{b}}\right\}$ and retardation time $\left\{\lambda_{\mathrm{t}}, \lambda_{\mathrm{b}}\right\}$.

Figure 4 - Illustration of thesis organization. Model of bioinspired mechanotransduction is based on coupling three fundamental concepts shown.

Figure 5 - (Left panel) Flexoelectricity in rod-like and banana-shaped nematic liquid crystals. (Right panel) Flexoelectricity in biological membranes due to bending curvature.

Figure 6 - Schematic of the processes and mechanisms currently accepted to be involved in the functioning of OHC. The electric filed distorts the membrane through the flexoelectric effect. The membrane distortions are transferred to contacting viscoelastic fluids. The combination of flexoelectric actuation and mechanical actuation is flexoelectric mechanics.

Figure 7 - The Ear: Illustration of overall structure of the human ear.

Figure 8 - The Cochlea: The arrows show the direction of propogation of acoustic pressure vibrations.

Figure 9 - Organ of Corti: Radial cochlear cross-sections illustrate the organ of Corti at the structural level between the endolymph-filled scala vestibuli and scala tympani regions (left) and at the cellular level (right).

Figure 10 - Cochlear Hair Cells: Inner (green) and Outer (blue) hair cells are shown in contrast. The ovoid shape of the IHC shows that nature has emphasized its neurotransmitter function while the cylindrical shape of the OHCs (with the nucleus at the basal end of the cell - purple) allows them to perform their roles as tuners by amplifying and enhancing sound vibrations. IHCs are separated from each other by inner phalangeal supporting cells ( $\mathrm{PhC}$ ) while OHCs instead rely on Deiters' supporting cells. Inner and Outer Pillar Cells (IPC, OPC) are also distinguished. 
Figure 11 - Apical Surface of OHC: Shown above through the cuticular plate of the OHC are the stereocilia, forming a bundle. The cytoskeletal layer, called the subsurface cisternae, is held together by a pillar-like lattice.

Figure 12 - The plasma membrane (PM) the cortical lattice and the subsurface cistern (SSC).

Figure 13 - Scheme of a part of two cilias: When the stereocilia bundle tips to a side, the tip link will open a gate channel. The entrance of $\mathrm{Ca}^{2+}$ will activate a motor complex that will use an actin filament to diminish the pressure imposed on the tip link, closing the gate channel.

Figure 14 - Illustration of $\mathrm{OHC}$ bending. $[\mathrm{A}]$ and $[\mathrm{B}]$ show an $\mathrm{OHC}$ hyperpolarized. The cell is longer and the curvature of the plasma membrane of is less than depolarized $\mathrm{OHC}[\mathrm{C}, \mathrm{D}]$.

Figure 15 - (a) Maxwell viscoelastic model and (b) Kelvin-Voigt viscoelastic model.

Figure 16 - Results are understood through 5 key components in our proposed model.

Figure 17 - Schematic of the geometry and operation of flexoelectric mechanics, defined in Figure 2, in a capillary geometry of radius $\mathbf{a}$. The input $\mathbf{E}$ field distorts the initially flat circular membrane into a spherical cap of radius $\mathrm{R}$ and height $\mathrm{h}$. The flexoelectric actuation creates a capillary viscoelastic flow in the contacting fluids of viscosity $\left\{\eta_{\mathrm{t}}, \eta_{\mathrm{b}}\right\}$ and retardation time $\left\{\lambda_{\mathrm{t}}, \lambda_{\mathrm{b}}\right\}$.

Figure 18 - Three dimension material phase diagram for an oscillating flexoelectric membrane in a tube containing viscoelastic fluids. The actuation system is defined by a vector $\mathrm{S}$ (dashed line) whose coordinates $\left(\mathrm{S}_{1}, \mathrm{~S}_{2}, \mathrm{~S}_{3}\right)$ are the membrane elasticity $\mathrm{b}_{0}=\mathrm{M}$, the viscosity function of the bulk viscoelastic fluids $b_{1}(M)$, and the inertia function of the bulk viscoelastic fluids $b_{2}(M)$ respectively.

Figure 19 - (Left) Schematic of material properties in the viscous/elastic plane: In the elasto-viscous plane the operating line $b_{1}(M)$ is: $b_{1}(M)=\Sigma_{\eta}+\Sigma_{\lambda} M$, with slope $\Sigma_{\lambda}$ and abscise $\Sigma_{\eta}$ (see eqn.(18)). (Right) Schematic of material properties in the elasto/inertial plane: the operating line $b_{2}(M)$ is: $b_{2}(M)=X_{\eta \lambda}+\Pi_{\lambda} M$, with slope $\Pi_{\lambda}$ and abscise $X_{\eta \lambda}$ (see eqn.(19)). The operating line in the $M=0$ plane is the relation between inertial $b_{2}$ and viscous $b_{1}$ coefficients: $b_{2}=-X_{\eta \lambda}+\Sigma_{\lambda} b_{1}$ (see eqn. (20)).

Figure 20 - Mechanical response of the curvature moduli, power and elastic energy as a function of the dimensionless frequency. The parametric values are: (i) $\bar{\lambda}_{\mathrm{t}} \bar{\lambda}_{\mathrm{b}}=0.01$, (ii) $\Sigma_{\bar{\eta}}=0.01$, (iii) $\mathrm{k}=0.0054$, and the corresponding dimensionless functions are: (iv) $\mathrm{b}_{1}^{*}=2.84$ and $\mathrm{b}_{2}^{*}=1.85$.

Figure 21 - Shows the mechanical response of the curvature moduli, power and elastic energy as a function of the dimensionless frequency. The dimensionless numbers used in the simulations are: (i) $\bar{\lambda}_{\mathrm{t}} \bar{\lambda}_{\mathrm{b}}=0.01$, (ii) $\Sigma_{\bar{\eta}}=0.01$, (iii) $\mathrm{k}=0.1$, and the corresponding values of the dimensionless functions are: (iv) $b_{1}^{*}=5.5$ and $b_{2}^{*}=0.1$. 
Figure 22 - Shows the mechanical response of the curvature moduli, power and elastic energy as a function of the dimensionless frequency. The dimensionless numbers used in the simulations are: (i) $\bar{\lambda}_{\mathrm{t}} \bar{\lambda}_{\mathrm{b}}=0.01$, (ii) $\Sigma_{\bar{\eta}}=0.5$, (iii) $\mathrm{k}=0.1$, and the corresponding values of the dimensionless functions are: (iv) $b_{1}^{*}=5.5$ and $b_{2}^{*}=5.0$.

Figure 23 - Shows the mechanical response of the curvature moduli, power and elastic energy as a function of the dimensionless frequency. The dimensionless numbers used in the simulations are: (i) $\bar{\lambda}_{\mathrm{t}} \bar{\lambda}_{\mathrm{b}}=0.25$, (ii) $\Sigma_{\bar{\eta}}=0.5$, (iii) $\mathrm{k}=0.1$, and the corresponding values of the dimensionless functions are: (iv) $b_{1}^{*}=5.5$ and $b_{2}^{*}=2.0$, with $b_{1}^{*}>b_{2}^{*}$.

Figure 24 - Shows the $Q$ factor as a function of the dimensionless frequency for different value of the dimensionless parametric dimensionless function $\mathrm{b}_{1}^{*}$. The numerical values employed in the simulation are: a) $\bar{\lambda}_{\mathrm{t}} \bar{\lambda}_{\mathrm{b}}=0.01, \Sigma_{\bar{\eta}}=0.0101$; b) $\bar{\lambda}_{\mathrm{t}} \bar{\lambda}_{\mathrm{b}}=0.01, \Sigma_{\bar{\eta}}=0.9898$; c) $\bar{\lambda}_{\mathrm{t}} \bar{\lambda}_{\mathrm{b}}=0.25, \Sigma_{\bar{\eta}}=0.5$.

Figure 25 - Shows the resonance power versus dimensionless frequency for different values of the dimensionless memory. The numerical values of the dimensionless numbers are: $10^{-2} \leq \mathrm{k} \leq 1, \mathrm{a}$ ) $\bar{\lambda}_{\mathrm{t}} \bar{\lambda}_{\mathrm{b}}=0.1, \Sigma_{\bar{\eta}}=0.0101$; b) $\bar{\lambda}_{\mathrm{t}} \bar{\lambda}_{\mathrm{b}}=0.1, \Sigma_{\bar{\eta}}=0.9898$; c) $\bar{\lambda}_{\mathrm{t}} \bar{\lambda}_{\mathrm{b}}=0.1, \Sigma_{\bar{\eta}}=0.5$; d) $\bar{\lambda}_{\mathrm{t}} \bar{\lambda}_{\mathrm{b}}=0.25$, $\Sigma_{\bar{\eta}}=0.5$.

Figure 26 - Shows the resonance membrane energy versus dimensionless resonance frequency. The numerical values of the dimensionless numbers are: $10^{-2} \leq \mathrm{k} \leq 1$; a) $\bar{\lambda}_{\mathrm{t}} \bar{\lambda}_{\mathrm{b}}=0.1, \Sigma_{\bar{\eta}}=0.0101$; b) $\bar{\lambda}_{t} \bar{\lambda}_{b}=0.1, \Sigma_{\bar{\eta}}=0.9898$; c) $\bar{\lambda}_{t} \bar{\lambda}_{b}=0.1, \Sigma_{\bar{\eta}}=0.5$; d) $\bar{\lambda}_{t} \bar{\lambda}_{b}=0.25, \Sigma_{\bar{\eta}}=0.5$.

Figure 27 - Shows the resonance power as a function of the elastic ratio as a function for different values of the memory of the system $\bar{\lambda}_{\mathrm{t}} \bar{\lambda}_{\mathrm{b}}$. The numerical values of the dimensionless numbers are: a) $\bar{\lambda}_{\mathrm{t}} \bar{\lambda}_{\mathrm{b}}=0.01, \Sigma_{\bar{\eta}}=0.0101$; b) $\bar{\lambda}_{\mathrm{t}} \bar{\lambda}_{\mathrm{b}}=0.01, \Sigma_{\bar{\eta}}=0.9898$; c) $\bar{\lambda}_{\mathrm{t}} \bar{\lambda}_{\mathrm{b}}=0.25, \Sigma_{\bar{\eta}}=0.5$.

Figure 28 - Shows the resonance power as a function of the elastic ratio as a function for different values of the memory of the system $\bar{\lambda}_{\mathrm{t}} \bar{\lambda}_{\mathrm{b}}$. The numerical values of the dimensionless numbers are: a) $\bar{\lambda}_{\mathrm{t}} \bar{\lambda}_{\mathrm{b}}=0.1, \Sigma_{\bar{\eta}}=0.0101$; b) $\bar{\lambda}_{\mathrm{t}} \bar{\lambda}_{\mathrm{b}}=0.1, \Sigma_{\bar{\eta}}=0.9898$; c) $\bar{\lambda}_{\mathrm{t}} \bar{\lambda}_{\mathrm{b}}=0.1, \Sigma_{\bar{\eta}}=0.5$; d) $\bar{\lambda}_{\mathrm{t}} \bar{\lambda}_{\mathrm{b}}=0.25$, $\Sigma_{\bar{\eta}}=0.5$.

Figure 29 - Shows the energy ratio versus dimensionless elastic ratio for different values of the memory. The values used in the numerical predictions are: (i) $\bar{\lambda}_{t} \bar{\lambda}_{\mathrm{b}}=0.01 \cdot \Sigma_{\bar{\eta}}=0.01$; (ii) $\bar{\lambda}_{\mathrm{t}} \bar{\lambda}_{\mathrm{b}}=0.01 ; \Sigma_{\bar{\eta}}=0.9898$; (iii) $\bar{\lambda}_{\mathrm{t}} \bar{\lambda}_{\mathrm{b}}=0.01 ; \Sigma_{\bar{\eta}}=0.5$; (iv) $\bar{\lambda}_{\mathrm{t}} \bar{\lambda}_{\mathrm{b}}=0.25 ; \Sigma_{\bar{\eta}}=0.5$ 
Figure 30 - Dimensionless bulk-viscous number as a function of the elastic ratio. In the inset the power dissipation as a function of the dimensionless frequency. The values used in the numerical predictions are: $\bar{\lambda}_{\mathrm{t}} \bar{\lambda}_{\mathrm{b}}=10^{-4} ; \Sigma_{\bar{\eta}}=0.9999$; (i) $\mathrm{k}=0.50$; (ii) $\mathrm{k}=0.75$; (iii) $\mathrm{k}=0.90$; (iv) $\mathrm{k}=0.99$.

Figure 31 - Dimensionless bulk-viscous number as a function of the elastic ratio. In the inset the power dissipation as a function of the dimensionless frequency. The values used in the numerical predictions are: $\bar{\lambda}_{\mathrm{t}} \bar{\lambda}_{\mathrm{b}}=10^{-4} ; \Sigma_{\bar{\eta}}=0.99999$; (i) $\mathrm{k}=0.50$; (ii) $\mathrm{k}=0.75$; (iii) $\mathrm{k}=0.90$; (iv) $\mathrm{k}=0.99$.

Figure 32 - Shows the elastic membrane energy as a function of the dimensionless frequencies for different values of the elastic ratio. In the Inset, the power dissipation vs dimensionless frequency is showed. The parameters used in the simulation are the same of Figure 31.

Figure $33-Q$ factor vs dimensionless frequency. The material properties used in the simulations are the same as Figures 31-32.

Figure 34 - Schematic representation of the power $\mathbf{P}$ and $Q$ factor as a function of the elastic ratio.

Figure 35 - Rabbitt's [7] model (a) and present model (b).

\section{List of Notations and Abbreviations}

\section{Dimensional Variables}

a

$\left\{a_{i}, b_{i}\right\} ; i=0,1,2 \quad$ Material coeffiecients: bulk viscous, inertial coefficients $\left[1, \mathrm{~s}^{-1}, \mathrm{~s}^{-2}\right]$; Appendix $\mathrm{C}$.

$\left\{\mathrm{C}_{1}, \mathrm{C}_{2}\right\}$

$\mathrm{C}_{\mathrm{i}}(\mathrm{z})$

$\mathbb{C}$

$\mathrm{C}_{\mathrm{f}}$

$\left\{\mathrm{C}_{\mathrm{B}}, \mathrm{C}_{\mathrm{s}}\right\}$

$\left\{\mathrm{E}_{\mathrm{F}}, \mathrm{E}_{\mathrm{H}}\right\}$

Radius of the pipe [m], Appendices B, C

Radius curvature $\left[\mathrm{m}^{-1}\right]$, Appendix $A$

Constant integration $\left[\mathrm{Pa} \mathrm{m} \mathrm{m}^{-3}\right]$, Appendix B

Flexoelectric constant [C], Appendix B

Membrane flexoelectric coefficient [C], Appendix C

Bending and splay flexoelectric coefficients $\left[\mathrm{Cm}^{-1}\right]$

Frank elastic and Helfrich energies $\left[\mathrm{Jm}^{-3}, \mathrm{Jm}^{-2}\right]$ 


\begin{tabular}{|c|c|}
\hline$\left\{\mathrm{E}, \mathrm{E}_{0}\right\}$ & Electrical field magnitude, applied electrical field $\left[\mathrm{NC}^{-1}\right]$; Appendices $\mathrm{B}, \mathrm{C}$ \\
\hline$\{\dot{\mathrm{E}}, \ddot{\mathrm{E}}\}$ & $1^{\text {st }}, 2^{\text {nd }}$ time derivatives of the electrical field $\left[\mathrm{N} / \mathrm{Cs} ; \mathrm{N} / \mathrm{Cs}^{2}\right]$, Appendices $\mathrm{B}, \mathrm{C}$ \\
\hline $\mathrm{F}_{\text {membrane }}$ & Membrane force $[\mathrm{N}]$ \\
\hline$\left\{G_{b}, G_{t}\right\}$ & Elastic moduli define in the bottom and the top $[\mathrm{Pa}]$, Appendices $\mathrm{B}, \mathrm{C}$ \\
\hline h & Height $[\mathrm{m}]$ \\
\hline $\mathrm{H}$ & Average curvature $\left[\mathrm{m}^{-1}\right]$; Appendices $\mathrm{B}$ and $\mathrm{C}$ \\
\hline$\left\{\mathrm{H}_{0}, \mathrm{H}_{1}\right\}$ & First and second initial conditions of the average curvature $\left[\mathrm{m}^{-1}, \mathrm{~m}^{-1} \mathrm{~s}^{-1}\right]$ \\
\hline$\{\dot{\mathrm{H}}, \ddot{\mathrm{H}}\}$ & First/second time derivatives of the curvature $\left[\mathrm{m}^{-1} \mathrm{~s}^{-1} ; \mathrm{m}^{-1} \mathrm{~s}^{-2}\right]$, Appendices $B, C$ \\
\hline$\left\{\mathrm{k}_{\mathrm{c}}, \overline{\mathrm{k}}_{\mathrm{c}}\right\}$ & Membrane bending rigidity and torsion elastic moduli $[\mathrm{J}]$; Appendix $\mathrm{B}$ \\
\hline K & Gaussian curvature $\left[\mathrm{m}^{-2}\right]$, Appendix A \\
\hline$\left\{\mathrm{K}_{1}, \mathrm{~K}_{24}\right\}$ & Splay and saddle constants $[\mathrm{J}]$, Appendix A \\
\hline $\mathrm{L}$ & Axial characteristic length of the capilar [m], Appendices B, C. \\
\hline M & Elastic-membrane parameter $[\mathrm{Pa}]$, Appendices $\mathrm{B}, \mathrm{C}$. \\
\hline$n$ & Exponent of the power law [1], \\
\hline$\left\{\mathrm{P}, \mathrm{P}_{\text {res }}, \mathrm{P}_{\infty}\right\}$ & Power, resonance and infinity power $[\mathrm{J} / \mathrm{s}]$ \\
\hline $\mathrm{P}(\mathrm{z}, \mathrm{t}), \mathrm{P}_{0}, \Delta \mathrm{P}(\mathrm{z}, \mathrm{t})$ & Pressure, constant at $z=0$ and $z=2 L$ and pressure difference $[\mathrm{Pa}] ;$ Appendix $B$ \\
\hline$\left\{\mathrm{P}_{\mathrm{t}}, \mathrm{P}_{\mathrm{b}}\right\}$ & Pressure define in the bottom at top [Pa], Appendix $B$ \\
\hline $\mathrm{Q}(\mathrm{t})$ & Volumetric flow $\left[\mathrm{m}^{3} \mathrm{~s}^{-1}\right]$, Appendix B \\
\hline $\mathrm{R}$ & Spherical cap radius $[\mathrm{m}]$ \\
\hline$(\mathrm{r}, \phi, \mathrm{z})$ & Cylindrical coordinates $[\mathrm{m}, 1, \mathrm{~m}]$, Appendix B \\
\hline
\end{tabular}



$\left(\mathrm{S}_{1}, \mathrm{~S}_{2}, \mathrm{~S}_{3}\right)$
Coordinates of the material point $\mathrm{S}\left[\mathrm{Pa}, \mathrm{Pas} \mathrm{Pas}^{2}\right]$
$\mathrm{t}$
Time variable $[s]$, Appendices $B, C$
$\mathrm{u}$
Parametric variable, Appendix A
$\mathrm{V}_{\mathrm{z}}$
Axial velocity $\left[\mathrm{ms}^{-1}\right]$, Appendix B
$\left\{\mathrm{w}, \mathrm{w}_{\text {res }}\right\}$
Frequency and resonance frequency $\left[\mathrm{s}^{-1}\right]$
$X_{\eta \lambda}$
Cross product of viscosities and Maxwell relaxation times $\left[\mathrm{Pa} \mathrm{s}^{2}\right]$, Appendix C

\section{Vectors and Tensors}

\begin{tabular}{|c|c|}
\hline$\left\{\mathbf{a}_{\alpha}, \mathbf{a}^{\alpha}\right\}$ & Tangential base vectors [1], Appendix A \\
\hline $\mathbf{a}_{\alpha \beta}$ & Surface metric tensor [1], Appendix A \\
\hline b & Symmetric curvature dyadic tensor [1], Appendix A \\
\hline B & Bend orientational deformation $\left[\mathrm{m}^{-1}\right]$ \\
\hline $\mathbf{C}_{\mathrm{f}}$ & Flexoelectric couple stress [] \\
\hline $\mathbf{D}$ & Rate of deformation tensor $\left[\mathrm{s}^{-1}\right]$, Appendix $B$ \\
\hline$\delta_{\alpha}^{\beta}$ & Surface unit tensor [1], Appendix A \\
\hline $\boldsymbol{\varepsilon}$ & Dielectric tensor $\left[\mathrm{Jm}^{-1} \mathrm{~V}^{-2}\right]$ \\
\hline $\boldsymbol{\varepsilon}_{\mathrm{s}}$ & Dyadic surface unit alternator [1], Appendix A \\
\hline$\left\{\mathbf{E}, \mathbf{E}_{/ /}\right\}$ & Electrical and parallel electric field vectors $\left[\mathrm{NC}^{-1}\right]$ \\
\hline $\mathbf{F}_{\mathbf{E}}$ & Electrical force $[\mathrm{N}]$ \\
\hline $\mathbf{F}_{\text {menbrane }}$ & Membrane force $[\mathrm{N}]$ \\
\hline g & Gravitational forces $\left[\mathrm{m} / \mathrm{s}^{-2}\right]$, Appendix B \\
\hline$\left\{\mathbf{I}, \mathbf{I}_{\mathrm{s}}\right\}$ & Dyadic unit and dyadic surface tensor [1] \\
\hline
\end{tabular}




\begin{tabular}{|c|c|}
\hline $\mathbf{k}$ & Outer unit normal vector [1], Appendix A \\
\hline $\mathbf{k k}$ & Dyadic product of the outer unit normal vector $[1]$, Appendix $B$ \\
\hline $\mathbf{n}$ & Director vector [1] \\
\hline $\mathbf{R}$ & Vector position [m], Appendix A \\
\hline $\mathrm{S}_{\mathbf{P}}$ & Splay orientational deformation $\left[\mathrm{m}^{-1}\right]$ \\
\hline $\mathbf{S}$ & Space material vector $\left[\mathrm{Pa}, \mathrm{Pas}, \mathrm{Pas}^{2}\right]$ \\
\hline$\sigma$ & Viscoelastic stress tensor [Pa], Appendix B \\
\hline$\Gamma$ & Torque $[\mathrm{Nm}]$ \\
\hline $\boldsymbol{\Gamma}_{\mathrm{f}}$ & Flexoelectric director $\left[\mathrm{Nm}^{-1}\right]$ \\
\hline $\mathbf{T}_{\mathrm{f}}$ & Flexoelectric stress $\left[\mathrm{Nm}^{-1}\right]$ \\
\hline $\mathbf{P}_{\mathrm{f}}$ & Flexoelectric polarization $\left[\mathrm{JV}^{-1} \mathrm{~m}^{-2}\right]$ \\
\hline $\mathbf{P}$ & Membrane polarization $\left[\mathrm{JV}^{-1} \mathrm{~m}^{-1}\right]$ \\
\hline V & Velocity field $\left[\mathrm{ms}^{-1}\right]$, Appendix B \\
\hline$\Delta \mathbf{T}_{\mathrm{b}}$ & Change of the curvature tensor $\left[\mathrm{m}^{-1}\right]$ Appendix B \\
\hline
\end{tabular}

\title{
Dimensionless Variables
}

\author{
$\left\{b_{2}^{*}, b_{1}^{*}\right\} \quad$ Parametric functions [1], Appendix C. \\ $\{\ddot{\bar{E}}, \dot{\bar{E}}\} \quad$ Second and first time derivatives of the applied electric field [1], Appendix C. \\ $\overline{\mathrm{E}} \quad$ Electrical field [1], Appendices B, C, D. \\ $\overline{\mathrm{Em}} \quad$ Membrane elastic energy [1], Appendices B, D \\ $\overline{\mathrm{Em}}_{\text {res }} \quad$ Elastic membrane energy [1]
}




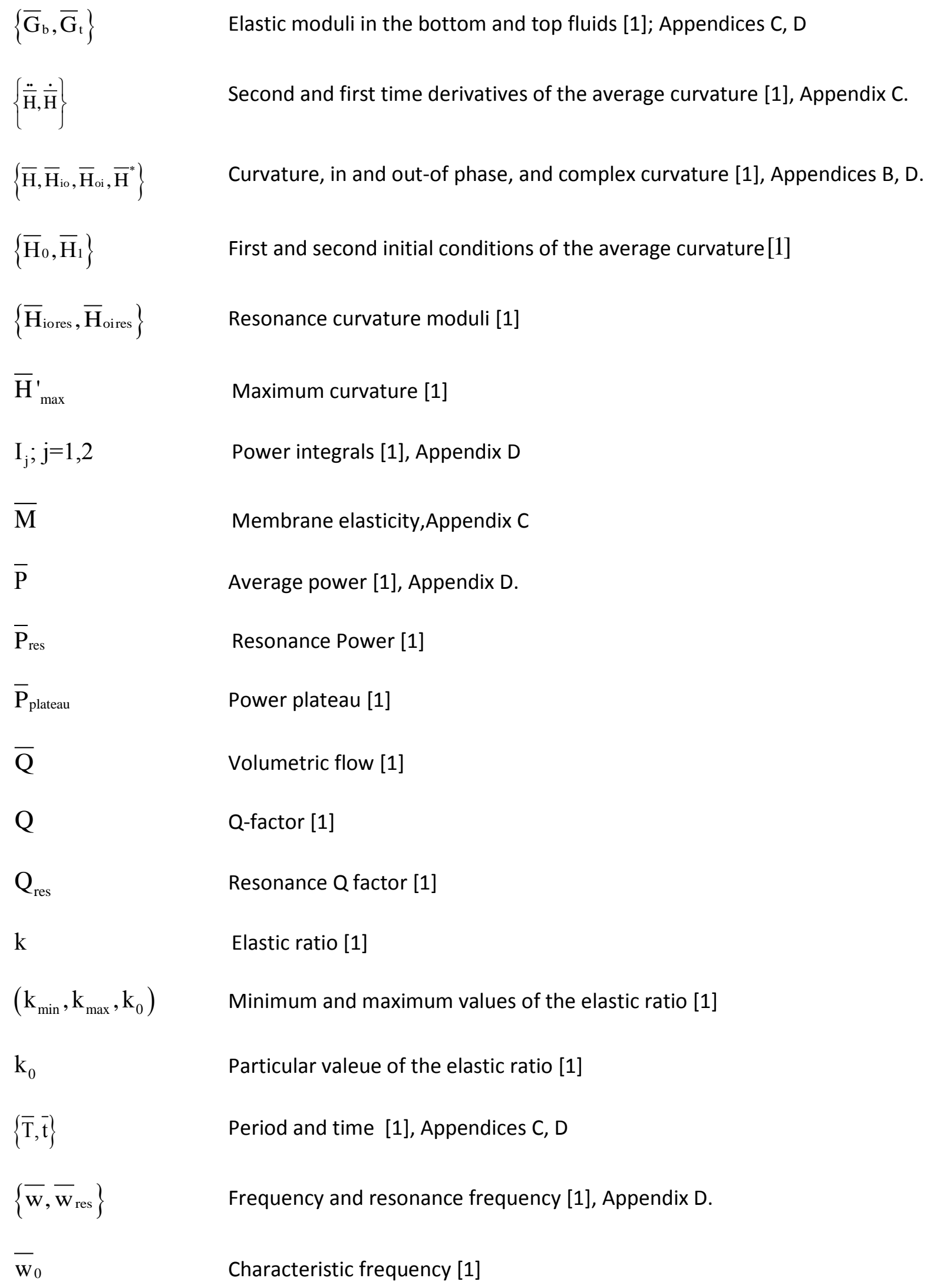



$\overline{\mathrm{W}}_{\text {cross }}$
Cross over frequency [1]
Z
Variable change [1], Appendix D

\section{Greek Letters}
$\in$
Belongs to [1]
$\xi$
Integration variable [s]
$\gamma_{0}$
Interfacial surface tension at 0 electric field [Pa], Appendices B, C
$\dot{\gamma}_{\mathrm{rz}}$
Shear strain $\left[\mathrm{S}^{-1}\right]$; Appendix B
$\left\{\eta_{b}, \eta_{t}\right\}$
Viscosities in the bottom and top [Pa s], Appendix B
$\left\{\lambda_{b}, \lambda_{t}\right\}$
Viscoelastic Maxwell relation times for bottom and the top [s], Appendices B, C
$\rho$
Density of the liquid $\left[\mathrm{kg} / \mathrm{m}^{3}\right]$, Appendix B
$\left\{\Sigma_{\lambda}, \Sigma_{\mathrm{G}}, \Sigma_{\eta=\mathrm{G} \lambda}\right\}$
Sum of Maxwell times, elasticity and viscosities [s,Pa,Pas], Appendix C
$X_{\eta \lambda}$
Cross product between de viscosities and relation times $\left[\mathrm{Pas}^{2}\right]$
$\Pi_{\lambda}$
Product between the viscoelastic times $\left[\mathrm{s}^{2}\right]$, Appendix C.
$\left\{\sigma_{\mathrm{rz}}, \sigma_{\mathrm{rr}}, \sigma_{\theta \theta}, \sigma_{\mathrm{zz}}\right\} \quad$ Components of the shear stress tensor $[\mathrm{Pa}]$, Appendix B

\section{Dimensionless Greek Letters}

$\begin{array}{ll}\Delta \overline{\mathrm{W}} & \begin{array}{l}\text { Discrete change in the dimensionless frequency [1] } \\ \left\{\overline{\lambda_{\mathrm{b}}}, \overline{\lambda_{\mathrm{t}}}\right\}\end{array} \\ \left\{{\overline{\lambda_{\mathrm{b}}}}^{( \pm)},{\overline{\lambda_{\mathrm{t}}}}^{( \pm)}\right\} & \text {Dimensionless Maxwell times for the bottom and top fluids [1], Appendix C } \\ \bar{\eta}_{\mathrm{i}}, \mathrm{i}=\mathrm{t}, \mathrm{b} & \text { Positive and negative roots of the Maxell relaxation times [1] } \\ \Sigma_{\bar{\eta}} & \text { Simensionless viscosities [1], Appendix C }\end{array}$


$\left\{\Sigma_{\bar{\eta} \min }^{( \pm)}, \Sigma_{\bar{\eta} \max }^{( \pm)}\right\}$

\section{Other Symbols}

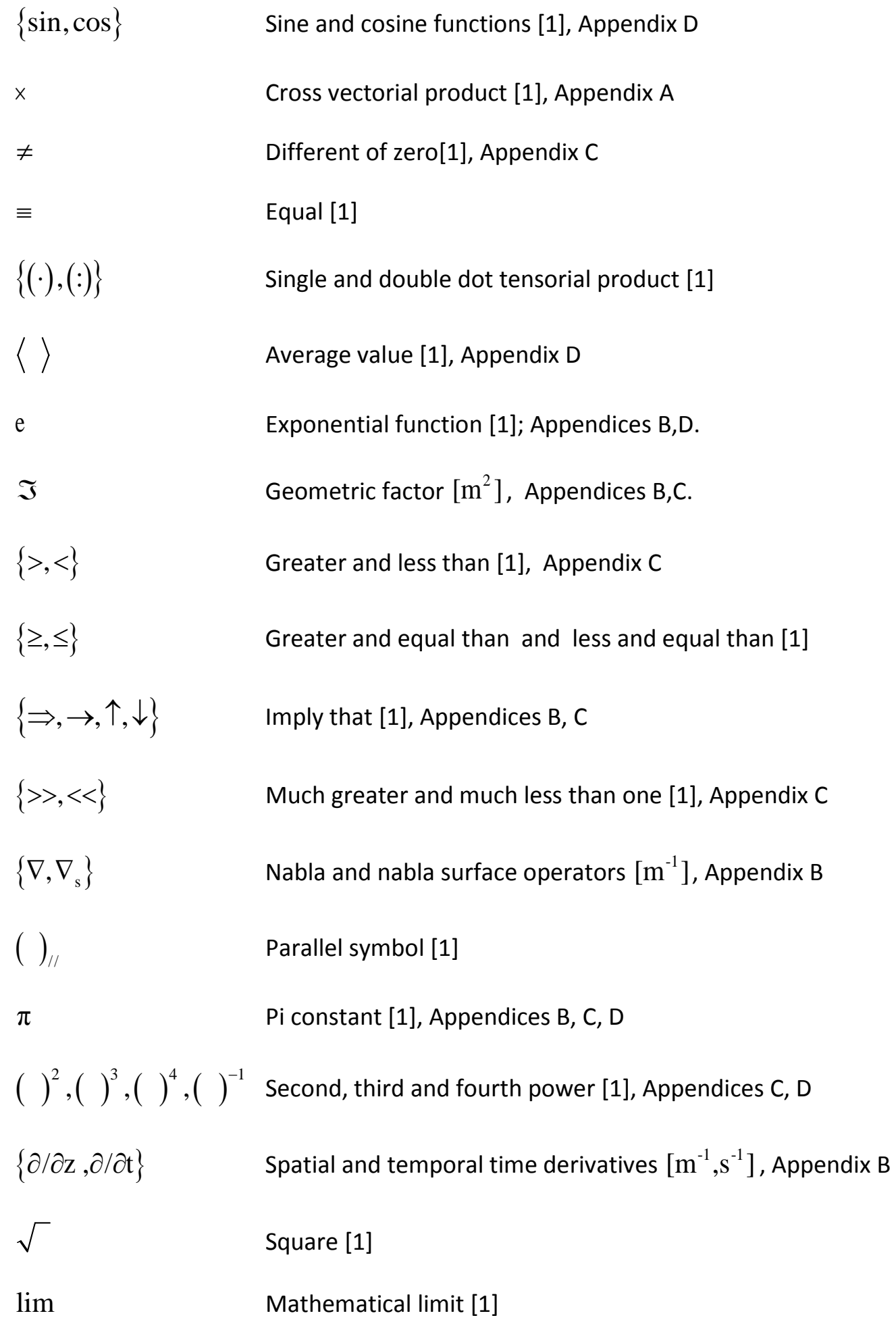

Minimum and maxima total bulk-viscosity values for positive and negative roots corresponding to positive and negative Maxwell relation times [1], Appendix C 

$\mathrm{O}(\mathbf{)}$
Of the order [1], Appendix C
$|\cdot|$
Absolute value [1]
$\operatorname{det}|\cdot|$
determinant [1], Appendix A
$\left\{\int_{0}^{\mathrm{t}}(\cdot) \mathrm{d} \xi, \int_{0}^{\overline{\mathrm{T}}}(\cdot) \mathrm{d} \overline{\mathrm{t}}\right\}$
Define integral [1]; Appendices B, D
$\{\infty, \approx, \infty\}$
Infinity, Approximately, Proportionately [1], Appendices C, D

\section{Subscripts}

Refers to the bottom and the top fluids [1]

$\{\mathrm{rz}, \mathrm{rr}, \theta \theta, \mathrm{zz}\}$

0

Refers to the initial state of the electric and surface tension.

\section{Superscripts}

$=\frac{\mathrm{d}}{\mathrm{dt}}, \cdot=\frac{\mathrm{d}^{2}}{\mathrm{dt}^{2}}$

$\mathrm{T}$

$\nabla$

\section{Abbreviatons}

LC

NLC

$\mathrm{OHC}$

UCME

VEI

EVI

3D
Refers to the first time and second time derivatives $\left[\mathrm{s}^{-1}, \mathrm{~s}^{-2}\right]$, Appendices $B, C$, D

Refers to the transport of a tensor [1], Appendix B

Refers to the upper convective time derivative [1], Appendices A, B

Liquid crystal

Nematic liquid crystal

Outer hair cell

Upper-convective Maxwell equation

Viscoelastic/inertialess

Elasto-visco-inertial

Three dimensional Spaces 


\section{Chapter 1}

\section{Introduction}

Liquid crystals [1] in biology and elsewhere exhibit a form of mechanoelectric coupling known as flexoelectricity - curvature-induced polarization - due to high strain gradients at the nanoscale. Mechanoelectric transduction, indeed at the very core of our hearing system, helps generate mechanical gain from an oscillating membrane. This process happens to counteract the effects of viscous dampening and serves to fine-tune our hearing allowing us to hear with high resolution across a broad spectrum of audio frequencies. Previous work by Petrov [2-6] underpin the mathematics behind the flexoelectricity of such bending lipid bilayer membranes, and while his model assumed inviscid media our integrated approach will make use of and combine a membrane bending model with the Helfrich membrane shape equation in order to study the viscoelastic dynamics of such a membrane.

\subsection{Flexoelectricity and its Applications}

Following many advancements on the effects of curvature on the electrical characteristics of certain compounds, flexoelectricity in a material can now be quantitatively described. Petrov's work provides the mathematical foundation for describing flexoelectricity in lipid bilayers by establishing the theoretical background of the polarization-curvature relationship.

Although its effect tends to be smaller flexoelectricity is actually a more general mechanism than piezoelectricity - electricity resulting from pressure - and surface gradients are large at the nanoscale because the relaxation length scale is short, so small structures of high permittivity 
can have flexoelectric polarizations comparable to those achievable through piezoelectricity (see Figure 1). This has led to interest in all things flexoelectric as higher material coefficients continue to be found.

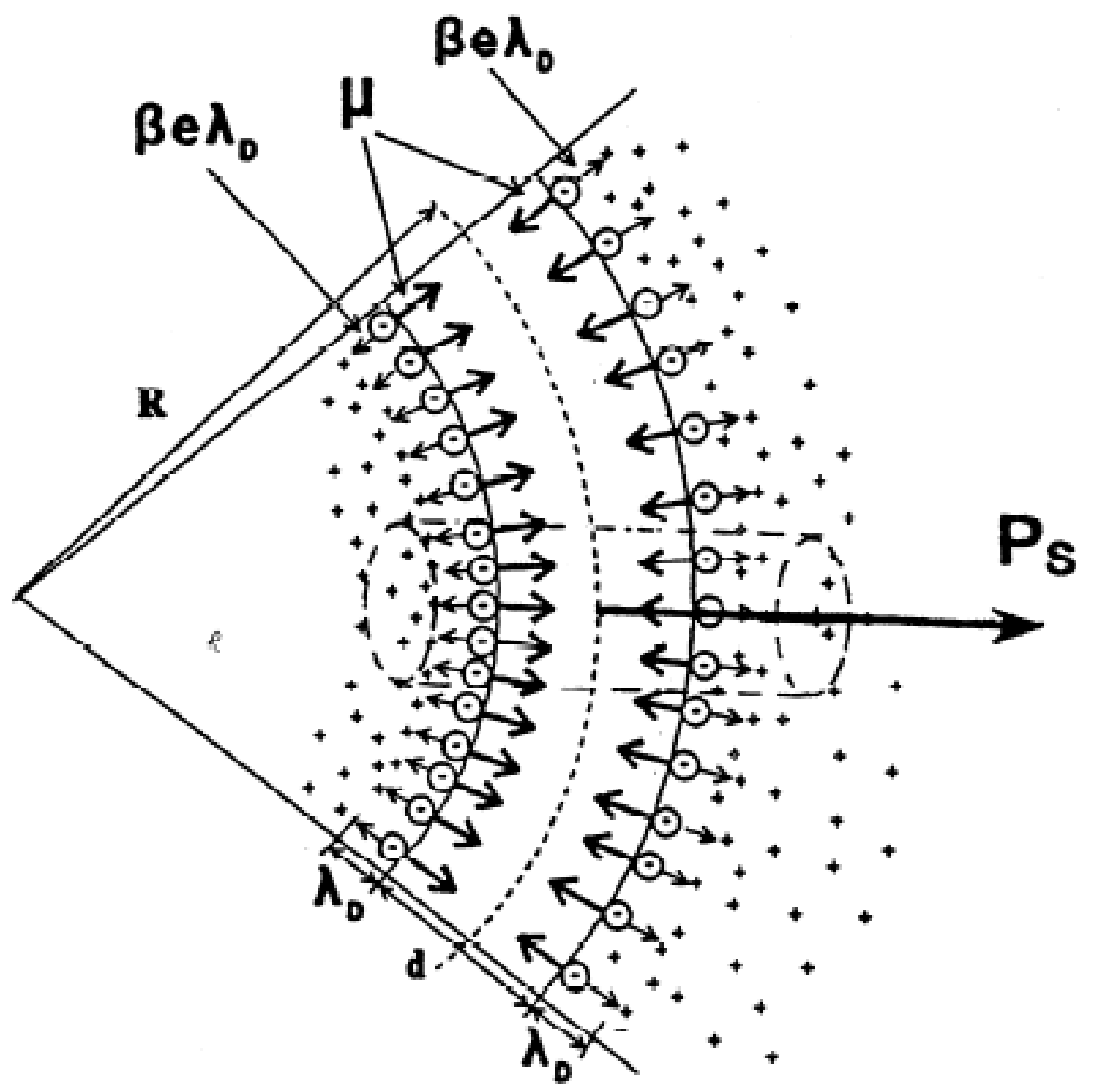

Figure 1 - Flexoelectric polarization of curved bilayer. Lipids have negative partial charge $\beta e$ and permanent dipole moment $\mu$. The diffuse electric double layer Debye length is $\lambda_{D}, \beta e \lambda_{D}$ and represents the dipole moment per lipid in the double layer, while $d$ is the membrane thickness. [4]

From sensors and nanowires to oxides and thin films, this field presents a bright stream of future research in many disciplines linked to this mechanoelectric coupling phenomenon and its applications, which include energy harvesting, lipid-protein electroelastic interactions, curvature thermal fluctuations and elements of blood diffusion and membrane ion transport. 


\subsection{Actuators and Sensors in Nature}

It is not surprising that the study of sensors is a natural one, we all depend on our senses to perceive and interconnect with our surroundings. A prime example is the actuation-sensing biomechanism behind the mammalian hearing system. Spiraling through the human cochlea with a ratio of around 3:1 are 30,000 Outer and Inner Hair Cells (OHC, IHC) responsible for the amplification and detection, respectively, of incoming sound. As shown in Figure 2 from [6] the cylinder-shaped $\mathrm{OHC}$ resonates to electrical inputs extending and contracting in phase with electro-acoustic stimulation to counter the effects of viscosity and significantly power the incoming signal. The IHC is then capable of processing these sounds, otherwise too faint for detection. Different OHCs can vary in height from 10-80 $\mu \mathrm{m}$ providing an astounding hearing range of $10-20,000 \mathrm{~Hz}$.

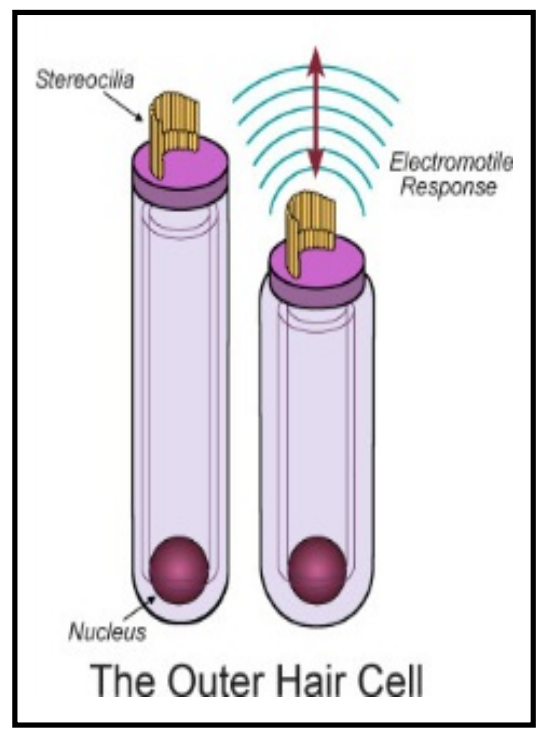

Figure 2 - Illustration of OHC Electromotility: De-/Hyper-polarization.

The phenomenon of electromotility is the inspiration behind our work as we empirically explore the dynamics of an electrically-driven membrane in viscoelastic media and aim to contribute to the ongoing knowledge evolution of the OHC. 


\subsection{Thesis Objectives and Outlook}

This research seeks to contribute to the on-going efforts to characterize flexoelectricity in liquid crystal membranes based on previous actuation models [1-6]. We introduce embedded viscoelastic media and choose a capillary geometry used in the experimental characterization of flexoelectricity, where the oscillating membrane due to an oscillating E field is attached to the wall of the capillary as shown in Figure 3.

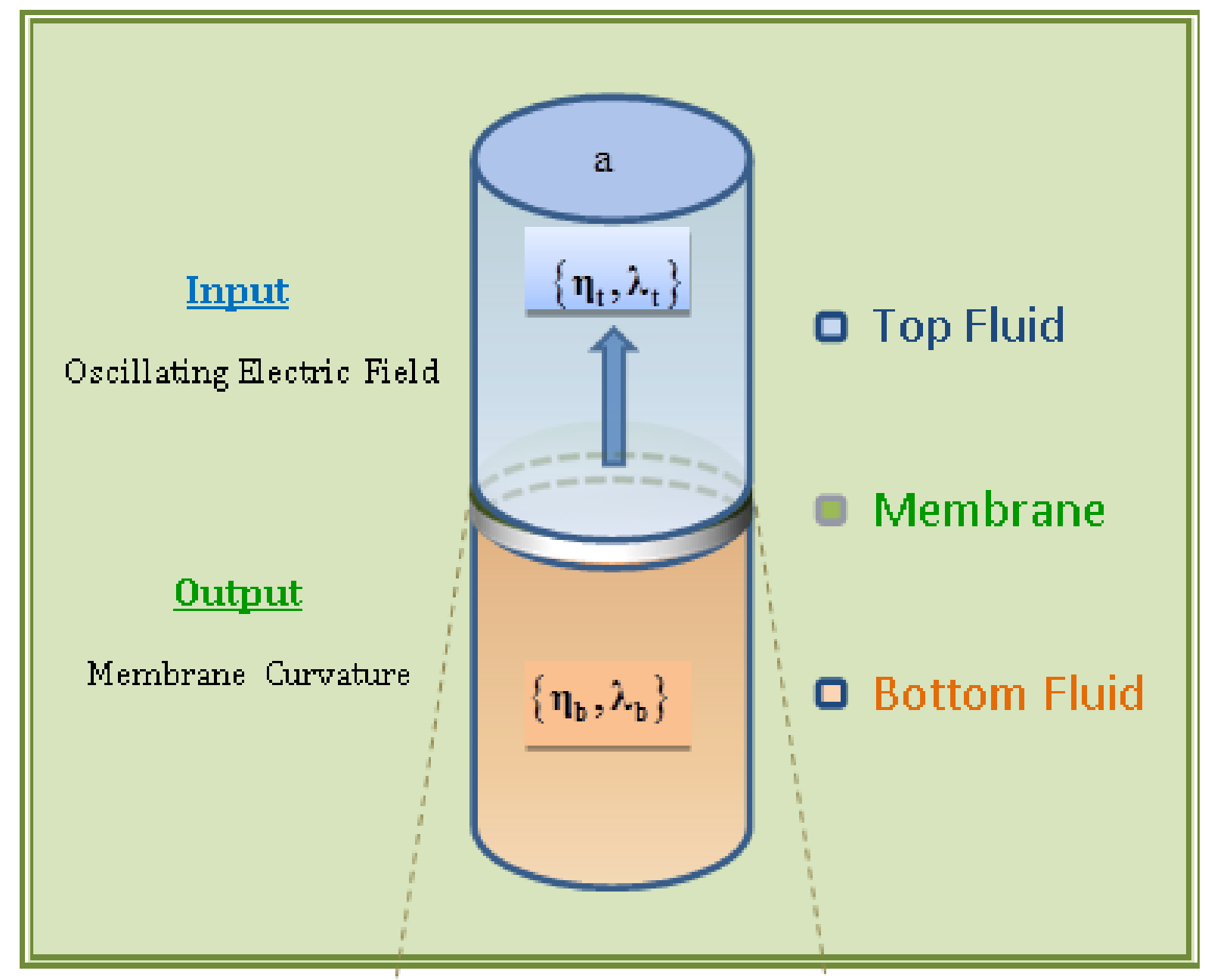

Figure 3 - Schematic of the geometry and operation of flexoelectric mechanics in a capillary geometry of radius a. Input $\mathbf{E}$ field distorts a flat circular membrane into a spherical cap of radius $\mathrm{R}$ and height $\mathrm{h}$. The flexoelectric actuation creates a capillary viscoelastic flow in the contacting fluids of viscosity $\left\{\eta_{\mathrm{t}}, \eta_{\mathrm{b}}\right\}$ and retardation time $\left\{\lambda_{\mathrm{t}}, \lambda_{\mathrm{b}}\right\}$. 
The specific objectives of this thesis are:

1. To derive a dynamic linear model for a flexoelectric membrane attached to a capillary tube that contains viscoelastic liquids and is subjected to a fluctuating small amplitude input electric field of arbitrary frequency;

2. To compute the frequency response of the electromechanical membrane taking into account the viscoelastic nature of the contacting fluids;

3. To compute the viscoelastic functions and power output of the oscillating flexoelectric membrane;

4. To use the modeling results to characterize the role of membrane flexoelectricity and contacting fluid viscoelasticity on the resonance features of the electromechanical oscillator.

Finally, the power efficiency curves found in Rabbitt's [7] model are very important to our research as a validation tool. The resonant behaviour of the $\mathrm{OHC}$ as described will be used as a measuring stick both for comparison and analysis of our own model results.

\subsection{Organization of the Thesis}

In this project we investigate the feasibility of an electric field induced membrane bending model. A tethered membrane, defined using the spherical cap geometry and applied to the Helfrich membrane shape equation, moves between upper and lower viscoelastic fluids in a capillary. This effect is coupled with the force-balance equation for viscoelastic flows and we thus present our results by comparing power and energy results with those of similarly behaving membranes in nature. This thesis conveys the results obtained during the course of this work at the Department of Chemical Engineering, McGill University, Canada. 
In Chapter $\mathbf{2}$ we present an overview of the important background topics including flexoelectricity, the membrane shape equation and biological models of flexoelectric liquid crystals and viscoelasticity. This chapter will serve as the elementary backdrop for understanding the latter results in the thesis.

Chapter 3 presents our results on curvature, energy and power of a membrane with variable stiffness inside media with variable viscoelastic properties. We address comparative models, critical similarities as well relevance and validation.

Chapter 4 summarizes the thesis work and presents an outline of future work as well as considerations on innovative and versatile applications of our technique in the engineering world; with a particular focus on biology.

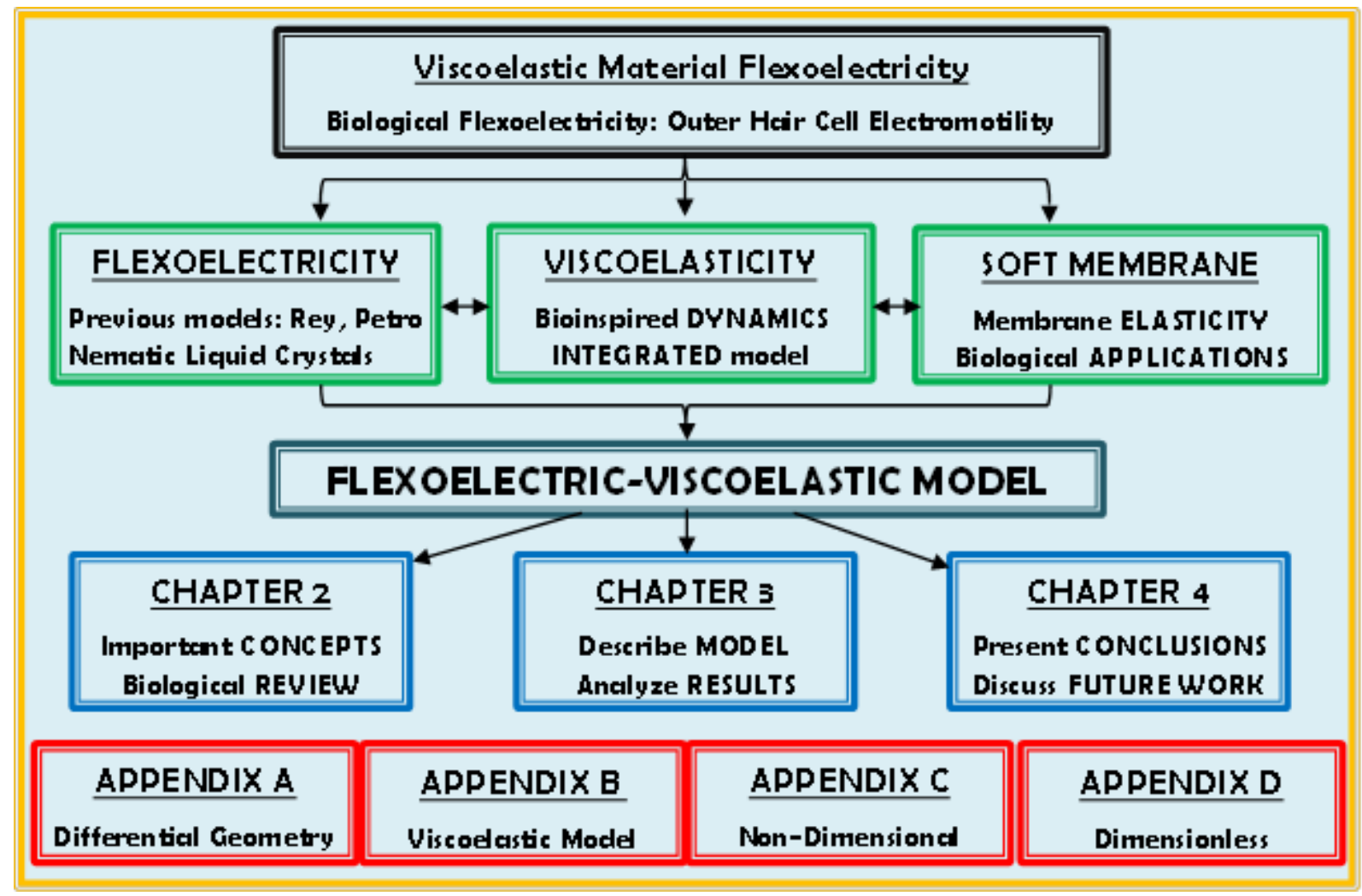

Figure 4 - Illustration of thesis organization. Model of bioinspired mechanotransduction is based on coupling three fundamental concepts shown in green boxes. Blue and red boxes represent the chapters and appendices as well as topics covered within. 


\section{Chapter 2}

\section{Background}

First we recapitulate the multi-disciplinary history of liquid crystal flexoelectricity as it applies to biological membranes and provide an in-depth review of the physiology and function of the Outer Hair Cell $(\mathrm{OHC})$. For in-situ biological considerations several models couple flexoelectricity with viscoelastic behaviour to characterize biomembranes such as the $\mathrm{OHC}$ in their natural environment and in this chapter we go over techniques to couple viscoelastic effects to a curvature-electric field relation, extracting salient points from literature on flexoelectricity and membrane shaping.

\subsection{Flexoelectricity in Nematic Liquid Crystals}

Nematic liquid crystals (NLC) are multifunctional self-organizing viscoelastic anisotropic materials whose orientational order responds to external flow, electromagnetic, chemical, optical and surface fields [1]; the orientational order is defined by the director $\mathbf{n}$ and the elastic

distortions by director gradient $\nabla \mathbf{n}$. A distinguishing and novel property of nematics is flexoelectricity, which describes the coupling between orientational gradients and electric polarization such that an applied electric field creates orientational distortions and distortions create macroscopic polarization. The polar nature of splay $\mathbf{S}=\mathbf{n} \nabla \cdot \mathbf{n}$ and bend $\mathbf{B}=-\mathbf{n} \times \nabla \times \mathbf{n}$ orientational deformations can polarize the NLC medium (see Figure 5):

$$
\mathbf{P}_{\mathrm{f}}=\mathrm{C}_{\mathrm{S}} \mathbf{S}+\mathrm{C}_{\mathrm{B}} \mathbf{B}
$$


$\mathbf{P}_{\mathrm{f}}$ is the flexoelectric polarization and the flexoelectric coefficients for splay and bend $\left\{\mathrm{c}_{\mathrm{s}}, \mathrm{C}_{\mathrm{B}}\right\}$ are of the order of $10 \mathrm{pCm}-1$. Equation 1 describes a sensor or the "direct" flexoelectric effect where the deformation creates polarization. The actuation or "converse" flexoelectric effect [2-5] describes the flexoelectric director torque $\boldsymbol{\Gamma}_{\mathrm{f}}$ due to an electric field $\mathbf{E}$ :

$$
\boldsymbol{\Gamma}_{\mathrm{f}}=\mathbf{n} \times\left\{\left(\mathrm{c}_{\mathrm{S}}-\mathrm{c}_{\mathrm{B}}\right)[\mathbf{E} \nabla \cdot \mathbf{n}-\nabla(\mathbf{n} \cdot \mathbf{E})]+\left(\mathrm{c}_{\mathrm{S}}+\mathrm{c}_{\mathrm{B}}\right) \mathbf{n} \cdot \nabla \mathbf{E}\right\}
$$

where we note that $\boldsymbol{\Gamma}_{\mathrm{f}}$ depends on $\mathbf{E}$ and $\nabla \mathbf{E}$. The torque $\boldsymbol{\Gamma}_{\mathrm{f}}$ is given by the sum of a flexoelectric stress $\mathbf{T}_{\mathrm{f}}$ and flexoelectric coupling stress $\mathbf{C}_{\mathrm{f}}$ :

$$
\boldsymbol{\Gamma}_{\mathrm{f}}=-\boldsymbol{\varepsilon}: \mathbf{T}_{\mathrm{f}}+\nabla \cdot \mathbf{C}_{\mathrm{f}}
$$

This indicates an E field creating a mechanical effect. Current potential applications of LC flexoelectricity include energy harvesting, electro-mechanical transducers, and displays [7-13].

The electroelasticity of synthetic and biological membranes can be efficiently described by liquid crystal models, using an approach denoted by nemato-membranology. For example the elasticity of biological lipid bilayer membranes are well described by the well-known Helfrich energy $E_{H}$ for bending $2 k_{c} H^{2}$ and twisting $\bar{k}_{c} K$ (see Figure 5):

$$
\mathrm{E}_{\mathrm{H}}=2 \mathrm{k}_{\mathrm{c}} \mathrm{H}^{2}+\overline{\mathrm{k}}_{\mathrm{c}} \mathrm{K}
$$

where $\mathbf{H}$ is the average curvature and $\mathbf{K}$ the Gaussian curvature (Appendix A), which follows from the nematic Frank elastic energy $E_{F}[13]$ :

$$
\mathrm{E}_{\mathrm{F}}=\frac{\mathrm{K}_{1}}{2}(\nabla \cdot \mathbf{n})^{2}-\mathrm{K}_{24} \nabla \cdot(\mathbf{n} \nabla \cdot \mathbf{n}+\mathbf{n} \times \nabla \times \mathbf{n})
$$


where $\mathbf{K}_{1}$ is the splay and $\mathbf{K}_{24}$ the saddle splay constants; the geometric quantities and definitions used here are defined in Appendix 1. Nemato-membranology follows by identifying the director $\mathbf{n}$ in relation to the outer unit normal $\mathbf{n} \equiv \mathbf{k}$ and considering the tangential deformation gradient $\nabla_{\mathrm{s}}$ to obtain:

$$
\mathrm{E}_{\mathrm{F}}=\left(\frac{\mathrm{K}_{1}}{2}+4 \mathrm{~K}_{24}\right) \mathrm{H}^{2}+\left(-2 \mathrm{~K}_{24}\right) \mathrm{K}
$$

which coincides with $\mathrm{E}_{\mathrm{H}}$ and gives $4 \mathrm{k}_{\mathrm{c}}=\left(\mathrm{K}_{1}+8 \mathrm{~K}_{24}\right), \overline{\mathrm{k}}_{\mathrm{c}}=-2 \mathrm{~K}_{24}$; the surface gradient is given by the tangential projection of the total gradient: $\nabla_{\mathrm{s}}(\bullet) \equiv \mathbf{I}_{\mathrm{s}} \cdot \nabla(\bullet), \mathbf{I}_{\mathrm{s}}=\mathbf{I}-\mathbf{k k}$. Since thin layers behave like liquid crystals membranes exhibit flexoelectricity. Figure $\mathbf{5}$ shows a schematic of flexoelectric polarization in rod-like and banana-like molecules and the corresponding membrane flexoelectric polarization; as noted above the physics and modeling is affected by relating the director field $\mathbf{n}$ with the membrane normal $\mathbf{k}$.

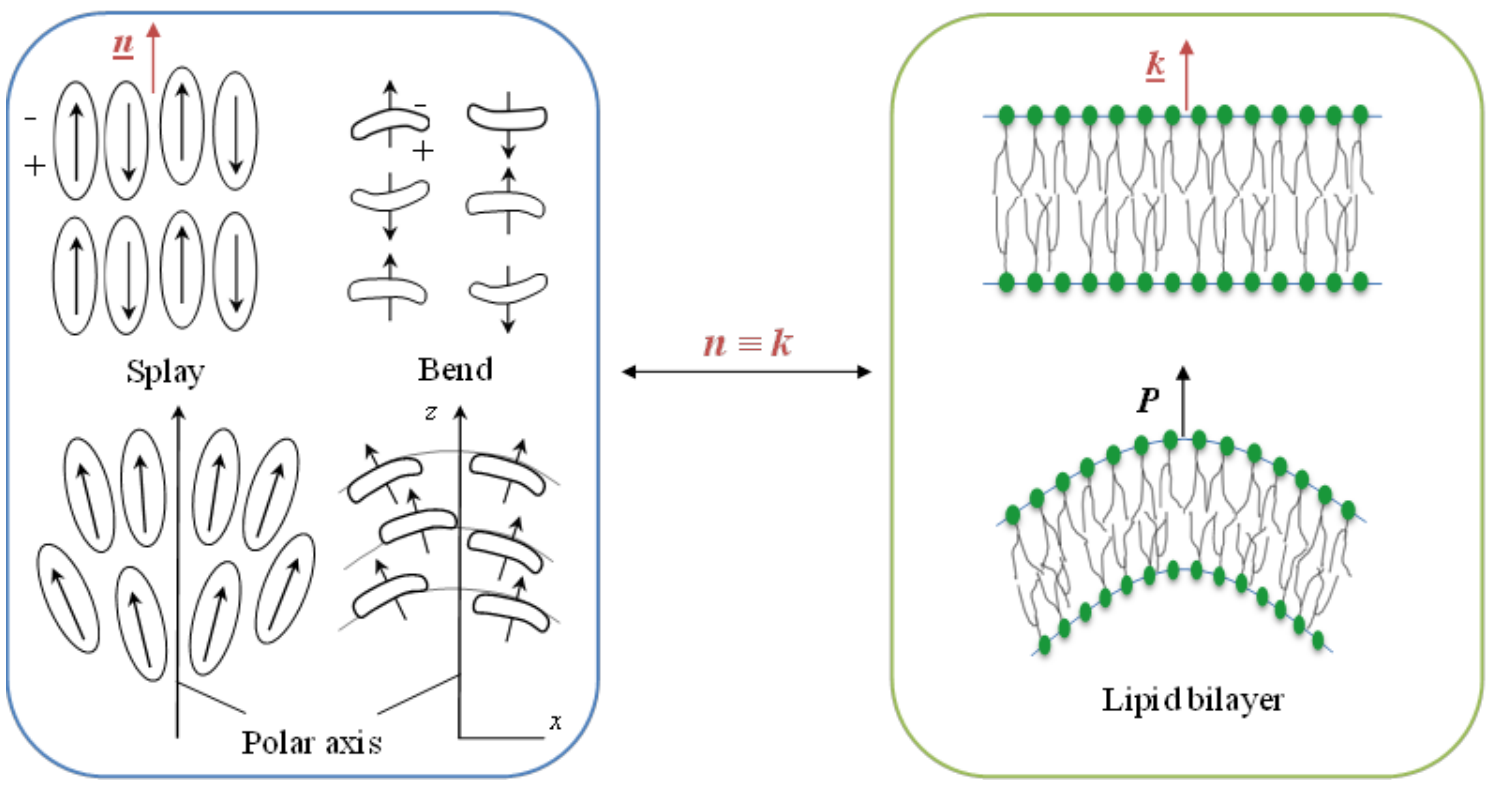

Figure 5 - (Left panel) Flexoelectricity in rod and banana shaped NLC due to splay and bend deformations of the director $\mathrm{n}$. (Right panel) Flexoelectricity in biological membranes due to bending curvature described by surface gradients of the unit normal $\mathrm{k}$. The correspondence between nematic flexoelectricity and membrane flexoelectricity occurs when the director $\mathbf{n}$ is parallel to unit normal $\mathbf{k}$. 
Using the same approach as above, equation (1) gives the membrane polarization $\mathbf{P}$ due to membrane bending $\left(\nabla_{\mathrm{s}} \cdot \mathbf{k}\right)$ :

$$
\mathbf{P}=\mathrm{c}_{\mathrm{f}}\left(\nabla_{\mathrm{s}} \cdot \mathbf{k}\right) \mathbf{k}
$$

where $c_{f}$ is the membrane flexoelectric coefficient, as indeed found experimentally. The converse flexoelectric effect found from equation (2) gives the torque $\boldsymbol{\Gamma}$ due to an imposed electric field $\mathbf{E}$ :

$$
\boldsymbol{\Gamma}=\mathbf{k} \times\left\{\mathrm{c}_{\mathrm{f}}\left[\left(\nabla_{\mathrm{s}} \cdot \mathbf{k}\right) \mathbf{E}_{/ /}-\nabla_{\mathrm{s}}(\mathbf{k} \cdot \mathbf{E})\right]\right\}
$$

where $\mathbf{E}_{/ /}=(\mathbf{I}-\mathbf{k k}) \cdot \mathbf{E}$ is the tangential field. This equation shows that the converse flexoelectric effect in membranes exists when $\mathrm{c}_{\mathrm{f}}>0$, and $\mathbf{E}_{/ /} \neq 0$ or $\nabla_{\mathrm{s}}(\mathbf{k} \cdot \mathbf{E}) \neq 0$.

In partial summary both the direct and converse membrane flexoelectric effects are sensoractuator properties when membrane curvature and polarization are coupled as in nematic liquid crystals. This thesis is motivated by observed flexoelectric actuation in biological membranes, notably in outer hair cells as described below.

Membrane flexoelectricity due to its inherent sensor-actuator capabilities is an area of current interest in soft matter materials [14-21]. Soft matter flexoelectricity complements and extends the performance of piezoelectrics which are based on strain/polarization couplings in hard materials like crystals and ceramics. Many biological complex structures exhibit liquid crystals behavior such as concentrated protein solutions found in spider silk and elsewhere or the phenomenon of electromotility in outer hair cells [22-24]. Over the years much literature has dealt with the problem of measuring flexoelectric coefficients in various liquid crystals and for typical LC membranes these coefficients range from 3-20 pC/m. Recent experiments by Jákli 
and collaborators have reported flexoelectricity coefficients of up to $35 \mathrm{nC} / \mathrm{m}$ in bent-core liquid crystals. Such large bend coefficients make bent-core liquid crystals practical materials for mechanoelectric transduction [25-27].

Observed to behave as a flexoelectric along the lateral walls of their cell membranes, Outer Hair Cells (OHCs) exhibit an actuator-based phenomenon called "electromotility" [28-35]. OHCs produce a gain in the cochlear amplifier of over $50 \mathrm{~dB}$ and the cochlear amplifier provides mammalian physiology with the capacity of "hearing" a wide range of frequencies. Power generation inside the $\mathrm{OHC}$ motor is of interest to the study of hearing loss which in most cases is caused by $\mathrm{OHC}$ deterioration. A key issue in $\mathrm{OHC}$ research is response amplification in the presence of viscous dissipation and elastic storage. Hence an accurate description and understanding of electromotility has to include the frequency response of flexoelectric membranes embedded in a viscoelastic medium due to an oscillating E field [16]. The generic features of this electrical to mechanical energy conversion are described in Figure 6 . The oscillating E field produces oscillations in the elastic membrane through the electromechanical flexoelectric effect. On the other hand the oscillating elastic membrane displaces viscoelastic liquids through the mechanical viscoelasto-elasticity effect. The combined effect that allows the energy conversion is the integration of the flexoelectric effect and the mechanical effect.

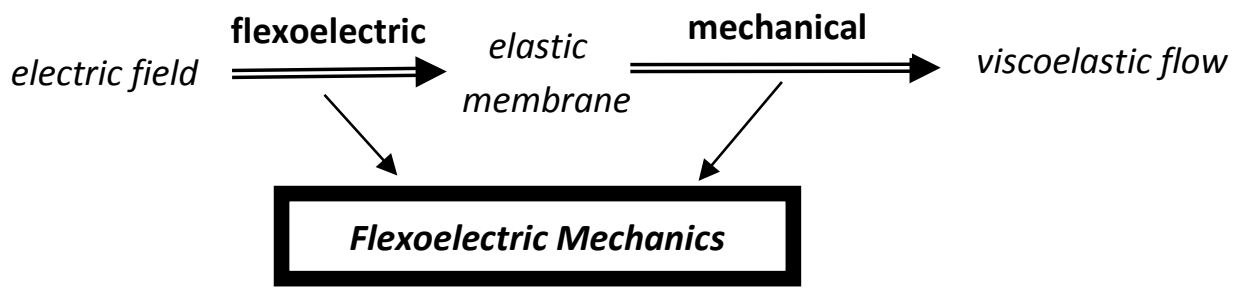

Figure 6 - Schematic of the processes and mechanisms currently accepted to be involved in the functioning of OHC. The electric filed distorts the membrane through the flexoelectric effect. The membrane distortions are transferred to contacting viscoelastic fluids. The combination of flexoelectric actuation and mechanical actuation is flexoelectric mechanics. 


\subsection{Biological Review}

Given the complex nature of the biological process of hearing, we will pursue herein only an overview of the pertinent functions and workings of the ear and in particular that of the inner ear containing the cochlea and $\mathrm{OHC}$.

Implicitly, the ear is divided into parts known as the outer-, middle-, and inner- ear regions (Figure 7). The outer ear contains the pinna and lobule - the visible region of the ear, the external ear canal and the tympanic membrane - or eardrum. The middle ear contains three tiny bones known as the ossicles - malleus, incus and stapes. Finally, the inner ear contains the vestibular apparatus and the cochlea.

A longitudinal sound wave is trapped by the pinna and channeled through the external auditory canal into the vibrating tympanic membrane causing the Ossicle Chain - Malleus, Incus and Stapes - to oscillate accordingly. These oscillations are felt at the oval window, propagating information in the form of fluid vibrations through the hollowed cochlea.

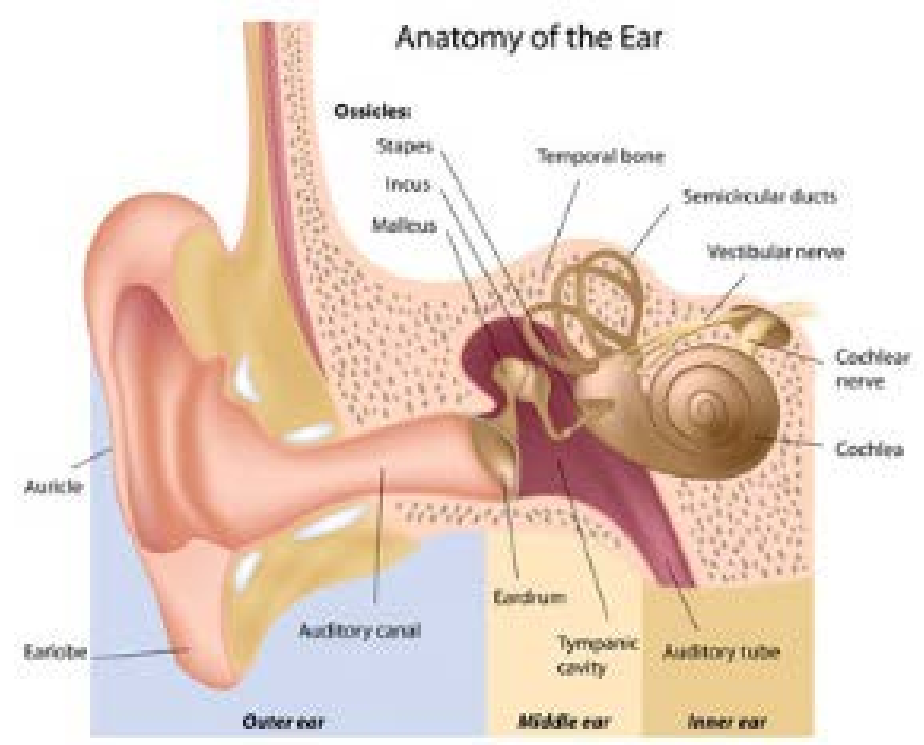

Figure 7 - The Ear: Illustration of overall structure of the human ear. 
Hair cells along the insides of the winding coil react specifically to unique frequencies and relay the information to the primary auditory cortex where in the comfort of the brain's huge processing power the complex sounds of nature are ultimately analyzed.

In order to develop working models at the hair cell level, it is important to understand the behaviour of the inner ear at the cochlear level. A common proverb when studying biological mechanisms, "structure defines function", drives us to examine the inner cochlea and to begin to understand both its function and potentially underlying causes of abnormalities induced by damage or degenerative diseases.

As a small cavity in the temporal bone, the cochlear interior is a mostly hollowed portion of the ear starting at the oval window and spiralling into a close 2.5 turns later at around $5 \mathrm{~mm}$ in height as depicted in Figure 7 from [36]. This provides the spirit of its nomenclature originally derived from the Greek kokhlias - snail, screw, or kokhlos - spiral shell.

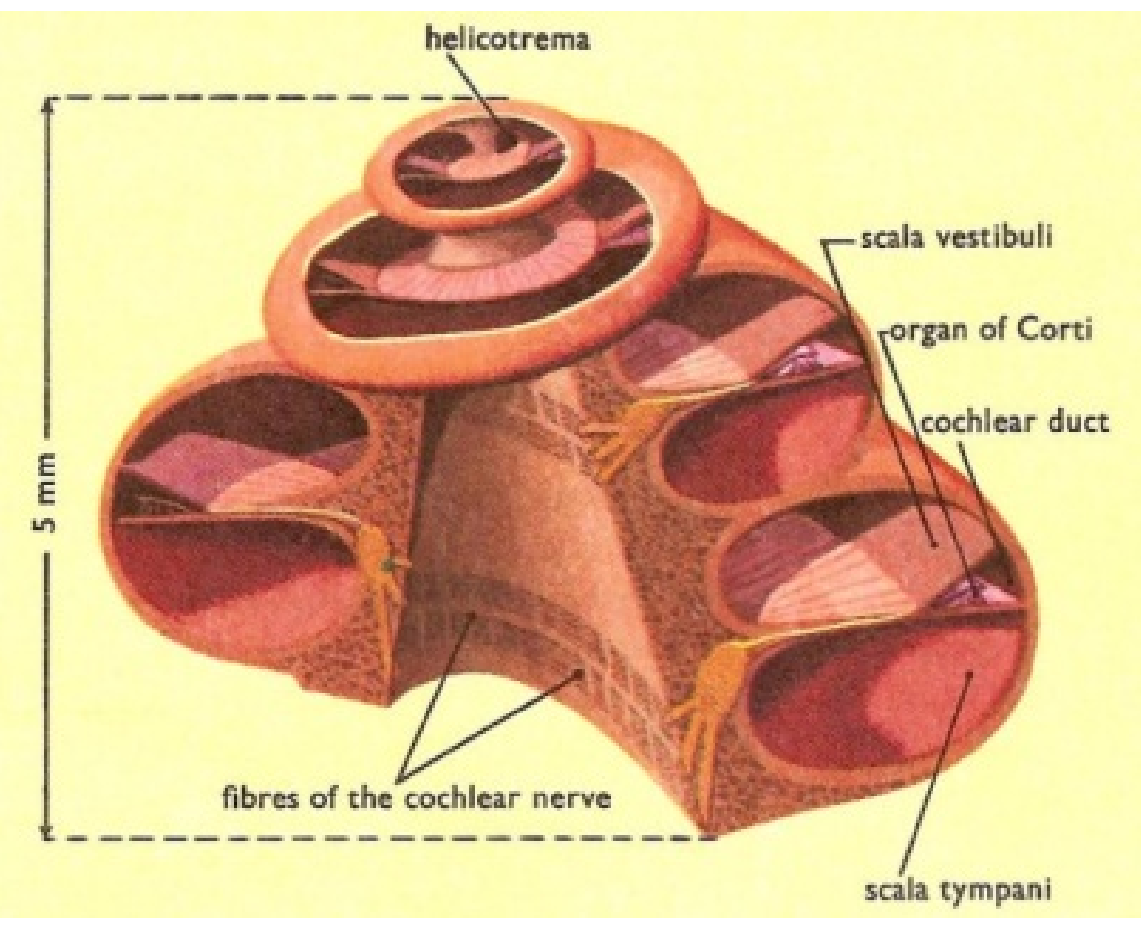

Figure 8 - The Cochlea: The arrows show the direction of propogation of acoustic pressure vibrations. 
Zooming into the cochlea provides a more detailed radial cross-section of the cochlea showing inner components including the sensory organ for hearing, the organ of Corti. Distributed along the scala media, a partition separates the top and bottom fluid chambers coiling around the cochlear duct. With 15,000-20,000 auditory nerve receptors, the Organ of Corti senses the fluid-borne vibrations in the neighbouring scala vestibuli and scala tympani regions, which contain the same fluid called perilymph and actually link together at the apex of the spiral shell called the helicotrema shown in Figure 8. These receptors, each with their own hair cell, are collectively responsible for converting the pressure signals of sound into electrical signals that are ultimately sent to the brain for analysis.

As shown in Figure 9 from [36-37] on the next page, the organ of Corti is further subdivided into two regions between the outer wall of the cochlea lined by the basilar membrane and the inner components including the auditory nerve through to the tectorial membrane, floating atop the organ in the endolymph-rich region of the scala media. On the external side of the organ can be found the Deiters' cells, which connect the basilar membrane to the Outer Hair Cells (OHCs) and that in conjunction with Hensen, Claudius and Boettcher cells provide the support structure for the organ of Corti within the cochlea. The triangular space delimiting the external and internal regions is called the Corti tunnel, filled by a liquid called the cortilymph. The inner section of the organ or Corti includes the auditory nerve fibres, the tectorial membrane as well as the Inner Hair Cells (IHCS), which are directly connected to the auditory nerve fibres and collectively serve as the launching pad for signals sent through the nerves. 

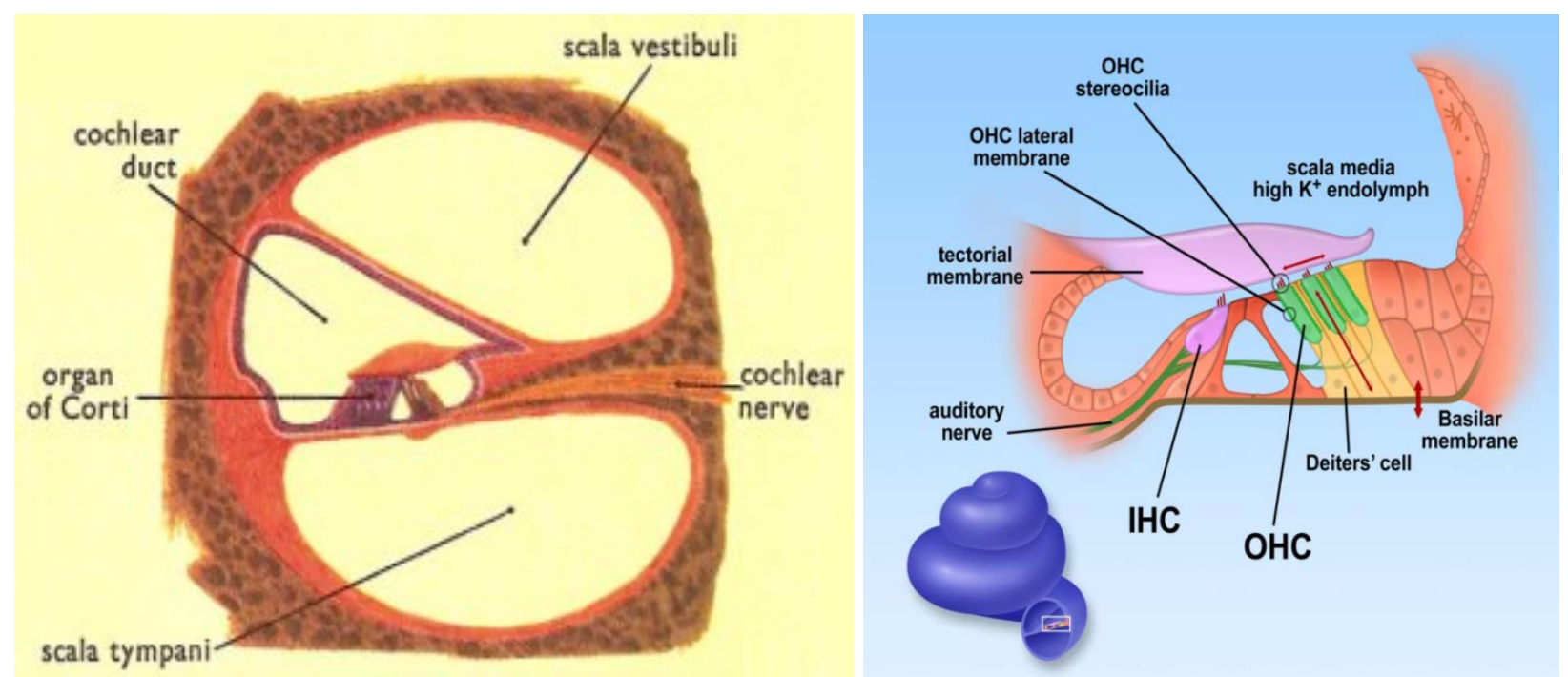

Figure 9 - Organ of Corti: Radial cochlear cross-sections illustrate the organ of Corti at the structural level between the endolymph-filled scala vestibuli and scala tympani regions (left [36]) and at the cellular level (right [37]).

In a human cochlea there up to 30,000 hair cells at birth with a $3: 1$ ratio of Outer to Inner Hair Cells. While IHCs are responsible for detecting and converting vibrations into electrical signals, the OHCs' role is to amplify sounds that are above the sensitivity threshold of the IHC and also to fine tune the resonance of incoming vibrations.

The IHC work with the $\mathrm{OHC}$, sensing pressure vibrations through their own stereocilia bundles arranged in a linear fashion. In contrast with the OHCs, which line entire the organ of Corti in three continuous rows of cells, there is only one row of IHCs lining the basilar floor of the organ of Corti as it spirals up and down the cochlea. The IHC converts the mechanical signals detected at their apical surfaces into electrical signals sent through their basal surfaces, containing their nuclei, along the auditory nerve and finally through to the brain. 


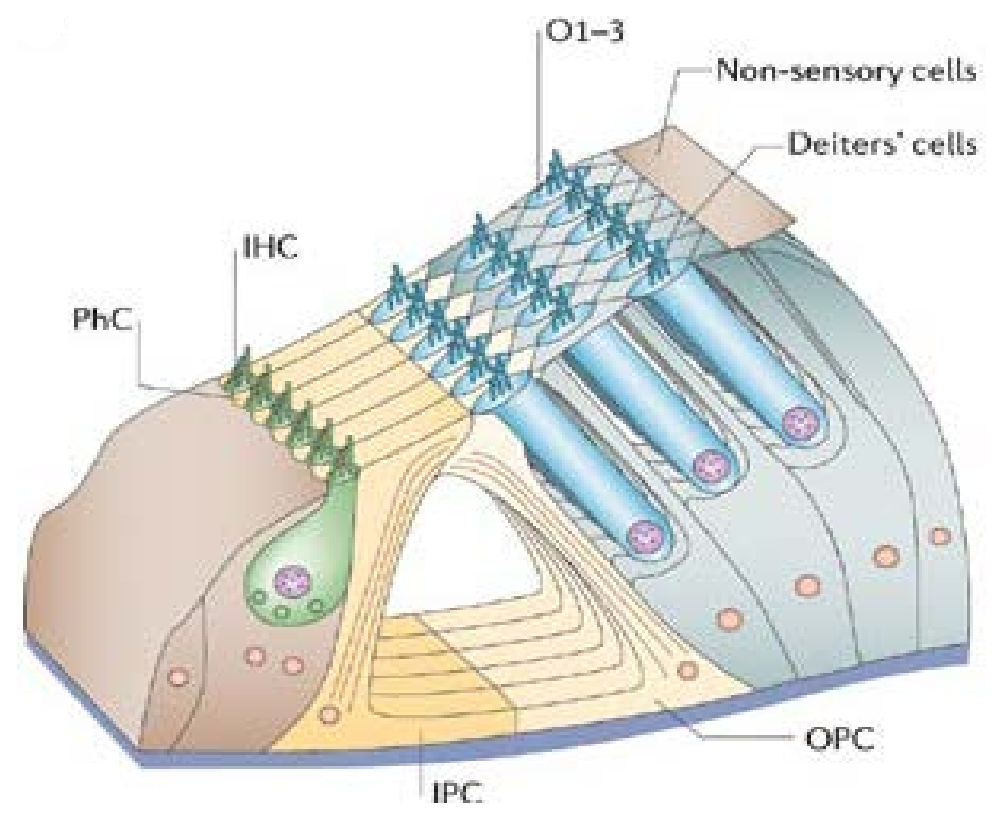

Figure 10 - Cochlear Hair Cells: Inner (green) and Outer (blue) hair cells are shown in contrast. The ovoid shape of the IHC shows that nature has emphasized its neurotransmitter function while the cylindrical shape of the OHCs (with the nucleus at the basal end of the cell - purple) allows them to perform their roles as tuners by amplifying and enhancing sound vibrations. IHCs are separated from each other by inner phalangeal supporting cells $(\mathrm{PhC})$ while OHCs instead rely on Deiters' supporting cells. Inner and Outer Pillar Cells (IPC, OPC) are also distinguished.

This function is to be emphasized since $95 \%$ of the afferent (to the brain) nerve fibres in the auditory nerve system are connect to IHC pathways, highlighting the critical function of IHCs as the 'messengers' of the hearing system. Indeed our "sense of hearing depends on this fast, finely graded neurotransmission at the ribbon synapses connecting (inner) hair cells to afferent nerve fibers." Figure 10 from [38] shows the structures and contents of both IHC and OHC cells.

The process of amplifying and tuning of sounds by OHCs has been described as 'stimulated acoustic emission' as early as 1978 by D. T. Kemp [39], indicating the requirement of an input to trigger the system. As the $\mathrm{OHC}$ resonates at the frequency of this input, it stretches and contracts length-wise to amplify the signal. Three rows of OHCs line up side by side all along the basilar membrane of the organ of Corti, feeding mechanical power into the organ of Corti by way of amplifying sound. Incoming vibrations are detected by the bundle of hair-like stereocilia 
fibres on their apical surfaces. Once detected, the flexoelectric properties of the OHC cell wall coupled with viscoelastic interfacial surroundings allow the resonant OHCs (determined by length) to vibrate along with the frequency of input vibrations, amplifying and modulating the resonance in the process. The average lengths of the $\mathrm{OHCs}$ vary along the cochlea, ranging from shortest at the basal end of the cochlea - high frequency detection, and longest at the apex of the cochlea - low frequency detection, as cell lengths range from $20 \mu \mathrm{m}$ up to $80 \mu \mathrm{m}$.

In Figure 11 from [6], a longitudinal $\mathrm{OHC}$ cross section shows a stereocilia bundle in a "W" pattern formed by rows of three to four cells that curve together. The outermost stereocilia are longer than their inner counterparts and are lodged in the overhanging tectorial membrane. Between individual stereocilia are links that connect the stereocilia. Two sets of links exist: lateral links and tip links. While tip links are discussed later, lateral links form between stereocilia of the same as well as different rows, fastening the entire structure.

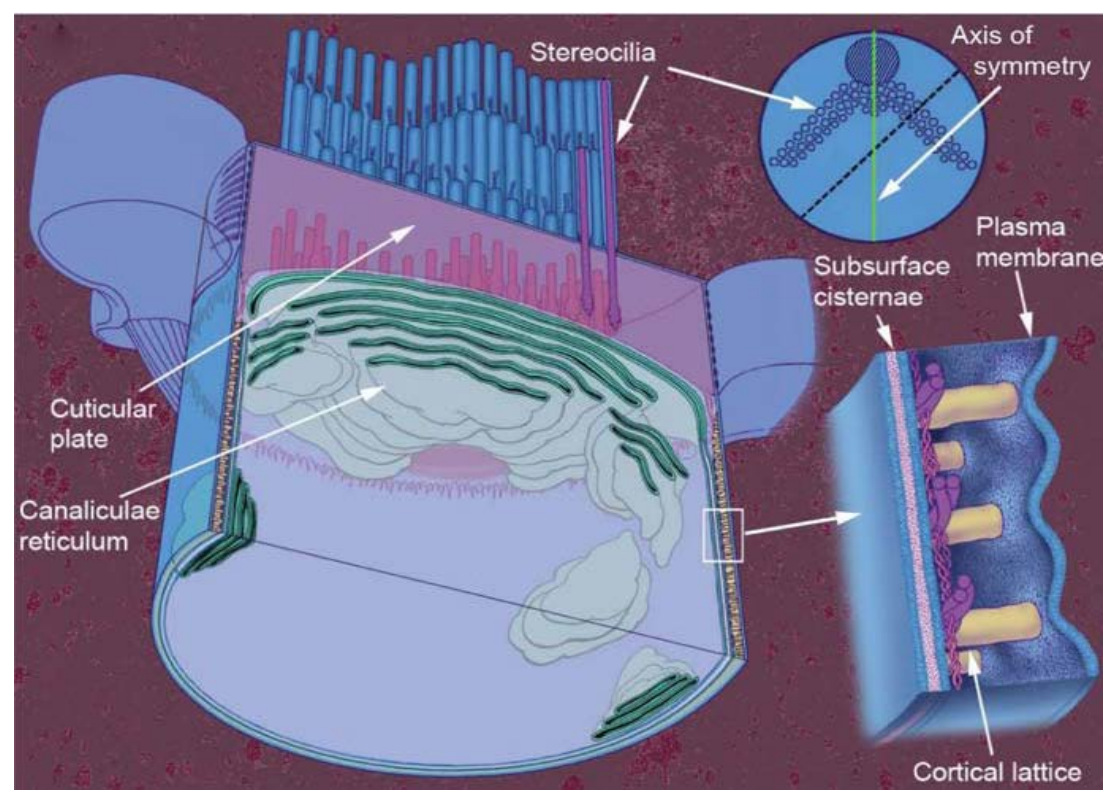

Figure 11 - Apical Surface of OHC: Shown above through the cuticular plate of the OHC are the stereocilia, forming a bundle. The cytoskeletal layer, called the subsurface cisternae, is held together by a pillar-like lattice. 


\subsubsection{OHC Structure and Function}

Structurally, the $\mathrm{OHC}$ can be divided into three main areas: the apical pole, the basal pole and the lateral wall of the cell. The apical pole (top of the $\mathrm{OHC}$ ) consists of a stereocilia bundle and a cuticular plate (CP) above which the bundle is attached. The OHC's CP is thick enough such that the stereocilia rootlets do not penetrate the layer. The tips of the tallest stereocilia reach up into the tectorial membrane where they are securely attached. The basal pole (bottom of the $\mathrm{OHC}$ ) contains the cell nucleus and nerve synapses. While very few afferent synapses are found in the OHC there are many efferent synapses, suggesting OHCs also behave as sensors, picking up tuning signals from the brain and determinately altering their amplification factors. This functionality allows the brain to control the 'gain' of the OHC, very useful for instance as a response to excessive volume and other stresses. Finally, the vehicle of amplification in the $\mathrm{OHC}$ is its flexoelectric lateral wall, where the plasma membrane (PM) and subsurface cisternae (SSC) are found.

The third part of the $\mathrm{OHC}$ is the most important in the electromotility of the cell. The lateral wall of the $\mathrm{OHC}$ is a three layer structure, as seen in Figure 12 from [40]. The outer most layer is the plasma membrane, which may contain less cholesterol than other parts of the OHC.

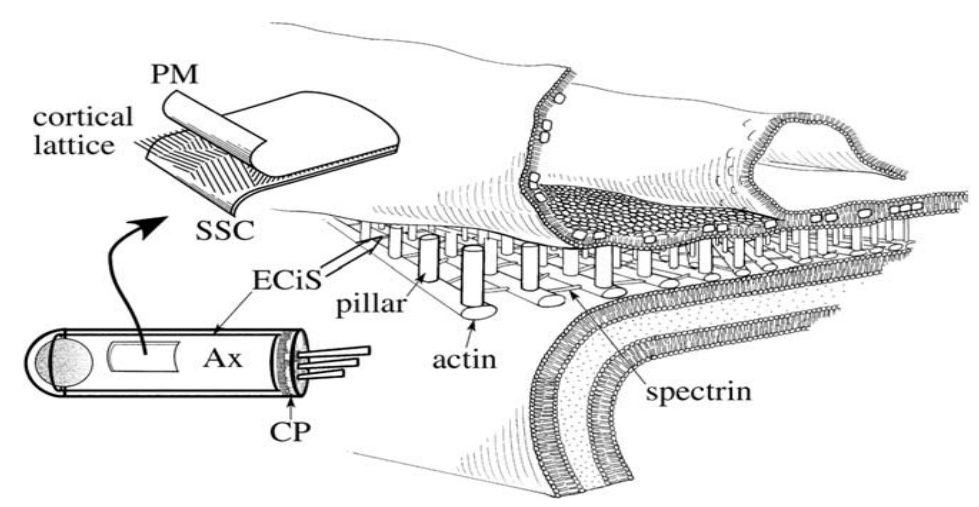

Figure 12 - The plasma membrane (PM) the cortical lattice and the subsurface cistern (SSC) 
A membrane that contains less cholesterol is "softer" and more flexible. The inner most layer is an about $30 \mathrm{~nm}$ wide structure related to the endoplasmic reticulum called the subsurface cisterna (SSC). The SSC is actually suspected to be apparented to the sarcoplasmic reticulum in the muscle and be a container of $\mathrm{Ca}^{2+}$. The other layer between the plasma membrane and the SSC is an arrangement of macromolecules called the cortical lattice $(\mathrm{CL})$. This set of macromolecules is composed of actin filaments and spectrin molecules perpendicular to each other. The actin filaments are generally circumferential and the spectrin proteins are generally longitudinal. Pillars of unknown molecular composition are also present in the $\mathrm{CL}$ and are oriented radially to the cell. These pillars tether strongly the PM and make, with the help of the spectrin molecules, microvillosities all along the length of the OHC. There is a 40 to $50 \mathrm{~nm}$ space between the molecules of spectrin [41]. The plasma membrane of the lateral wall contains a few transmembrane proteins that are greatly expressed such as a modified anion exchanger AE2, a sugar transporter GLUT-5 and prestin [42]. Stretch-activated ion channels were also found in the plasma membrane of the lateral wall of $\mathrm{OHC}$ even though the nature of these channels is not known.

The Inner Hair Cells are the "true" sensitive cells. They are the one which transmit the information to the auditory nerve and, therefore, to the brain. The role of the OHC amplifies the information for the IHC. That is, the IHC would be insufficient to detect sound waves without the effect of the OHC. Furthermore, the "cochlear amplifier" refines the sensitivity and frequency selectivity of the mechanical vibrations of the cochlea. The fact that the cochlea is a liquid filled structure dampens the effect of a sound wave coming from the air [43]. This characteristic is suspected to be inherited from our animal ancestors that lived underwater. This 
amplification has permitted mammals to increase the range of frequencies perceived, especially in the high frequency domain. The evolution selection has favored the development of the $\mathrm{OHC}$, which are considered to be the "most exotically specialized hair cells" [44]. OHC amplify the signal by changing it's length and, therefore, enhancing the movement of the tectorial membrane. The change in length is called the electromotility and is ATP-independent. This amplification, called the otoacoustic emissions (OAE), can be detected by clinical methods. Audiologists detect malfunctions in OHC by measuring the OAE. It can be considered as a sound coming from the cochlea in response to an external stimulus. If the patient doesn't "return" any $\mathrm{OAE}$, it means that his $\mathrm{OHC}$ are not functioning correctly, more precisely they do not exhibit electromotility, thus not amplifying the sound for the IHC. The patient, therefore, has a hearing disorder.

When a sound is detected by the tympanic membrane, it will oscillate the ossicles at a given frequency, that will themselves oscillate the oval window. The changes in pressure will deform the basilar membrane that will have a maximal amplitude at a given location known as the characteristic frequency (CF) because of the different mechanical properties at each locations of the cochlea. The organ of Corti being seated on the basilar membrane, the OHC located at the CF will move with the transmission of the sound wave. As stated above, the tips of the tallest stereocilia are embedded in the tectorial membrane and all the stereocilia of a same cell are connected by links. When the basilar membrane is deformed, it pushes on the Dieter's cells, which make the $\mathrm{OHC}$ move. Since the tectorial membrane is practically floating in the endolymph, the movement of the $\mathrm{OHC}$ will create a shear movement between the tectorial membrane and the $\mathrm{OHC}$ and make the tallest stereocilia tip on a side, pulling on the entire 
bundle attached. The pressure imposed by these tip links will open some gate channels on the stereocilia and there will be an entrance of $\mathrm{Ca}^{2+}$ ions in the cell. The $\mathrm{OHC}$ will then be depolarized and shortened while it's membrane will get stiffer. The entrance of $\mathrm{Ca}^{2+}$ will also make the channel lower on the stereocilia by the use of actin filaments and motor proteins like myosin, making the pressure imposed on the tip links drop. The gate will then close and hyperpolarization will be possible, see Figure $\mathbf{1 3}$ from [45]. Hyperpolarization will have the opposite effect on the cell, elongating the cell and making reducing membrane stiffness. It is the vibrational operation back and forth from hyperpolarization to depolarization that allows the $\mathrm{OHC}$ to be studied as a bending membrane along its lateral wall. This effect is called electromotility, the motion of a cell's body in response to externally or internally produced electric fields.
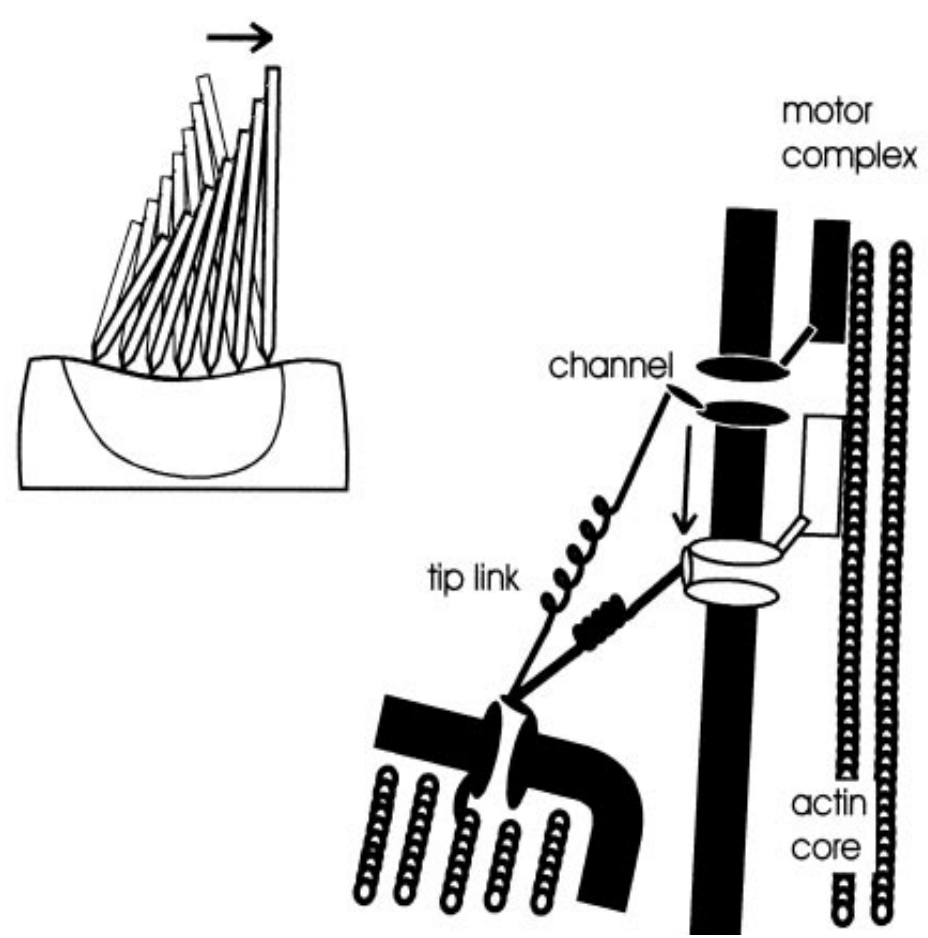

Figure 13 - Scheme of a part of two cilias: When the stereocilia bundle tips to a side, the tip link will open a gate channel. The entrance of $\mathrm{Ca}^{2+}$ will activate a motor complex that will use an actin filament to diminish the pressure imposed on the tip link, closing the gate channel. 


\subsubsection{OHC Electromotility}

The electric potential has an effect on the curvature of the plasma membrane of the cell by a mechanism called flexoelectricity. Flexoelectricity is a physical property of liquid crystals similar to piezoelectricity in solid crystals [17]. Liquid crystals are liquids that have some sort crystalline organisation. Plasma membranes are therefore considered as liquid crystals since their molecules have a constant orientation. In the case of $\mathrm{OHC}$, the converse flexoelectric effect is considered to play an important role. The electric field potential imposed by the action of the molecular motor prestin will make the membrane bend. Since the lateral wall of the $\mathrm{OHC}$ is formed of many microvillosities, the sum of all the changes in the bending of each of these microvillosities will shorten the cells in the axial direction (Figure 14 from [6]). The circumferential direction remains the same because of the array of actin filaments that prevents the deformation of the cell in the circumferential direction. The cell will then shorten when it will be depolarized, which is what is observed in experiments. The spectrin molecules located between the microvillosities will impose some resistance to the length changes in the cell.

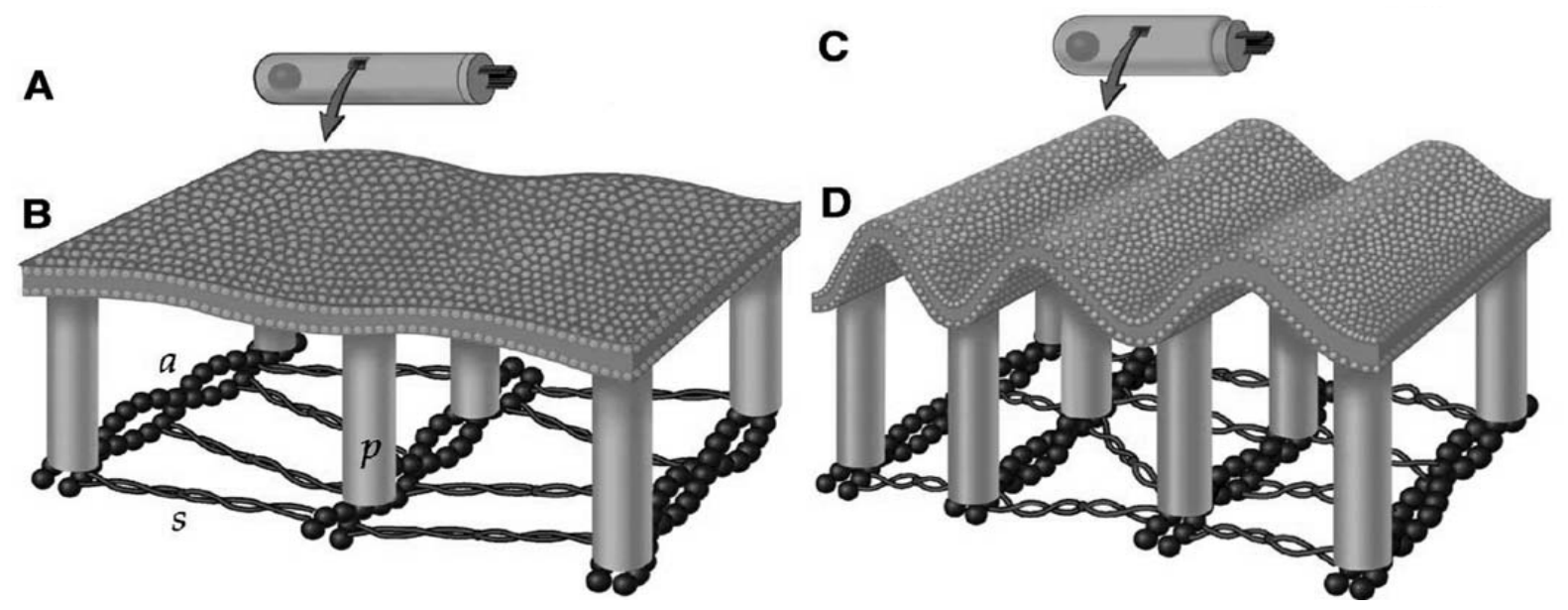

Figure 14 - Illustration of $\mathrm{OHC}$ bending. $[\mathrm{A}]$ and $[\mathrm{B}]$ show an $\mathrm{OHC}$ hyperpolarized. The cell is longer and the curvature of the plasma membrane of is less than depolarized $\mathrm{OHC}[\mathrm{C}, \mathrm{D}]$. 


\subsection{Modeling a Bending Membrane in Viscoelastic Media}

In order to completely account for surrounding effects, dynamic models for bending membranes must account for viscoelasticity. Since many biological systems operate in a viscoelastic environment, one must consider 'elasticity' as well as the 'viscosity' of materials in the system. Petrov [2-6] is widely recognized as a pioneer in the field of nematic liquid crystal flexoelectricity and his formulations for membrane deformations including curvature remain in use and act as important references. After briefly reviewing some groundwork in the field we explain linear material viscoelasticity before describing some simple models for viscoelastic systems. We finally discuss the relation of viscoelasticity to soft bio-matter such as the OHC as well as its particular resonant behaviour, hypothesized to counteract viscosity by amplifying incoming high frequency vibrations to support our hearing system.

In this thesis we liken the function of the $\mathrm{OHC}$ to a bending membrane between two viscoelastic fluids. This viscoelastic-electromechanical coupling represents two thirds of the main strategy of our research, where the final segment involves the membrane shape equation derived earlier from Helfrich equations. To avoid redundancy these details are omitted from the thesis as considerable detail for this derivation is provided in the literature $[16,18]$. Whereas elasticity is usually the result of bond stretching along crystallographic planes in an ordered solid, viscosity is the result of the diffusion of atoms or molecules inside an amorphous material. Viscoelasticity is defined as the property of materials that exhibit both viscous and elastic characteristics under deformation. Purely viscous materials resist shear flow and strain linearly with time when a stress is applied, purely elastic materials strain instantaneously when stretched and just as quickly return to their original state once the stress is removed, while 
viscoelastic materials have elements of both of these properties and as such exhibit time dependent strain. Another way to understand viscous and elastic effects is to consider a material with "infinite memory" and for which stress and strain are related elastically, as springs, and viscidly, as dashpots:

$$
\begin{gathered}
\sigma=E \varepsilon \\
\sigma=\eta \frac{d \varepsilon}{d t}
\end{gathered}
$$

Where $\sigma$ is stress and $\varepsilon$ is strain, $E$ is the material elastic modulus and $\eta$ the material viscosity. Simple spring-and-dashpot models are used to model viscoelastic materials, the simplest of which are Maxwell (left) and Kelvin-Voigt (right) models, shown respectively in Figure 15:

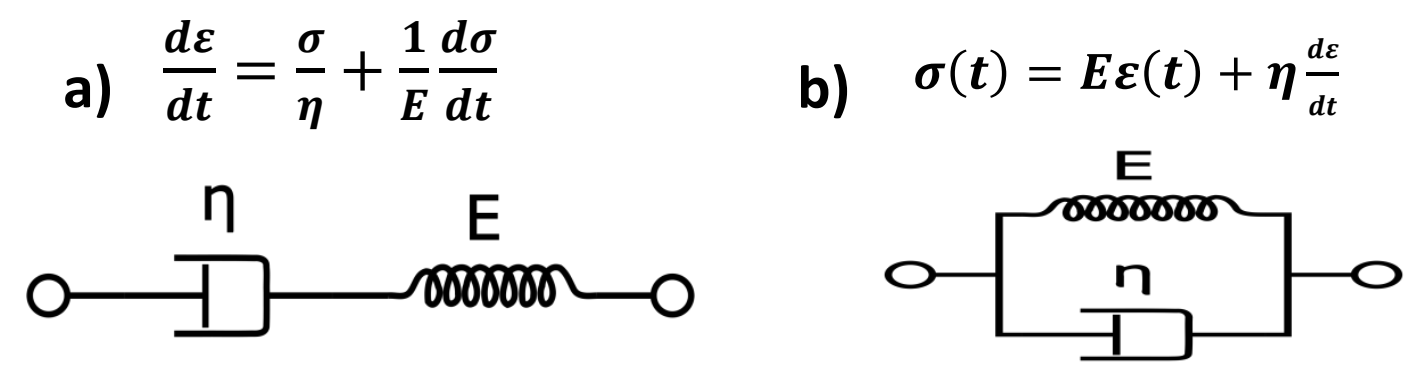

Figure 15 - (a) Maxwell viscoelastic model and (b) Kelvin-Voigt viscoelastic model.

Though useful for a variety of applications the level of accuracy of both these simple models is generally inadequate for dynamic modeling in biological materials. Also, these models aim to identify stress-strain relations whereas in our model we analogously relate curvature to electric field. In our integrated model we generalize and make use of the nonlinear Upper Convected Maxwell Model which allows for robust modeling of material surroundings and is generally acceptable for biological modeling. For the case of small deformations the nonlinearities disappear and the model becomes an ordinary Maxwell model. 


\subsection{Research and Methodology}

In undertaking this project the bulk of the labor has come through innovative mathematical derivations and understanding subsequent results. Since the inspiration of our research came largely from the biophysical phenomenon of the $\mathrm{OHC}$, our results were measured and reflected off literature to ensure accurate representation. Beyond the background provided in this chapter, we proceed in Chapter 3 to connect the five key aspects of our thesis: (i) OHC mechanotransduction, (ii) Flexoelectricity in biological membranes, (iii) Liquid crystal model of flexoelectric membranes, (iv) Viscoelastic fluids and (v) Membrane elasticity. These five topics form the basic input into the model we present, solve, analyze, and validate in Chapter 3 . A flow-chart in Figure 16 below breaks down the methodology followed in the chapter.

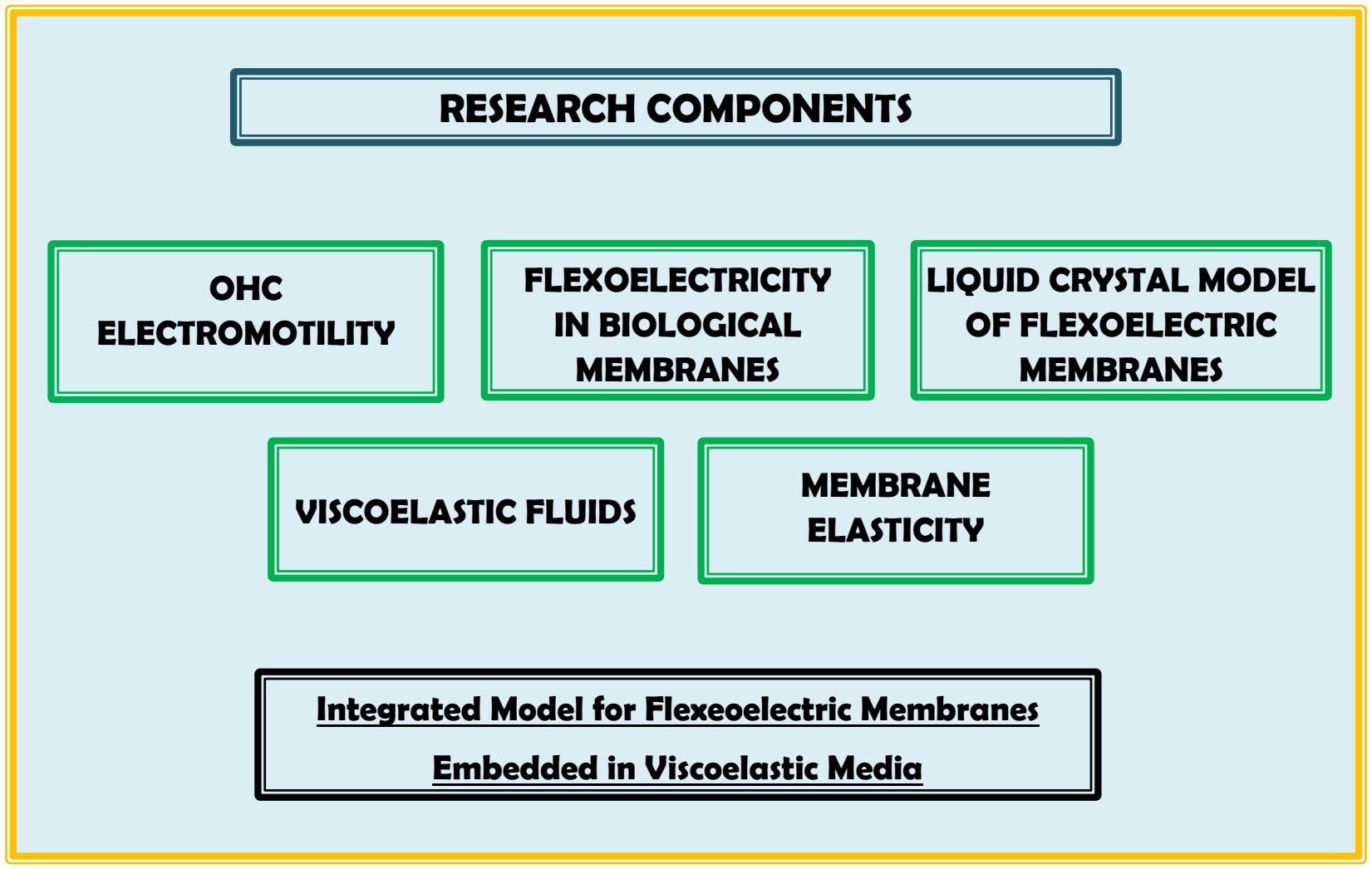

Figure 16 - Results are understood through 5 key components in our proposed model shown in green. 


\section{Chapter 3}

\section{Flexoelectric-Viscoelastic Model}

A rigorous procedure was required to develop our $2^{\text {nd }}$ order ODE membrane bending flexoelectric model from first principles, so in this Chapter the body of pertinent derivations of our mathematical model is presented as well as results and analysis. First we summarize the model background and delve into mapping and contextualizing our analytical equations and corresponding coefficients. As an integrated model of $2^{\text {nd }}$ order flexoelectric behaviour we further describe properties such as mechanical response, energy and power considerations after which we analyze the viscous, elastic and inertial forces in our system. In closing, we tabulate and categorize our results to provide reference and facilitate the expansion of future work in this field.

\subsection{Actuator Model for Flexoelectric Membranes}

To avoid repetition of lengthy derivations the reader is referred to the previous works for flexoelectric membrane in an inviscid and viscous cases respectively $[16,18]$. The physical setup and geometry of the flexoelectric membrane tethered to a capillary tube containing two viscoelastic fluids is defined in Figure 17. A capillary tube of radius a contains an edge-fixed flexoelectric membrane located at $\mathrm{z}=0$. Above and below the membrane there are two viscoelastic fluids with column heights $\mathrm{z}=\mathrm{L}$, viscosities $\left\{\eta_{\mathrm{b}}, \eta_{\mathrm{t}}\right\}$ and relaxation times $\left\{\lambda_{\mathrm{b}}, \lambda_{\mathrm{t}}\right\}$ respectively. The pressure at the top of the upper layer and at the bottom of the lower layers are equal to a constant $\mathrm{P}_{0}$, by imposing a fluctuating $\mathbf{E}$ field the membrane oscillates and 
displaces the upper and lower incompressible viscoelastic fluids ; we emphasize that the Poiseuille flow is only generated by the flexoelectric effect of the membrane caused by the imposed $\mathbf{E}(\mathrm{t})$ field.. The membrane deformation is described by a spherical dome of height $\mathrm{h}$ and radius $\mathrm{R}$.

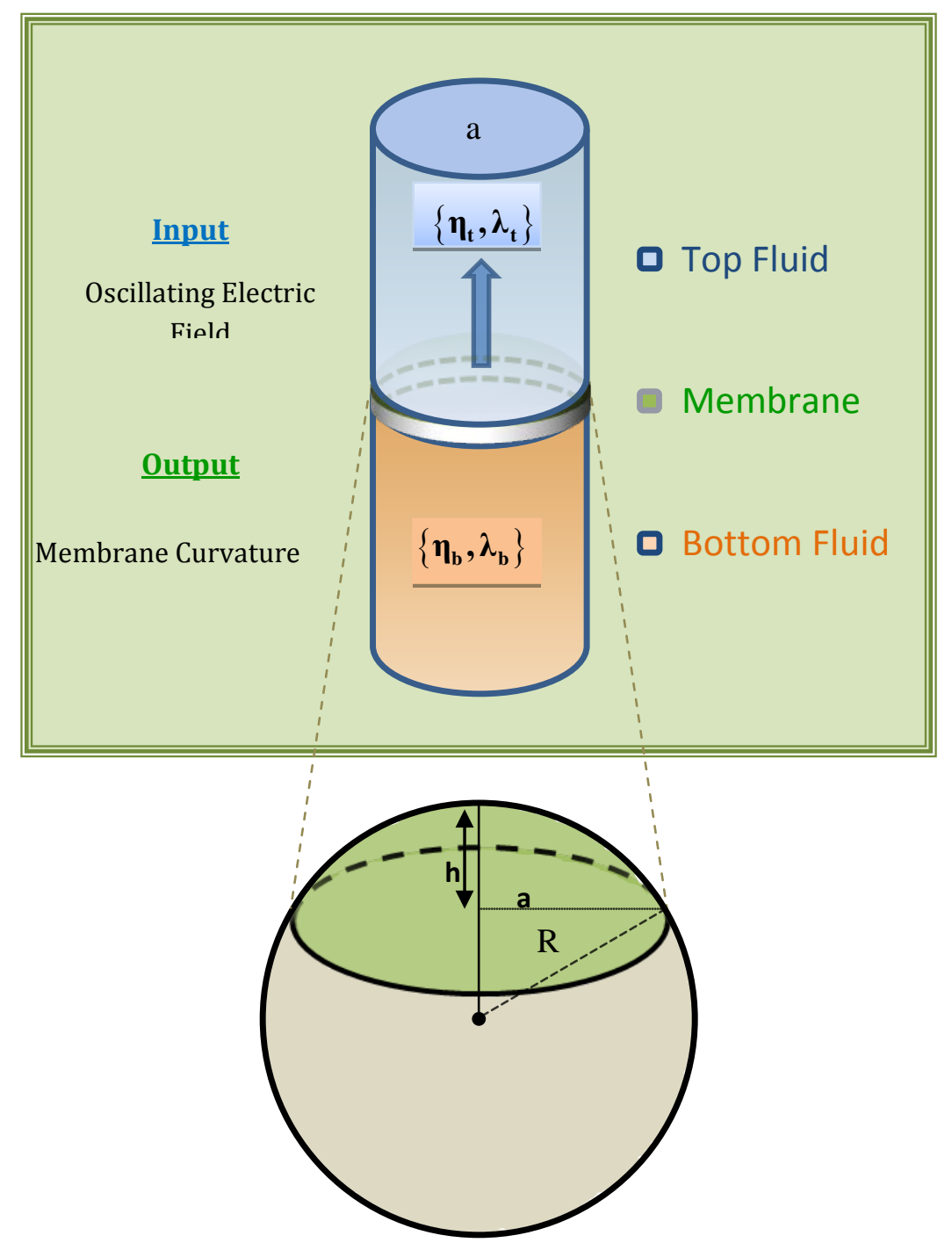

Figure 17 - Schematic of the geometry and operation of flexoelectric mechanics, defined in Figure 2, in a capillary geometry of radius $\mathbf{a}$. he input $\mathbf{E}$ field distorts the initially flat circular membrane into a spherical cap of radius $\mathrm{R}$ and height $\mathrm{h}$. The flexoelectric actuation creates a capillary viscoelastic flow in the contacting top $(t)$ and bottom (b) fluids of viscosity $\left\{\eta_{t}, \eta_{b}\right\}$ and retardation time $\left\{\lambda_{t}, \lambda_{b}\right\}$. 
The shape equation that describes the average curvature $\mathrm{H}(\mathrm{t})$ dynamics of the membrane is found by formulating the normal stress balance equation across the oscillating flexoelectric membrane $[16,18]$ :

$$
\underbrace{\mathrm{C}_{\mathrm{f}} \mathfrak{I} \mathbf{E}(\mathrm{t})}_{\begin{array}{c}
\text { input flexoelectric } \\
\text { driving force }
\end{array}}=\underbrace{\left|\mathbf{k k}: \Delta \mathbf{T}_{\mathrm{b}}\right|(\mathrm{t})}_{\begin{array}{c}
\text { bulk viccoelastic } \\
\text { fluids' stress jump }
\end{array}}+\underbrace{\left(2 \gamma_{\mathrm{o}}+\left(2 \mathrm{k}_{\mathrm{c}}+\overline{\mathrm{k}}_{\mathrm{c}}\right) \mathfrak{I}\right) \mathrm{H}(\mathrm{t})}_{\text {restoring membrane elastic force }}
$$

where the geometric factor $1 / \mathfrak{I}=\mathrm{a}^{2} / 8$ indicates the characteristic deformation area associated with the spherical cup shown in Figure 3. The shape equation (9) is a balance between membrane flexoelectric force, bulk viscoelastic liquid stress jump across the membrane and restoring membrane elastic force. The oscillating flexoelectric force $\mathbf{F}_{\mathrm{E}}(\mathrm{t})$

$\mathbf{F}_{\mathrm{E}}(\mathrm{t})=\frac{8 \mathrm{c}_{\mathrm{f}} \mathbf{E}(\mathrm{t})}{\mathrm{a}^{2}}$

is proportional to the externally imposed $\mathbf{E}(\mathrm{t})$ field and the flexoelectric coefficient $\mathrm{C}_{\mathrm{f}}$ indicates the converse effect, through which a $\mathbf{E}(\mathrm{t})$ field creates the membrane vertical displacement. As the membrane fluctuates, the contacting viscoelastic fluids dissipate and store energy through the oscillating upward and downward capillary flow. The net vertical bulk force at the membrane $\left|\mathbf{k k}: \Delta \mathbf{T}_{\mathrm{b}}\right|(\mathrm{t})$ contains both viscous and elastic contributions and is computed from the oscillatory viscoelastic capillary flow over a tube of total length $\mathrm{z}=2 \mathrm{~L}$ (see eqn.(B-14) in Appendix B) :

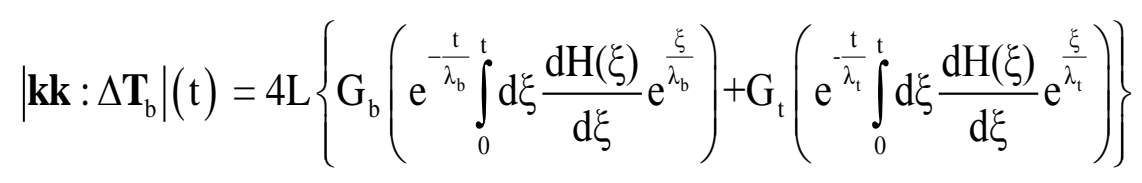


where $\mathrm{G}_{\mathrm{i}}=\eta_{\mathrm{i}} / \lambda_{\mathrm{i}} ; \mathrm{i}=\mathrm{t}, \mathrm{b}$ are respectively the elastic modulus, viscosity and retardation time of the top (t) and bottom (b) viscoelastic liquids. The stress jump is linear with $\mathrm{L}$. The integrand contains the curvature speed $\mathrm{dH}(\xi) / \mathrm{d} \xi$ which is proportional to the volumetric flow rate $\mathrm{Q}(\mathrm{t})$ by (see Appendix B) :

$\frac{\mathrm{dH}(\xi)}{\mathrm{d} \xi}=-\frac{2 \mathrm{Q}(\xi)}{\pi \mathrm{a}^{4}}$

and as $\mathrm{H}(\mathrm{t})$ oscillates so does $\mathrm{Q}(\mathrm{t})$. The membrane elasticity gives rise to a restoring force proportional to the membrane average curvature $H(t)=-R^{-1}(t)$ :

$\mathbf{F}_{\text {menbrane }}(\mathrm{t})=2\left(\gamma_{\mathrm{o}}+\left(2 \mathrm{k}_{\mathrm{c}}+\overline{\mathrm{k}}_{\mathrm{c}}\right) \frac{\mathfrak{I}}{2}\right) \mathrm{H}(\mathrm{t})$

where $\gamma_{\mathrm{o}}+\left(2 \mathrm{k}_{\mathrm{c}}+\overline{\mathrm{k}}_{\mathrm{c}}\right) \mathfrak{I} / 2$ is the effective membrane tension that includes the membrane tension $\gamma_{0}$, bending $\mathrm{k}_{\mathrm{c}}$ and torsion $\overline{\mathrm{k}}_{\mathrm{c}}[16,18]$ from edge effects. Assuming a small amplitude harmonic field $\mathbf{E}(\mathrm{t})$, capillary Poiseuille flow in the contacting viscoelastic fluids, and spherical membrane distortions, the following second order linear curvature dynamics is obtained (see Appendix B) :

$\underbrace{\mathrm{b}_{2} \ddot{\mathrm{H}}(\mathrm{t})}_{\text {Inertia }}+\underbrace{\mathrm{b}_{1} \dot{\mathrm{H}}(\mathrm{t})}_{\text {Viscous-Bulk }}+\underbrace{\mathrm{b}_{0} \mathrm{H}(\mathrm{t})}_{\text {Elastic-Membrane }}=\underbrace{\mathrm{a}_{2} \ddot{\mathrm{E}}(\mathrm{t})+\mathrm{a}_{1} \dot{\mathrm{E}}(\mathrm{t})+\mathrm{a}_{0} \mathrm{E}(\mathrm{t})}_{\text {Flexoelectric Force }}$

The right hand side is the flexoelectric input which is of the same order as the curvature output. The curvature response is a balance between bulk inertial $b_{2} \ddot{H}(t)$, bulk viscous 
$b_{1} \dot{H}(t)$, and membrane elastic forces $b_{0} H(t)$. The above equation satisfies the two initial conditions for the curvature and the time derivative of the curvature:

$\mathrm{H}(0)=\mathrm{H}_{0} ; \dot{\mathrm{H}}(0)=\mathrm{H}_{1}$

The coefficients and material functions in equation (14) are given by:

input: $\left\{\begin{array}{l}\mathrm{a}_{\mathrm{o}}=\frac{\mathrm{c}_{\mathrm{f}} \mathfrak{I}}{4 \mathrm{~L}} \\ \mathrm{a}_{1}=\mathrm{a}_{\mathrm{o}} \Sigma_{\lambda}, \Sigma_{\lambda}=\left(\lambda_{\mathrm{t}}+\lambda_{\mathrm{b}}\right) \\ \mathrm{a}_{2}=\mathrm{a}_{\mathrm{o}} \Pi_{\lambda}, \Pi_{\lambda}=\lambda_{\mathrm{t}} \lambda_{\mathrm{b}} \\ \text { output : }\left\{\begin{array}{l}\mathrm{b}_{1}=\Sigma_{\mathrm{G} \lambda}+\mathrm{M} \Sigma_{\lambda}, \Sigma_{\mathrm{G} \lambda}=\eta_{\mathrm{b}}+\eta_{\mathrm{t}} \\ \mathrm{b}_{0}=\frac{1}{2}\left(\frac{\gamma_{\mathrm{o}}}{\mathrm{L}}+\frac{\left(\mathrm{k}_{\mathrm{c}}+\overline{\mathrm{k}}_{\mathrm{c}} / 2\right) \mathfrak{I}}{\mathrm{L}}\right) \equiv \mathrm{M} \\ \mathrm{b}_{2}=\mathrm{X}_{\eta \lambda}+\mathrm{M} \Pi_{\lambda}=\left(\Sigma_{\mathrm{G}}+\mathrm{M}\right) \Pi_{\lambda}, \Sigma_{\mathrm{G}}=\mathrm{G}_{\mathrm{b}}+\mathrm{G}_{\mathrm{t}}, \mathrm{X}_{\eta \lambda}=\eta_{\mathrm{b}} \lambda_{\mathrm{t}}+\eta_{\mathrm{t}} \lambda_{\mathrm{b}}\end{array}\right.\end{array}\right.$

The membranodynamic model given by equations (14-16a-f) contains seven primitive materials properties: (i) viscoelastic properties $\left\{\eta_{b}, \lambda_{b}, \eta_{t}, \lambda_{t}\right\}$; (ii) membrane elasticity properties: $\left\{\gamma_{\mathrm{o}}, \mathrm{k}_{\mathrm{c}}, \overline{\mathrm{k}}_{\mathrm{c}}\right\}$; (iii) geometry of the pipe and the membrane $\{\mathrm{a}, \mathrm{L} ; \mathfrak{I}\}$; and (iv) flexoelectric force: $\left\{c_{f}, E_{0}\right\}$. The elastic moduli of the viscoelastic fluids are: $G_{i}=\eta_{i} / \lambda_{i} ; i=t, b$. The two products properties a $X_{\eta \lambda}$ and $\Pi_{\lambda}$. The membrane stiffness $M$ appears in $\left\{b_{0}, b_{1}\right\}$ 
and $b_{2}$. All these parameters can be estimated from rheological and membrane experiments in steady and unsteady state [46-52]. Equations (14-16a-f) describe an electro-viscoelastic system with three phases, two viscoelastic liquids and one elastic membrane. The case of purely viscous bulk phases was treated in [16]. We note that the mechanical stress/strain analogue of our viscoelastic/flexoelectric membrane under capillary confinement is the four element Burger's constitutive equation for viscoelastic solids [53-62]. Below, we use $b_{0} \equiv M$ without ambiguity to either emphasize the dynamical system $\left(b_{0}\right)$ or the material aspects $(M)$.

\subsubsection{Three Dimensional Material Phase Space}

Figure 18 shows the 3D material parametric space of the bulk viscoelastic/flexoelectric membrane system defined by the vector $\mathbf{S}$ :

$\mathbf{S}(\mathrm{M})=\left(\mathrm{M}, \mathrm{b}_{1}(\mathrm{M}), \mathrm{b}_{2}(\mathrm{M})\right)=\left(\mathrm{M}, \Sigma_{\eta}+\mathrm{M} \Sigma_{\lambda}, \mathrm{X}_{\eta \lambda}+\mathrm{M} \Pi_{\lambda}\right)$

The coordinates indicate the strength of the three mechanisms: inertia, viscosity, and elasticity defined in eqns.(16e-f).

The projections of $S$ onto the three planes define three important operating lines, shown in Figure 19. (a) Viscous/elastic plane: in the elasto-viscous plane the operating line $b_{1}(M)$ is:

$\mathrm{b}_{1}(\mathrm{M})=\Sigma_{\eta}+\Sigma_{\lambda} \mathrm{M}$

This equation represents a line with a positive slope: $\Sigma_{\lambda}$ (retardation) and abscise: $\Sigma_{\eta}$ (viscosity) . The viscous coefficient $\mathrm{b}_{1}(\mathrm{M})$ can be increased by both the bulk viscosity $\Sigma_{\eta}$ and the elasticity $\Sigma_{\lambda} M$. (b) Elasto/inertial plane: the operating line $b_{2}(M)$ is: 
$\mathrm{b}_{2}(\mathrm{M})=\mathrm{X}_{\eta \lambda}+\Pi_{\lambda} \mathrm{M}$

with slope $\Pi_{\lambda}$ and abscise $X_{n \lambda}$. The inertia coefficient can be increased by both viscoelastic coupling $X_{\eta \lambda}=\eta_{b} \lambda_{t}+\eta_{t} \lambda_{b}$ and elastic coupling $\Pi_{\lambda} M=\lambda_{t} \lambda_{b} M$. (c) Visco/inertial plane: The operating line in the $M=0$ plane is the relation between inertial $b_{2}$ and viscous $b_{1}$ coefficients:

$\mathrm{b}_{2}(\mathrm{M})=\Sigma_{\lambda} \mathrm{b}_{1}(\mathrm{M})-\mathrm{X}_{\eta \lambda}$

More retardation $\Sigma_{\lambda}$ enhances inertia the coefficient $b_{2}$ for a given viscous coefficient $b_{1}$.

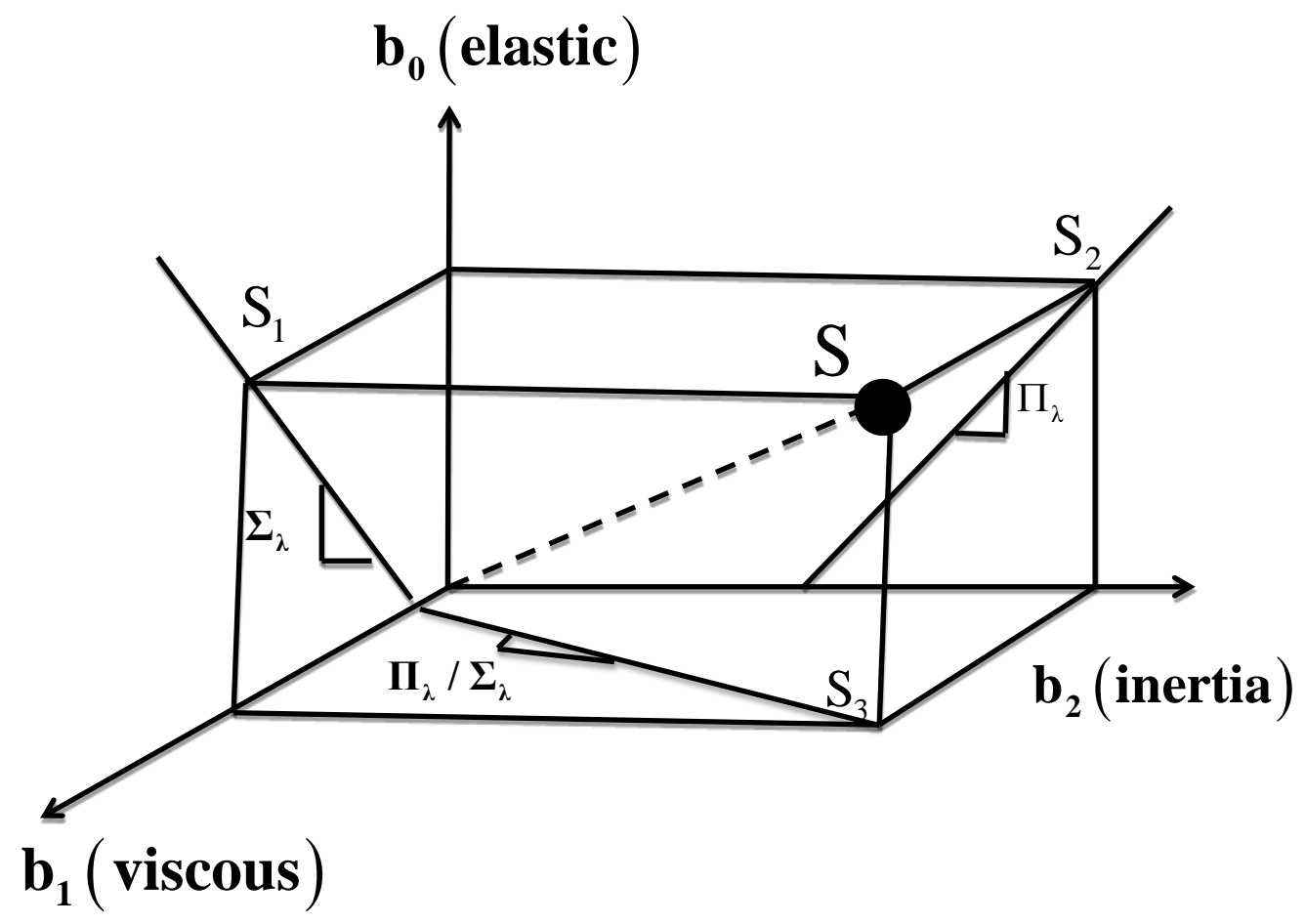

Figure 18. The flexoelectric membrane-viscoelastic fluid actuation system is defined by $S$ (dashed line) whose coordinates $\mathbf{S}=\left(\mathrm{S}_{1}, \mathrm{~S}_{2}, \mathrm{~S}_{3}\right)$ are membrane elasticity $\mathrm{b}_{0}=\mathrm{M}$, the viscosity function of the bulk viscoelastic fluids $b_{1}(M)$, and the inertia function of the bulk viscoelastic fluids $b_{2}(M)$ respectively. 


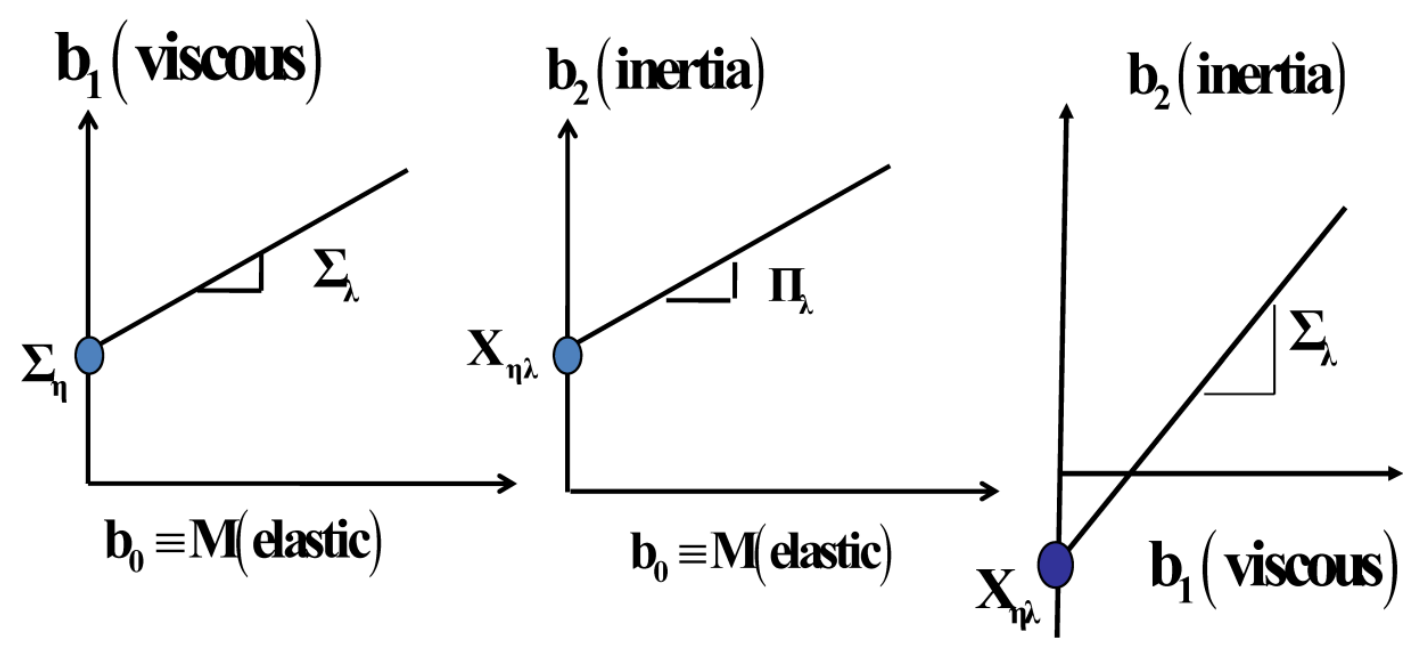

Figure 19. (Left) Schematic of material properties in the viscous/elastic plane (see eqn.(18)). (Right) Schematic of material properties in the elasto/inertial plane (see eqn.(19)). Operating line in the $M=0$ plane is the relation between inertial $b_{2}$ and viscous $b_{1}$ coefficients: $b_{2}=-X_{\eta \lambda}+\Sigma_{\lambda} b_{1}$ (see eqn. (20)).

\subsection{Dimensionless Model, Numbers and Material Functions}

Using routine non-dimensionalization, (see Appendix C) we obtain the dimensionless curvature dynamic equation - with initial conditions $\overline{\mathrm{H}}(0)=\overline{\mathrm{H}}_{0} ; \dot{\overline{\mathrm{H}}}(0)=\overline{\mathrm{H}}_{1}$ :

$\mathrm{b}_{2}^{*}\left(\mathrm{k}, \bar{\lambda}_{\mathrm{t}} \bar{\lambda}_{\mathrm{b}}\right) \ddot{\overline{\mathrm{H}}}(\overline{\mathrm{t}})+\mathrm{b}_{1}^{*}\left(\mathrm{k}, \boldsymbol{\Sigma}_{\bar{\eta}}\right) \dot{\overline{\mathrm{H}}}(\overline{\mathrm{t}})+\overline{\mathrm{H}}(\overline{\mathrm{t}})=\mathrm{kb}_{2}^{*}\left(\mathrm{k}, \bar{\lambda}_{\mathrm{t}} \bar{\lambda}_{\mathrm{b}}\right) \ddot{\overline{\mathrm{E}}}(\overline{\mathrm{t}})+\dot{\overline{\mathrm{E}}}(\overline{\mathrm{t}})+\overline{\mathrm{E}}(\overline{\mathrm{t}})(21)$

The differential equation given in (21a) contains three dimensionless groups $\left\{\bar{\lambda}_{\mathrm{t}} \bar{\lambda}_{\mathrm{b}}, \Sigma_{\bar{\eta}}, \mathrm{k}\right\}$, which are associated to different mechanisms in the system.

\section{a) Asymmetric phase number}

The first number $\left(\bar{\lambda}_{t} \bar{\lambda}_{b}\right)$ is associated to the product of the viscoelastic dimensionless times $\bar{\lambda}_{t}$ and $\bar{\lambda}_{b}$, linked by the following restriction: $\bar{\lambda}_{t}+\bar{\lambda}_{b}=1$. This numbers is related with the asymmetric phases in the system. An small value of $\bar{\lambda}_{t} \bar{\lambda}_{b}$ means that one of the viscoelastic liquid phase has a small dimensional Maxwell time and can be considered as a weak viscoelastic fluid. In contrast the other phase (second liquid) has a large Maxwell time and can be 
considered as a viscoelastic fluid. In contrast, when the two liquid phases are viscoelastic with the same numerical value of the Maxwell relation times, the value of the dimensionless number $\bar{\lambda}_{\mathrm{t}} \bar{\lambda}_{\mathrm{b}}$ is fixed and is equal to $\bar{\lambda}_{\mathrm{t}} \bar{\lambda}_{\mathrm{b}}=0.25$.

\section{b) Bulk-Viscous number}

The bulk viscous mechanism $\Sigma_{-\bar{\eta}}$, which is related with the total viscosity in the system (sum of the bottom and the top viscosities). The numerical value of this number is controlled by the product between the two dimensionless Maxwell time numbers $\bar{\lambda}_{t} \bar{\lambda}_{b}$, i.e., the value of the memory controls the value of the total bulk viscosity $\Sigma_{\bar{\eta}}=\Sigma_{\bar{\eta}}\left(\bar{\lambda}_{t} \bar{\lambda}_{\mathrm{b}}\right)$.

\section{c) Elastic ratio}

Finally, $\mathrm{k}=\frac{\overline{\mathrm{M}}}{\overline{\mathrm{M}}+1}$ is related to the elasticity in the membrane (solid) and the viscoelastic phases (liquid). This dimensionless number characterizes the state of the flexoelectric membrane. An small value of $\mathrm{k}$ means that the flexoelectric membrane is soft, whereas a value of $\mathrm{k}$ close to one, means that the flexoelectric membrane is a stiffness membrane respectively.

\section{d) Materials functions}

The dimensionless ordinary differential equation (21a) contains two dimensionless parametric material functions: $\left\{\mathrm{b}_{2}^{*}\left(\mathrm{k}, \bar{\lambda}_{\mathrm{t}} \bar{\lambda}_{\mathrm{b}}\right), \mathrm{b}_{1}^{*}\left(\mathrm{k}, \Sigma_{\bar{\eta}}\right)\right\}$ which are related to the asymmetric of the phases, bulk-viscous and elastic mechanisms through the characteristic dimensionless groups $\left\{\bar{\lambda}_{\mathrm{t}} \bar{\lambda}_{\mathrm{b}}, \boldsymbol{\Sigma}_{\bar{\eta}}, \mathrm{k}\right\}$ discussed earlier. These dimensional parametric functions describe a dimensionless material space $\mathrm{S}^{*}$ and are given by the following mathematical expressions:

(i) Dimensionless inertial function for the output $\overline{\mathrm{H}}(\overline{\mathrm{t}})$ 
$\mathrm{b}_{2}^{*}\left(\mathrm{k}, \bar{\lambda}_{\mathrm{t}} \bar{\lambda}_{\mathrm{b}}\right)=\frac{\bar{\lambda}_{\mathrm{t}} \bar{\lambda}_{\mathrm{b}}}{\mathrm{k}} \Leftrightarrow \mathrm{b}_{2}^{*}\left(\overline{\mathrm{M}}, \bar{\lambda}_{\mathrm{t}} \bar{\lambda}_{\mathrm{b}}\right)=\left(1+\frac{1}{\overline{\mathrm{M}}}\right) \bar{\lambda}_{\mathrm{t}} \bar{\lambda}_{\mathrm{b}}$

(ii) Dimensionless viscous function for the output $\overline{\mathrm{H}}(\overline{\mathrm{t}})$

$\mathrm{b}_{1}^{*}\left(\mathrm{k}, \Sigma_{\bar{\eta}}\right)=1+\left(\frac{1-\mathrm{k}}{\mathrm{k}}\right) \Sigma_{\bar{\eta}} \Leftrightarrow \mathrm{b}_{1}^{*}\left(\overline{\mathrm{M}}, \Sigma_{\bar{\eta}}\right)=1+\frac{\Sigma_{\bar{\eta}}}{\overline{\bar{M}}}$

In equations (22-23), we introduced the elasticity ratio $0<k<1$ :

$$
\mathrm{k}=\frac{1}{1+1 / \overline{\mathrm{M}}} \Leftrightarrow \overline{\mathrm{M}}=\frac{\mathrm{k}}{1-\mathrm{k}}
$$

Notice that the dimensionless functions $\left\{b_{2}^{*}\left(k, \bar{\lambda}_{t} \bar{\lambda}_{b}\right), b_{1}^{*}\left(k, \Sigma_{\bar{\eta}}\right)\right\}$ obey the following inequality:

$$
\underbrace{\mathrm{kb}=\bar{\lambda}_{\mathrm{t}}^{*} \bar{\lambda}_{\mathrm{b}}}_{\text {Memory }}<\underbrace{\mathrm{b}_{2}^{*}\left(\mathrm{k}, \bar{\lambda}_{\mathrm{t}} \bar{\lambda}_{\mathrm{b}}\right)}_{\text {Inertia }}<\underbrace{\mathrm{b}_{1}^{*}\left(\mathrm{k}, \boldsymbol{\Sigma}_{\bar{\eta}}\right)}_{\text {Bulk-Viscous }}
$$

It is important to note that these dimensionless numbers are bounded and satisfy the inequality given by equation 25 .

- The bounded asymmetric dimensionless number $\bar{\lambda}_{\mathrm{t}} \bar{\lambda}_{\mathrm{b}}$, i.e. $\bar{\lambda}_{\mathrm{t}} \bar{\lambda}_{\mathrm{b}} \in[0,1 / 4]$ (Appendix C).

- The maxima and minima values of the total dimensionless bulk- viscosity number $\Sigma_{\bar{\eta}}$ are bounded by the values of the Maxwell relation times in the bottom and the top, and in general satisfy the following inequality:

$$
\min \left\{\bar{\lambda}_{\mathrm{t}}^{( \pm)}, \bar{\lambda}_{\mathrm{b}}^{( \pm)}\right\}=\Sigma_{\bar{\eta} \min }^{( \pm)} \leq \Sigma_{\bar{\eta}}^{( \pm)} \leq \Sigma_{\bar{\eta} \max }^{( \pm)}=\max \left\{\bar{\lambda}_{\mathrm{t}}^{( \pm)}, \bar{\lambda}_{\mathrm{b}}^{( \pm)}\right\}
$$

Where the positive and negative sign comes from of a quadratic algebraic equation for the Maxwell relation times (Appendix C). 
- The dimensionless elastic ratio number is independent of the asymmetric number $\bar{\lambda}_{t} \bar{\lambda}_{b}$ and the bulk-viscous mechanism $\boldsymbol{\Sigma}_{\bar{\eta}}$ and its value is bounded by: $\mathrm{k}_{\text {min }} \leq \mathrm{k} \leq \mathrm{k}_{\max }=1 ; \mathrm{k}_{\min }<<1$ (See Appendix C).

In order to simplify the notation, in the rest of the paper the dependency of the dimensionless numbers in the materials parametric functions has been omitted, i.e., $\mathrm{b}_{2}^{*}=\mathrm{b}_{2}^{*}\left(\mathrm{k}, \bar{\lambda}_{\mathrm{t}} \bar{\lambda}_{\mathrm{b}}\right) ; \mathrm{b}_{1}^{*}=\mathrm{b}_{1}^{*}\left(\mathrm{k}, \Sigma_{\bar{\eta}}\right)$

\subsection{Mechanical Response and Energy}

\subsubsection{Curvature Moduli}

In this section we study the membrane shape response to a small amplitude oscillation of the electrical $\overline{\mathbf{E}}[\overline{\mathrm{t}} ; \overline{\mathrm{w}}]$ field $[16,18]$ :

$$
\begin{aligned}
& \overline{\mathrm{E}}[\overline{\mathrm{t}} ; \overline{\mathrm{w}}]=\cos (\overline{\mathrm{w}} \overline{\mathrm{t}}) \\
& \mathrm{b}_{2}^{*} \ddot{\overline{\mathrm{H}}}(\overline{\mathrm{t}})+\mathrm{b}_{1}^{*} \dot{\overline{\mathrm{H}}}(\overline{\mathrm{t}})+\overline{\mathrm{H}}(\overline{\mathrm{t}})=\left(1-\mathrm{kb}_{2}^{*} \overline{\mathrm{w}}^{2}\right) \cos (\overline{\mathrm{w}} \overline{\mathrm{t}})-\overline{\mathrm{w}} \sin (\overline{\mathrm{w} t})
\end{aligned}
$$

The oscillator has two inputs: $\left(1-\mathrm{kb}_{2}^{*} \overline{\mathrm{w}}^{2}\right) \cos (\overline{\mathrm{wt}})$ and $-\overline{\mathrm{w}} \sin (\overline{\mathrm{wt}})$. The response of the membrane average dimensionless curvature $\overline{\mathrm{H}}(\overline{\mathrm{t}} ; \overline{\mathrm{w}})$ to the oscillating electric field can be separated in two moduli $\overline{\mathrm{H}}_{\mathrm{io}}(\overline{\mathrm{w}})$ and $\overline{\mathrm{H}}_{\mathrm{oi}}(\overline{\mathrm{w}})$ respectively:

$$
\overline{\mathrm{H}}(\overline{\mathrm{t}} ; \overline{\mathrm{w}})=\overline{\mathrm{H}}_{\text {io }}(\overline{\mathrm{w}}) \cos (\overline{\mathrm{w} t})+\overline{\mathrm{H}}_{\mathrm{oi}}(\overline{\mathrm{w}}) \sin (\overline{\mathrm{w}} \overline{\mathrm{t}})
$$

Due to two inputs $\{\sin (\overline{\mathrm{wt}}), \cos (\overline{\mathrm{wt}})\}$ in the system, the following subscript notation for the moduli is adopted: 
(i) In-out (io) to mean in phase with cosine and out of phase with sine

(ii) Out-in (oi) to mean out of phase with cosine and in phase with sine.

Substitution of equation (28) into equation (27) we find:

$\left\{\begin{array}{l}\left(\left(1-\mathrm{b}_{2}^{*} \overline{\mathrm{w}}^{2}\right) \overline{\mathrm{H}}_{\mathrm{io}}+\overline{\mathrm{w}}_{\mathrm{oi}}\right) \cos \overline{\mathrm{w}} \mathrm{t}=\left(1-\mathrm{kb}_{2}^{*} \overline{\mathrm{w}}^{2}\right) \cos \overline{\mathrm{w} t} \\ \left(-\mathrm{b}_{1}^{*} \overline{\mathrm{w}}_{\mathrm{io}}+\left(1-\mathrm{b}_{2}^{*} \overline{\mathrm{w}}^{2}\right) \overline{\mathrm{H}}_{\mathrm{oi}}\right) \sin \overline{\mathrm{w} t}=-\overline{\mathrm{w}} \sin \overline{\mathrm{w} t}\end{array}\right\}$

Equating coefficients in the sine and cosine function, equation (29) can be expressed in a matrix form:

$\left(\begin{array}{cc}1-\mathrm{b}_{2}^{*} \overline{\mathrm{w}}^{2} & \overline{\mathrm{w}}_{1}^{*} \\ -\overline{\mathrm{w}} \mathrm{b}_{1}^{*} & 1-\mathrm{b}_{2}^{*} \overline{\mathrm{w}}^{2}\end{array}\right)\left(\begin{array}{l}\overline{\mathrm{H}}_{\mathrm{io}} \\ \overline{\mathrm{H}}_{\mathrm{oi}}\end{array}\right)=\left(\begin{array}{c}1-\mathrm{k} \mathrm{b}_{2}^{*} \overline{\mathrm{w}}^{2} \\ -\overline{\mathrm{w}}\end{array}\right)$

Solving the matrix given in (30) for $\overline{\mathrm{H}}_{\mathrm{io}}$ and $\overline{\mathrm{H}}_{\mathrm{o}}$, the following expressions for the in-out of phase and out-in phase curvature moduli are obtained:

$$
\begin{aligned}
& \overline{\mathrm{H}}_{\mathrm{io}}\left[\overline{\mathrm{w}} ; \mathrm{b}_{1}^{*}, \mathrm{~b}_{2}^{*}, \mathrm{k}\right]=\frac{1+\left(\mathrm{b}_{1}^{*}-(1+\mathrm{k}) \mathrm{b}_{2}^{*}\right) \overline{\mathrm{w}}^{2}+\mathrm{kb}_{2}^{* 2} \overline{\mathrm{w}}^{4}}{\left(1-\mathrm{b}_{2}^{*} \overline{\mathrm{w}}^{2}\right)^{2}+\left(\mathrm{b}_{1}^{*} \overline{\mathrm{w}}\right)^{2}} \\
& \overline{\mathrm{H}}_{\mathrm{oi}}\left[\overline{\mathrm{w}} ; \mathrm{b}_{1}^{*}, \mathrm{~b}_{2}^{*}, \mathrm{k}\right]=\frac{\left(\mathrm{b}_{1}^{*}-1\right) \overline{\mathrm{w}}+\left(1-\mathrm{kb}_{1}^{*}\right) \mathrm{b}_{2}^{*} \overline{\mathrm{w}}^{3}}{\left(1-\mathrm{b}_{2}^{*} \overline{\mathrm{w}}^{2}\right)^{2}+\left(\mathrm{b}_{1}^{*} \overline{\mathrm{w}}\right)^{2}}
\end{aligned}
$$

We note the fourth (third) order frequency dependency in $\overline{\mathrm{H}}_{\mathrm{io}}\left[\overline{\mathrm{w}} ; \mathrm{b}_{1}^{*}, \mathrm{~b}_{2}^{*}, \mathrm{k}\right]$ and $\overline{\mathrm{H}}_{\mathrm{oi}}\left[\overline{\mathrm{w}} ; \mathrm{b}_{1}^{*}, \mathrm{~b}_{2}^{*}, \mathrm{k}\right]$ characteristic of the second order differential eqn. (27) and the usual 
resonance term $\left(1-b_{2}^{*} \bar{w}^{2}\right)$. The numerator coefficients in eqns. $(31,32)$ are positive $\left(b_{1}^{*}-(k+1) b_{2}^{*} \geq 0 ; 1-k b_{1}^{*} \geq 0\right)$ showing that the moduli increase with frequency. The shape dynamics is characterized the complex curvature $\overline{\mathrm{H}}^{*}\left[\overline{\mathrm{w}} ; \mathrm{b}_{1}^{*}, \mathrm{~b}_{2}^{*}, \mathrm{k}\right][25,26]$ :

$\overline{\mathrm{H}}^{*}\left[\overline{\mathrm{w}} ; \mathrm{b}_{1}^{*}, \mathrm{~b}_{2}^{*}, \mathrm{k}\right]=\sqrt{\overline{\mathrm{H}}_{\text {io }}\left[\overline{\mathrm{w}} ; \mathrm{b}_{1}^{*}, \mathrm{~b}_{2}^{*}, \mathrm{k}\right]^{2}+\overline{\mathrm{H}}_{\mathrm{oi}}\left[\overline{\mathrm{w}} ; \mathrm{b}_{1}^{*}, \mathrm{~b}_{2}^{*}, \mathrm{k}\right]^{2}}$

\subsubsection{Fluid Power and Membrane Elastic Energy}

\subsubsection{Dissipation}

The average power delivered to the viscoelastic fluids $\overline{\mathrm{P}}$ is defined by the time average over a period of viscous contribution of the input electrical field $\bar{E}(\bar{t})$ multiplied by the volumetric flow, which is given by the time derivative of the curvature, i.e., $\overline{\mathrm{Q}}(\overline{\mathrm{t}})=\dot{\overline{\mathrm{H}}}(\overline{\mathrm{t}})$, where the dimensionless volumetric flow rate is given by $\bar{Q}=Q / \pi a^{3}$.

$\overline{\mathrm{P}}\left(\overline{\mathrm{w}} ; \mathrm{b}_{1}^{*}, \mathrm{~b}_{2}^{*}, \mathrm{k}\right)=\langle\overline{\mathrm{E}}(\overline{\mathrm{t}}) \cdot \overline{\mathrm{Q}}(\overline{\mathrm{t}})\rangle=\langle\overline{\mathrm{E}}(\overline{\mathrm{t}}) \cdot \dot{\overline{\mathrm{H}}}(\overline{\mathrm{t}})\rangle$

The average dimensionless power $\overline{\mathrm{P}}$, computed in Appendix $\mathrm{D}$, is given by $\overline{\mathrm{P}}\left(\overline{\mathrm{w}} ; \mathrm{b}_{1}^{*}, \mathrm{~b}_{2}^{*}, \mathrm{k}\right)=\frac{1}{2} \overline{\mathrm{w}}_{\mathrm{oi}}\left[\overline{\mathrm{w}} ; \mathrm{b}_{1}^{*}, \mathrm{~b}_{2}^{*}, \mathrm{k}\right]=\frac{1}{2} \frac{\left(\mathrm{b}_{1}^{*}-1\right) \overline{\mathrm{w}}^{2}+\left(1-\mathrm{kb}_{1}^{*}\right) \mathrm{b}_{2}^{*} \overline{\mathrm{w}}^{4}}{\left(1-\mathrm{b}_{2}^{*} \overline{\mathrm{w}}^{2}\right)^{2}+\left(\mathrm{b}_{1}^{*} \overline{\mathrm{w}}\right)^{2}}$

Eqn. (35) satisfies the following asymptotic limits for low and high dimensionless frequency respectively: 
$\operatorname{Lim}_{\overline{\mathrm{w}} \rightarrow 0} \overline{\mathrm{P}}\left(\overline{\mathrm{w}} ; \mathrm{b}_{1}^{*}, \mathrm{~b}_{2}^{*}, \mathrm{k}\right)=\frac{\left(\mathrm{b}_{1}^{*}-1\right) \overline{\mathrm{w}}^{2}}{2}, \operatorname{Lim}_{\overline{\mathrm{w}} \rightarrow \infty} \mathrm{P}\left(\overline{\mathrm{w}} ; \mathrm{b}_{1}^{*}, \mathrm{~b}_{2}^{*}, \mathrm{k}\right)=\frac{1-\mathrm{kb}_{1}^{*}}{\mathrm{~b}_{2}^{*}}$

For low values of frequency, the power contribution presents a quadratic dependence, to follow a monotonically increasing behavior for moderate frequencies, and finally a nonbounded behavior for high dimensionless frequencies.

\subsubsection{Storage}

The second quantity of the interest is the elastic membrane energy, which is defined by the average product between the square of the membrane and is given by the following expression

$\overline{\mathrm{Em}}\left(\overline{\mathrm{w}} ; \mathrm{b}_{1}^{*}, \mathrm{~b}_{2}^{*}, \mathrm{k}\right)=\langle\overline{\mathrm{H}}(\overline{\mathrm{t}} ; \overline{\mathrm{w}}) \cdot \overline{\mathrm{H}}(\overline{\mathrm{t}} ; \overline{\mathrm{w}})\rangle=\left\langle(\overline{\mathrm{H}}(\overline{\mathrm{t}} ; \overline{\mathrm{w}}))^{2}\right\rangle$

In expression (37) the dimensionless elastic membrane energy is $\overline{\mathrm{Em}}=\mathrm{Em} / 2 \pi \mathrm{a}^{2} \mathrm{~L} \Sigma_{\mathrm{G}}$. The average value $\overline{\operatorname{Em}}\left(\overline{\mathrm{w}} ; \mathrm{b}_{1}^{*}, \mathrm{~b}_{2}^{*}, \mathrm{k}\right)$, computed in Appendix $\mathrm{D}$, is given by

$$
\operatorname{Em}\left(\overline{\mathrm{w}} ; \mathrm{b}_{1}^{*}, \mathrm{~b}_{2}^{*}, \mathrm{k}\right)=\frac{1}{2}\left(\left(\overline{\mathrm{H}}_{\mathrm{io}}\left(\overline{\mathrm{w}} ; \mathrm{b}_{1}^{*}, \mathrm{~b}_{2}^{*}, \mathrm{k}\right)\right)^{2}+\left(\overline{\mathrm{H}}_{\mathrm{oi}}\left(\overline{\mathrm{w}} ; \mathrm{b}_{1}^{*}, \mathrm{~b}_{2}^{*}, \mathrm{k}\right)\right)^{2}\right)=\frac{1}{2}\left(\overline{\mathrm{H}}^{*}\left(\overline{\mathrm{w}} ; \mathrm{b}_{1}^{*}, \mathrm{~b}_{2}^{*}, \mathrm{k}\right)\right)^{2}
$$

The elastic membrane energy is proportional to the square of the dimensionless frequency and the sum of quadrative dependence of the curvatures moduli and the inertial mechanism through characteristic dimensionless numbers. The asymptotic limits of equation (55) are:

$\lim _{\overline{\mathrm{w}} \rightarrow 0} \operatorname{Em}\left(\overline{\mathrm{w}} ; \mathrm{b}_{1}^{*}, \mathrm{~b}_{2}^{*}, \mathrm{k}\right)=1 ; \quad \lim _{\overline{\mathrm{w}} \rightarrow \infty} \overline{\mathrm{H}}^{*}\left(\overline{\mathrm{w}} ; \mathrm{b}_{1}^{*}, \mathrm{~b}_{2}^{*}, \mathrm{k}\right)=\mathrm{k}^{2}$ 


\subsubsection{Q-factor}

The $\mathrm{Q}$-factor is the ratio between elastic membrane energy $\overline{\mathrm{Em}}$ and the power $\overline{\mathrm{P}}$ :

$\mathrm{Q}\left(\overline{\mathrm{w}} ; \mathrm{b}_{1}^{*}, \mathrm{~b}_{2}^{*}, \mathrm{k}\right)=\frac{\overline{\mathrm{Em}}\left(\overline{\mathrm{w}} ; \mathrm{b}_{1}^{*}, \mathrm{~b}_{2}^{*}, \mathrm{k}\right)}{\overline{\mathrm{P}}\left(\overline{\mathrm{w}} ; \mathrm{b}_{1}^{*}, \mathrm{~b}_{2}^{*}, \mathrm{k}\right)}=\frac{\overline{\mathrm{H}}^{* 2}}{\overline{\mathrm{wH}}_{\mathrm{oi}}}$

The $\mathrm{Q}\left(\overline{\mathrm{w}} ; \mathrm{b}_{1}^{*}, \mathrm{~b}_{2}^{*}, \mathrm{k}\right)$ factor is a measure of the relative importance of dissipative and storage processes. It is important to note that the $\mathrm{Q}\left(\overline{\mathrm{w}} ; \mathrm{b}_{1}^{*}, \mathrm{~b}_{2}^{*}, \mathrm{k}\right)$ factor depend on the bulk, inertial and elastic ratio mechanisms $\left\{\mathrm{b}_{1}^{*}, \mathrm{~b}_{2}^{*}\right\}$, though dimensionless numbers $\left\{\bar{\lambda}_{\mathrm{t}} \bar{\lambda}_{\mathrm{b}}, \boldsymbol{\Sigma}_{\bar{\eta}}, \mathrm{k}\right\}$. When the $\mathrm{Q}$ factor is greater than one, i.e., $\mathrm{Q}\left(\overline{\mathrm{w}} ; \mathrm{b}_{1}^{*}, \mathrm{~b}_{2}^{*}, \mathrm{k}\right)>>1$ the elastic mechanism dominates over dissipative processes $\overline{\operatorname{Em}}\left(\overline{\mathrm{W}}_{\text {res }} ; \mathrm{b}_{1}^{*}, \mathrm{~b}_{2}^{*}, \mathrm{k}\right)>>\overline{\mathrm{P}}\left(\overline{\mathrm{W}}_{\text {res }} ; \mathrm{b}_{1}^{*}, \mathrm{~b}_{2}^{*}, \mathrm{k}\right)$. The second case is when $\mathrm{Q}\left(\overline{\mathrm{w}} ; \mathrm{b}_{1}^{*}, \mathrm{~b}_{2}^{*}, \mathrm{k}\right)<<1$, in this case, the dissipative process are dominated by the elastic mechanism, i.e., $\overline{\mathrm{P}}\left(\overline{\mathrm{w}} ; \mathrm{b}_{1}^{*}, \mathrm{~b}_{2}^{*}, \mathrm{k}\right)<<\overline{\mathrm{Em}}\left(\overline{\mathrm{w}} ; \mathrm{b}_{1}^{*}, \mathrm{~b}_{2}^{*}, \mathrm{k}\right)$. The last case is when the $\mathrm{Q}\left(\overline{\mathrm{w}} ; \mathrm{b}_{1}^{*}, \mathrm{~b}_{2}^{*}, \mathrm{k}\right)=1$ factor is equal to the unity and the dissipative and elastic mechanism are equal, i.e., $\overline{\operatorname{Em}}\left(\overline{\mathrm{W}}_{\text {res }} ; \mathrm{b}_{1}^{*}, \mathrm{~b}_{2}^{*}, \mathrm{k}\right)=\overline{\mathrm{P}}\left(\overline{\mathrm{w}}_{\text {res }} ; \mathrm{b}_{1}^{*}, \mathrm{~b}_{2}^{*}, \mathrm{k}\right)$. For simplicity in what follow we use for the power and elastic membrane energy : $\overline{\mathrm{P}}(\overline{\mathrm{w}}) \equiv \overline{\mathrm{P}}\left(\overline{\mathrm{w}} ; \mathrm{b}_{1}^{*}, \mathrm{~b}_{2}^{*}, \mathrm{k}\right) ; \overline{\operatorname{Em}}(\overline{\mathrm{w}}) \equiv \overline{\operatorname{Em}}\left(\overline{\mathrm{w}} ; \mathrm{b}_{1}^{*}, \mathrm{~b}_{2}^{*}, \mathrm{k}\right)$; $\mathrm{Q}(\overline{\mathrm{w}}) \equiv \mathrm{Q}\left(\overline{\mathrm{w}} ; \mathrm{b}_{1}^{*}, \mathrm{~b}_{2}^{*}, \mathrm{k}\right)$

\subsubsection{Asymptotic Regimes and Resonance}

The curvature dynamics' terminal and high frequency regimes are found from eqn. (27) to be:

$$
\overline{\mathrm{w}} \rightarrow 0: \overline{\mathrm{H}}(\overline{\mathrm{t}})=\overline{\mathrm{E}}(\overline{\mathrm{t}}), \quad \overline{\mathrm{w}} \rightarrow \infty: \ddot{\overline{\mathrm{H}}}(\overline{\mathrm{t}})=\mathrm{k} \ddot{\overline{\mathrm{E}}}(\overline{\mathrm{t}})
$$


such that at low frequency only the membrane elasticity determines the purely in-phase response while at high frequency the purely in-phase curvature is a function of the elasticity

ratio $k$ (see eqn.(27)). Next we give parametric details on $\left(\overline{\mathrm{H}}_{\mathrm{io}}, \overline{\mathrm{H}}_{\mathrm{oi}}\right)$, power and membrane elastic energy contributions and finally a resonance analysis of the mechanical system.

\subsubsection{Resonance frequency}

The resonance frequency $\overline{\mathrm{w}}_{\text {res }}$, read from denominator of equation eqns. (30-31), is given by the following expression

$$
\overline{\mathrm{w}}_{\mathrm{res}}=\frac{1}{\sqrt{\mathrm{b}_{2}^{*}}}=\sqrt{\frac{\mathrm{k}}{\bar{\lambda}_{\mathrm{t}} \bar{\lambda}_{\mathrm{b}}}}
$$

The resonance frequency $\overline{\mathrm{w}}_{\text {res }}$ decreases with the inertia mechanism inertia through dimensionless parametric function $b_{2}^{*}$ which depends on the memory of the system $\bar{\lambda}_{\mathrm{t}} \bar{\lambda}_{\mathrm{b}}$ and the elastic ratio $\mathrm{k}$.

\subsubsection{In-out phase curvature $\overline{\mathrm{H}}_{\mathrm{i}}$}

Using eqn. (31) the terminal and high frequency regimes give

$$
\lim _{\overline{\mathrm{w}} \rightarrow 0} \overline{\mathrm{H}}_{\mathrm{io}}[\overline{\mathrm{w}}]=1 ; \overline{\mathrm{H}}_{\text {iores }}=\frac{1}{\mathrm{~b}_{1}^{*}} ; \lim _{\overline{\mathrm{w}} \rightarrow \infty} \overline{\mathrm{H}}_{\mathrm{io}}[\overline{\mathrm{w}}]=\mathrm{k}<<1
$$

In equation (43-b) the resonance curvature moduli is given by $\overline{\mathrm{H}}_{\mathrm{iores}}=\overline{\mathrm{H}}_{\mathrm{io}}\left[\overline{\mathrm{W}}_{\mathrm{res}}\right]$.

It is important to note, that the purely elastic terminal regime the membrane curvature is inphase with the field. 


\subsubsection{Out-in phase curvature $\overline{\mathrm{H}}_{\mathrm{oi}}$}

Using eqn. (32) the terminal and high frequency regimes give $\lim _{\overline{\mathrm{w}} \rightarrow 0} \overline{\mathrm{H}}_{\mathrm{oi}}[\overline{\mathrm{w}}]=\left(\mathrm{b}_{1}^{*}-1\right) \overline{\mathrm{w}} ; \overline{\mathrm{H}}_{\mathrm{oires}}=(1-\mathrm{k}) \frac{\sqrt{\mathrm{b}_{2}^{*}}}{\mathrm{~b}_{1}^{*}} ; \lim _{\overline{\mathrm{w}} \rightarrow \infty} \overline{\mathrm{H}}_{\mathrm{oi}}[\overline{\mathrm{w}}]=0$

In the terminal regime $\overline{\mathrm{H}}_{\mathrm{oi}}$ scales like $\overline{\mathrm{w}}$ with a slope $\left(\mathrm{b}_{1}^{*}-1\right)>0$ given by the viscosity coefficient. At large frequency the curvature is in-phase and hence the out-in phase curvature decreases monotonically until to zero, i.e., $\overline{\mathrm{H}}_{\mathrm{oi}}[\overline{\mathrm{w}}]=0$.

\subsubsection{Complex curvature $\overline{\mathrm{H}}^{*}$}

Using eqns. (27-29) the corresponding asymptotic values for $\overline{\mathrm{H}}^{*}$ are $\lim _{\overline{\mathrm{w}} \rightarrow 0} \overline{\mathrm{H}}^{*}=\sqrt{1+\left(\mathrm{b}_{1}^{*}-1\right)^{2} \overline{\mathrm{w}}^{2}} ; \overline{\mathrm{H}}_{\text {res }}^{*}=\mathrm{b}_{1}^{*-1} \sqrt{1+(1-\mathrm{k})^{2} \mathrm{~b}_{2}^{*}} ; \lim _{\overline{\mathrm{w}} \rightarrow \infty} \overline{\mathrm{H}}^{*}=\mathrm{k} \quad$ (46a-c)

which shows that increasing the viscous coefficient $b_{1}^{*}$ increase the $\overline{\mathrm{H}}^{*}(\overline{\mathrm{w}} \rightarrow 0)$,

and decreases $\overline{\mathrm{H}}_{\text {res }}^{*}$ increasing the inertia coefficient $\mathrm{b}_{2}^{*}$ increase the resonant peak amplitude $\overline{\mathrm{H}}_{\text {res }}^{*}$, and increasing the elasticity ratio k increase $\overline{\mathrm{H}}^{*}(\overline{\mathrm{w}} \rightarrow \infty)$ but decreases $\overline{\mathrm{H}}_{\text {res }}^{*}$ as expected.

\subsubsection{Energy considerations}

\section{a) Power}

The resonance power can be computed from equation (39) and equations (44a, b) for the average curvature: 
$\overline{\mathrm{P}}_{\text {res }}=\frac{1}{2} \overline{\mathrm{W}}_{\text {res }} \overline{\mathrm{H}}_{\text {oires }}=\frac{1}{2 \mathrm{~b}_{1}^{*}}(1-\mathrm{k})$

Using the definition for $b_{1}^{*}$, the following expression for the resonance power in terms of the primitive dimensionless parameters is obtained:

$\overline{\mathrm{P}}_{\text {res }}\left[\mathrm{k}, \Sigma_{\bar{\eta}}, \bar{\lambda}_{\mathrm{t}} \bar{\lambda}_{\mathrm{b}}\right]=\frac{1}{2} \frac{\mathrm{k}}{\mathrm{k}+(1-\mathrm{k}) \Sigma_{\bar{\eta}}}(1-\mathrm{k})$

In the same form, the elastic membrane energy can be computed from equation (41) and (44a,

b)

b) Elastic membrane energy

$\overline{\mathrm{E}}_{\text {mres }}=\left\langle\left(\overline{\mathrm{H}}\left(\overline{\mathrm{w}}_{\text {res }}\right)\right)^{2}\right\rangle=\frac{1}{2 \mathrm{~b}_{1}^{* 2}}\left(1+(1-\mathrm{k})^{2} \mathrm{~b}_{2}^{* 2}\right)$

In terms of the dimensionless numbers, the elastic membrane energy is given by:

$\overline{\mathrm{E}}_{\text {mres }}\left[\mathrm{k}, \Sigma_{\overline{\bar{\eta}}}, \bar{\lambda}_{\mathrm{t}} \bar{\lambda}_{\mathrm{b}}\right]=\frac{1}{2} \frac{\mathrm{k}}{\left(\mathrm{k}+(1-\mathrm{k}) \Sigma_{\bar{\eta}}\right)^{2}}\left(\mathrm{k}+(1-\mathrm{k})^{2} \bar{\lambda}_{\mathrm{t}} \bar{\lambda}_{\mathrm{b}}\right)$

It is important to note that the both expressions, power and membrane elastic energy depend on the dimensionless numbers thorough characteristic primitive variables.

\subsubsection{Resonance $Q$ factor}

In other hand, one important quantity is the resonant energy ratio $r$ and is given by: 
$\mathrm{Q}_{\text {res }}\left(\mathrm{b}_{1}^{*}, \mathrm{~b}_{2}^{*}, \mathrm{k}\right)=\frac{\overline{\mathrm{Em}}_{\text {res }}\left[\mathrm{k}, \mathrm{b}_{2}^{*}\right]}{\overline{\mathrm{P}}_{\text {res }}\left[\mathrm{k}, \mathrm{b}_{1}^{*}\right]}=\frac{1+(1-\mathrm{k})^{2} \mathrm{~b}_{2}^{* 2}}{\mathrm{~b}_{1}^{*}(1-\mathrm{k})}$

In terms of terms of the dimensionless numbers, the $Q$ factor takes the following form:

$\mathrm{Q}_{\text {res }}\left(\mathrm{k} ; \Sigma_{\bar{\eta}}, \bar{\lambda}_{\mathrm{t}} \bar{\lambda}_{\mathrm{b}}\right)=\frac{\overline{\mathrm{Em}}_{\text {res }}\left[\mathrm{k}, \bar{\lambda}_{\mathrm{t}} \bar{\lambda}_{\mathrm{b}}\right]}{\mathrm{P}_{\text {res }}\left[\mathrm{k}, \Sigma_{\bar{\eta}}\right]}=\frac{\mathrm{k}+(1-\mathrm{k})^{2} \bar{\lambda}_{\mathrm{t}} \bar{\lambda}_{\mathrm{b}}}{(1-\mathrm{k})\left(\mathrm{k}+(1-\mathrm{k}) \Sigma_{\bar{\eta}}\right)}$

Three important regimens are visualized:

(i) Inviscid fluid:

$\operatorname{Lim}_{\left(\bar{\lambda}_{\mathrm{t}} \bar{\lambda}_{\mathrm{b}}, \Sigma_{\bar{\eta}}\right) \rightarrow(0,0)} \mathrm{Q}_{\mathrm{res}}\left(\mathrm{k} ; \Sigma_{\bar{\eta}}, \bar{\lambda}_{\mathrm{t}} \bar{\lambda}_{\mathrm{b}}\right) \rightarrow \frac{1}{1-\mathrm{k}}$

(ii) Viscous Fluid:

$\operatorname{Lim}_{\left(\bar{\lambda}_{\mathrm{t}} \bar{\lambda}_{\mathrm{b}}, \Sigma_{\bar{\eta}}\right) \rightarrow(0,1)} \mathrm{Q}_{\mathrm{res}}\left(\mathrm{k} ; \Sigma_{\overline{\bar{\eta}}}, \bar{\lambda}_{\mathrm{t}} \bar{\lambda}_{\mathrm{b}}\right) \rightarrow \frac{\mathrm{k}}{1-\mathrm{k}}$

(iii) Symmetric case: Viscoelastic/Membrane /Viscoelastic

$\operatorname{Lim}_{\left(\bar{\lambda}_{\mathrm{t}} \bar{\lambda}_{\mathrm{b}}, \Sigma_{\bar{\eta}}\right) \rightarrow(0.25,0.5)} \mathrm{Q}_{\text {res }}\left(\mathrm{k} ; \Sigma_{\bar{\eta}}, \bar{\lambda}_{\mathrm{t}} \bar{\lambda}_{\mathrm{b}}\right)=\frac{1}{2} \frac{1+\mathrm{k}}{1-\mathrm{k}}$

(iv) Stiffness membrane

$\operatorname{Lim}_{\mathrm{k} \rightarrow 1} \mathrm{Q}_{\text {res }}\left(\mathrm{k} ; \Sigma_{\bar{\eta}}, \bar{\lambda}_{\mathrm{t}} \bar{\lambda}_{\mathrm{b}}\right) \rightarrow \infty$

It is important to note that equations (53-56) show particular cases of the parametric space governed by equation (52). When the total viscosity of the system is less than one, the energy 
ratio is completely determined by equation (51) which describes a linear plot with slope $\bar{\lambda}_{\mathrm{t}} \bar{\lambda}_{\mathrm{b}}$. In the case of a viscous fluid, energy ratio is given by inverse of the product between the viscoelastic dimensionless Maxwell relaxation time. The third case (equation 54) corresponds to the symmetric case $\bar{\lambda}_{\mathrm{t}}=\bar{\lambda}_{\mathrm{b}}=1 / 2$, when the system present similar viscoelastic phases. Finally, It is important to note, that equation (56) is not bounded for the maxima value of the elastic ratio $\mathrm{k}=1$.

In the following section, the main results of the present work using the equations obtained for the curvature moduli, power and elastic membrane energy and resonance are presented.

\subsection{Results}

In this section the mechanical response (curvature moduli) and energetic considerations (dissipative viscous mechanism and elastic storage processes) as a function of the dimensionless frequency are presented. The former corresponds to a generic point in the material phase space $\mathbf{S} \in \mathbf{R}^{3}$ (Fig. (4)) where the membrane elasticity ( $\left.b_{0}\right)$, the viscosity coefficient $\left(b_{1}\right)$ and the inertial coefficient $\left(b_{2}\right)$ have significant contributions. The latter corresponds to a point $\mathbf{S}$ in Fig.(5) close to the viscous/membrane elasticity material plane in which inertial coefficient is relatively negligible. The results are computed using eqns $(31,32)$ for the in-out phase and out-in phase moduli and eqns. $(35,38)$ for the resonance power and elastic energy membrane. The resonance properties: (i) frequency, (ii) power, (iii) elasticmembrane energy, and (iv) resonance ratio were computed using eqns. (43, 46-49). The value of the dimensionless numbers and parametric functions (22-24) were chosen in order to satisfy the mathematical and physical requirements given by eqn. (25) and appendix C. Finally, in this 
section the principal analysis is centered in the study of three different mechanism: (i) elastic ratio, (ii) bulk-viscous mechanism and (iii) memory asymmetry in the bulk phases.

\subsubsection{Frequency Response}

Figure 22 shows the dimensionless curvature moduli $\left\{\overline{\mathrm{H}}_{\mathrm{io}}, \overline{\mathrm{H}}_{\mathrm{oi}}\right\}$, power $\overline{\mathrm{P}}$ and elastic membrane energy $\overline{\mathrm{E}}_{\mathrm{m}}$ as a function of dimensionless frequency $\overline{\mathrm{w}}$. The curvature frequency response is that of a second order oscillator, with $\overline{\mathrm{H}}_{\mathrm{i} \text { o }}$ displaying a low and high frequency plateaus (in-phase regime, eqns. $(35 a, b))$ and an intermediate power law region $\overline{\mathrm{H}}_{\mathrm{i} 0} \propto \overline{\mathrm{w}}^{\mathrm{n}}$, where $\mathrm{n} \approx 1.41$. The out-in phase curvature $\overline{\mathrm{H}}_{\mathrm{oi}}$ displays a single pulse $\overline{\mathrm{H}}_{\text {oires }}\left[\overline{\mathrm{W}}_{\text {res }}=0.73\right] \cong 0.44$ (eqn. (38)) centered at the resonant frequency $\overline{\mathrm{W}}_{\text {res }}=0.73$ (eqns.(36a)), where $\overline{\mathrm{H}}_{\mathrm{i} \mathrm{o}}=\overline{\mathrm{H}}_{\mathrm{oi}} \cong 0.5$ at $\overline{\mathrm{W}}_{\mathrm{res}}=0.73$. The membrane elastic energy ( $\overline{\mathrm{E}}_{\mathrm{m}}$ in Figure 22) shows $(\bar{w}=0.5)$ a low frequency plateau followed by a power law decreasing behavior $\overline{\mathrm{E}}_{\mathrm{m}} \propto \overline{\mathrm{W}}^{-\mathrm{n}}$, where $\mathrm{n} \approx 2$, and it reaches a zero value for frequencies greater than one.

Physically, this parameter is associated with the energy stored by the membrane, so in the low frequency region the membrane behaves as a perfect solid with no energy dissipation. The power ( $\overline{\mathrm{P}}$ in Figure 20) shows a monotonically increasing behavior with an inflection point at a frequency of approximately $\overline{\mathrm{w}} \cong 1$, followed by an asymptotic plateau. This parameter is associated with the energy per unit of time delivered to the fluid by the oscillating the membrane. A crossover point is observed at a frequency $\overline{\mathrm{W}}_{\text {cross }} \cong 2.1$, where $\overline{\mathrm{P}}=\overline{\mathrm{E}}_{\mathrm{m}} \cong 0.23$. At frequencies below $\overline{\mathrm{w}}<2$ the membrane exhibits a predominantly elastic behavior (the energy 
storage mechanism dominates over the dissipation one), whereas the viscous behavior dominates at frequencies above $\overline{\mathrm{w}}>2$. This could be used to predict the optimum membrane performance which for this case is located in the region of moderate frequencies, i.e., $\overline{\mathrm{W}}_{0} \in(0.1,10)$. The inset in Figure 20 shows a zoom of the power mechanism for the minimum viscosity value $\Sigma_{\bar{\eta} \text { min }}=0.101$. Figure 20 shows that the power $\overline{\mathrm{P}}$ does not present a resonance behavior as in others second order mechanical systems. This effect is due to the selected parameters. As will be seen below, the power resonance behavior is reached in the particular case when one the bulk phases is an inelastic liquid or inviscid liquid.

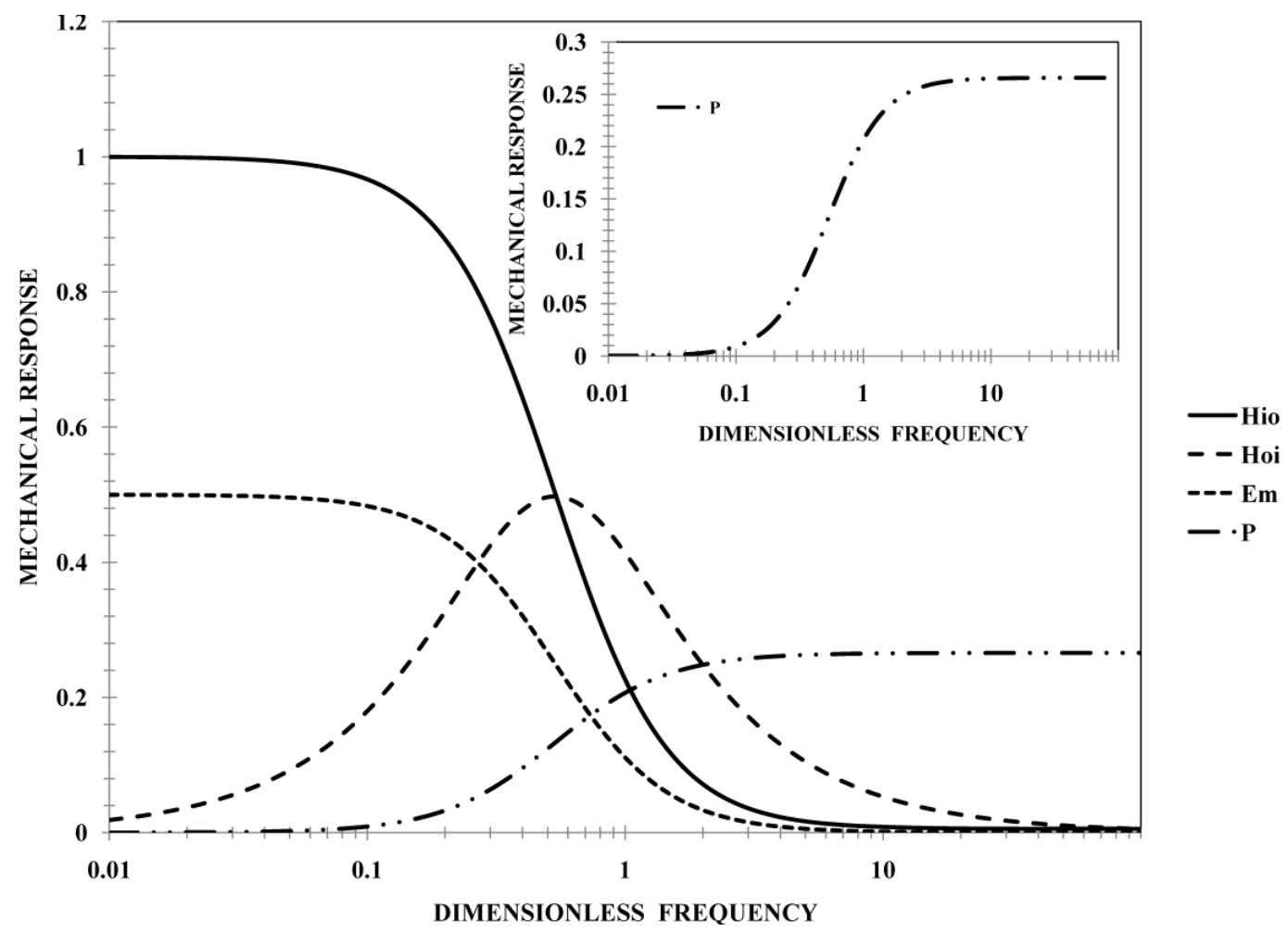

Figure 20 - Mechanical response of the curvature moduli, power and elastic energy as a function of the dimensionless frequency. The parametric values are: (i) $\bar{\lambda}_{\mathrm{t}} \bar{\lambda}_{\mathrm{b}}=0.01$, (ii) $\Sigma_{\bar{\eta}}=0.01$, (iii) $\mathrm{k}=0.0054$, and the corresponding dimensionless functions are: (iv) $b_{1}^{*}=2.84$ and $b_{2}^{*}=1.85$. 


\subsubsection{Membrane effect (k)}

Here we explore the role of increasing $k$, retaining $\bar{\lambda}_{t} \bar{\lambda}_{b}=0.01, \Sigma_{\bar{\eta}}=0.01$. Figure 23 shows the dimensionless curvature moduli $\left\{\overline{\mathrm{H}}_{\mathrm{io}}, \overline{\mathrm{H}}_{\mathrm{oi}}\right\}$, power and elastic-membrane energy $\left\{\overline{\mathrm{P}}, \overline{\mathrm{E}}_{\mathrm{m}}\right\}$ as a function of the dimensionless frequency $\overline{\mathrm{w}}$. The overall frequency response and trends of $\overline{\mathrm{H}}_{\mathrm{io}}, \overline{\mathrm{H}}_{\mathrm{oi}}, \overline{\mathrm{P}}$ and $\overline{\mathrm{E}}_{\mathrm{m}}$ are similar to those of in Figure 21, the only important effect is a shift of all the curves to the right; for example the resonance frequency is reached at $\overline{\mathrm{W}}_{\text {res }}=1 / \sqrt{\mathrm{b}_{2}^{*}}=3.16$, and the corresponding resonance value of the $\overline{\mathrm{H}}_{\mathrm{oi}}$ module is given by: $\overline{\mathrm{H}}_{\text {oires }}\left[\overline{\mathrm{W}}_{\text {res }}=3.16\right]=0.9174$. It is important to note that the maxima value does not coincide with the resonance value of the curvature moduli $\overline{\mathrm{H}}_{\mathrm{oi}}$, so the maxima value of the out-in phase curvature module is given by: $\overline{\mathrm{H}}_{\text {oimax }}\left[\overline{\mathrm{W}}_{\max }=9.91\right]=0.4547$. On the other hand, the corresponding crossover point $(\overline{\mathrm{Em}}=\overline{\mathrm{P}})$ is reached to a non-dimensional frequency $\overline{\mathrm{W}}_{\text {cross }}=1 / \sqrt{\mathrm{b}_{1}^{*}}=0.96$, and the initial plateau region of $\overline{\mathrm{H}}_{\mathrm{io}}$ and the membrane elastic energy are extended now up to a frequency close to one, i.e., $\overline{\mathrm{w}}_{\text {cross }}=1 / \sqrt{\mathrm{b}_{1}^{*}}=0.96$. It is important to note that, the changes in the resonance frequency and energy cross over point are given by:

$$
\begin{aligned}
& \Delta \overline{\mathrm{W}}_{\text {res }}=\overline{\mathrm{W}}_{\text {res }}(\mathrm{k}=0.1)-\overline{\mathrm{W}}_{\text {res }}(\mathrm{k}=0.0054) \cong 2.43 \text { and } \\
& \Delta \overline{\mathrm{W}}_{\text {cross }}=\overline{\mathrm{W}}_{\text {cross }}(\mathrm{k}=0.1)-\overline{\mathrm{W}}_{\text {cross }}(\mathrm{k}=0.0054) \cong 0.36 \text {, respectively. }
\end{aligned}
$$

Another important effect is that $\overline{\mathrm{H}}_{\text {io }}$ reaches an asymptotic behavior given by eqn. (49b), which only is determined by the elastic ratio $\mathrm{k}=0.1$ at high frequencies. The decreasing 
power law region for $\overline{\mathrm{H}}_{\mathrm{i} o} \propto \overline{\mathrm{W}}^{\mathrm{n}}$ and for $\overline{\mathrm{Em}}_{\mathrm{w}}^{\mathrm{w}}$ lies the 1-10 frequency range, where $\mathrm{n} \approx 1.40$ and $\mathrm{n} \approx 2$ respectively. Comparing Figures $\mathbf{2 0}$ and $\mathbf{2 1}$, it is seen that the net effect of increasing $\mathrm{k}$ (increasing the membrane elasticity) is to shift the crossover frequency and the maximum in the out-of-phase membrane curvature to the right which is rather logical when having a stiffer elastic membrane. The effect of increasing $k$ on the power delivered is dramatic showing here a four-fold increase at a dimensionless frequency of 100 . However, the membrane elasticity is not the only important factor in increasing the power delivered, as it will be showed in the following figure.

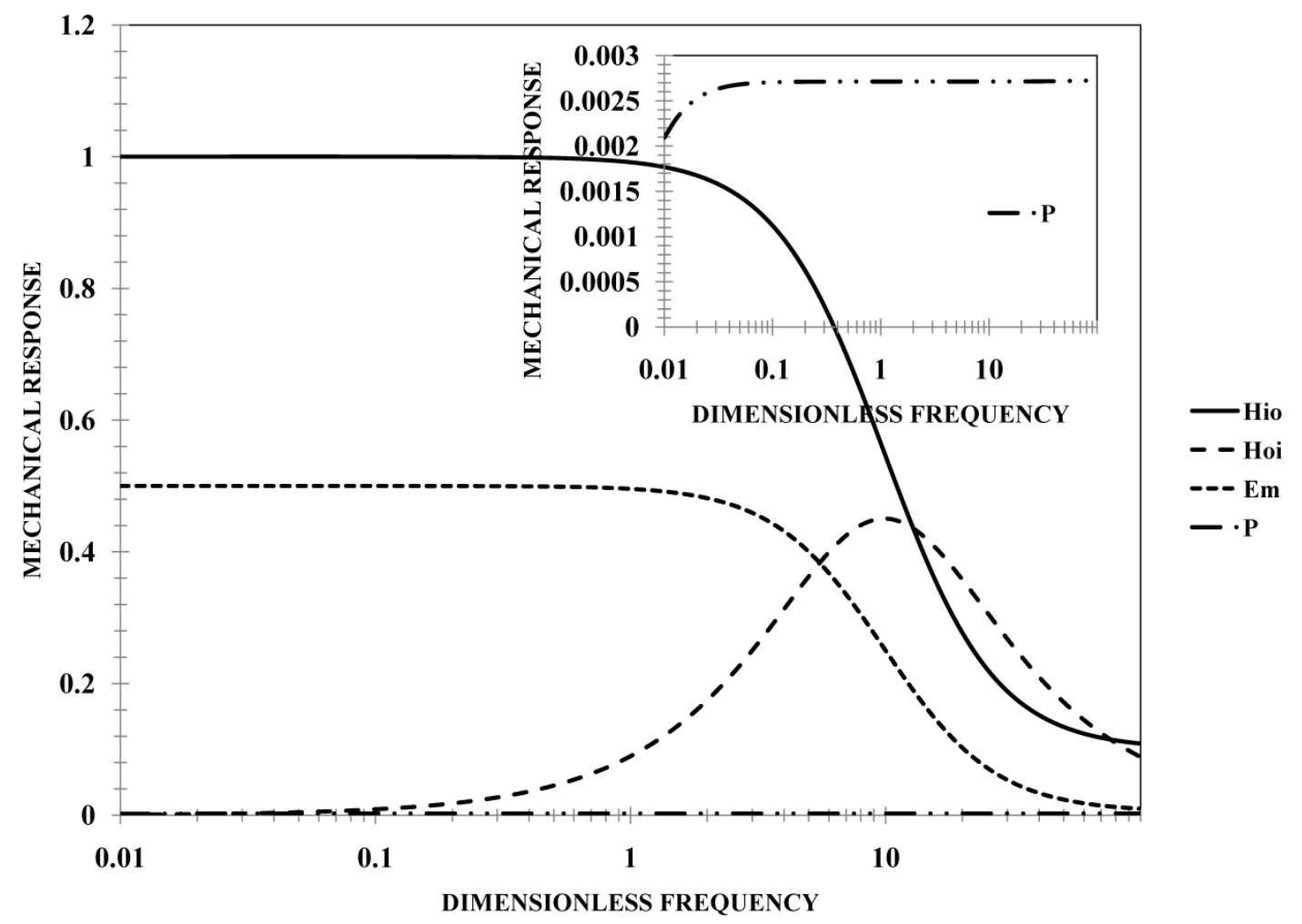

Figure 21 - Shows the mechanical response of the curvature moduli, power and elastic energy as a function of the dimensionless frequency. The dimensionless numbers used in the simulations are: (i) $\bar{\lambda}_{\mathrm{t}} \bar{\lambda}_{\mathrm{b}}=0.01$, (ii) $\Sigma_{\bar{\eta}}=0.01$, (iii) $\mathrm{k}=0.1$, and the corresponding values of the dimensionless functions are: (iv) $\mathrm{b}_{1}^{*}=5.5$ and $\mathrm{b}_{2}^{*}=0.1$ 


\subsubsection{Bulk viscous effect $\left(\Sigma_{\bar{\eta}}\right)$}

Here we explore the effect of increasing viscosity to $\Sigma_{\bar{\eta}}=0.5$, retaining $\bar{\lambda}_{\mathrm{t}} \bar{\lambda}_{\mathrm{b}}=0.01, \mathrm{k}=0.1$.

Figure 22 shows the dimensionless curvature moduli $\left\{\overline{\mathrm{H}}_{\mathrm{io}}, \overline{\mathrm{H}}_{\mathrm{oi}}\right\}$, power and elastic-membrane energy $\left\{\overline{\mathrm{P}}, \overline{\mathrm{E}}_{\mathrm{m}}\right\}$ as a function of the dimensionless frequency $\overline{\mathrm{w}}$. When the bulk-viscous effects increase in the system, the low frequency plateaus for the out-in phase and in-out of phase curvature moduli and elastic membrane energy disappears.

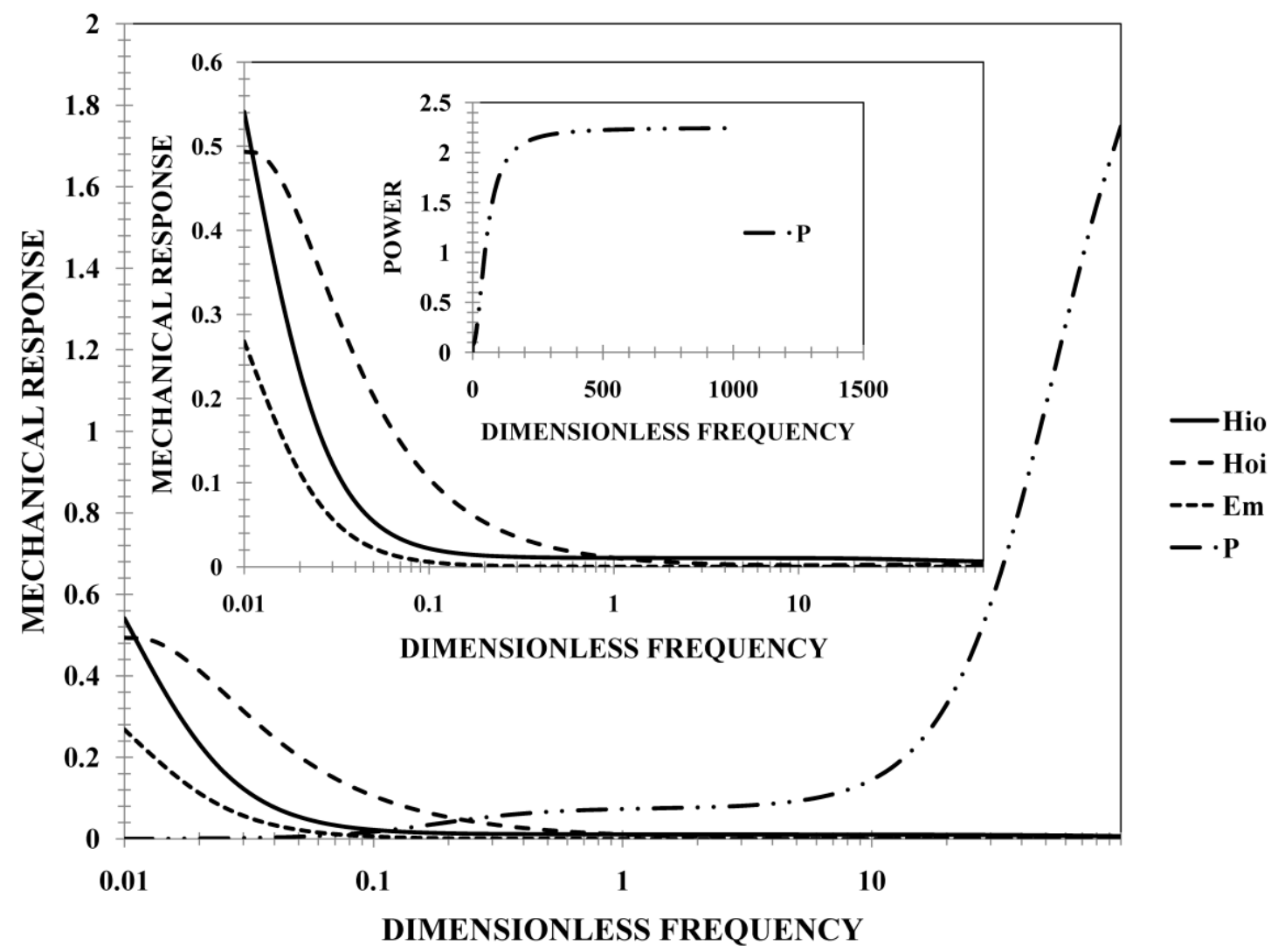

Figure 22 - Mechanical response of the curvature moduli, power and elastic energy as a function of the dimensionless frequency. The dimensionless numbers used in the simulations are: (i) $\bar{\lambda}_{\mathrm{t}} \bar{\lambda}_{\mathrm{b}}=0.01$, (ii) $\Sigma_{\bar{\eta}}=0.5$, (iii) $\mathrm{k}=0.1$, and the corresponding values of the dimensionless functions are: (iv) $b_{1}^{*}=5.5$ and $\mathrm{b}_{2}^{*}=5.0$. 
Regarding the power contribution contributions (dissipative process), the maxima value show in Figure 22 disappears. The effects of the increase in the bulk viscous mechanism in the system can be explained as the curves being shifted to lower frequencies. So, in this frequency range we observe only the power law behavior of Em and $\overline{\mathrm{H}}_{\text {io }}$. The maximum in Hoi is now at very low frequencies, i.e., $\overline{\mathrm{w}}_{0} \in(0,0.1) \subset \mathrm{R}$. So, increasing the bulk viscous mechanism value has a negative effect in the power delivered, as the power has decreased from 100 (Figure 21) to about 21 (Figure 22) for the same dimensionless frequency (100). Physically, this means that the viscosity has a negative effect in the power conversion efficiency which is again logical having a more viscous media representing a barrier for mechanical propagation.

\subsubsection{Memory effect $\left(\bar{\lambda}_{t} \bar{\lambda}_{\mathrm{b}}\right)$}

Figure 23 shows the dimensionless in-out of phase $\overline{\mathrm{H}}_{\text {io }}$, out-in phase $\overline{\mathrm{H}}_{\mathrm{oi}}$ curvature moduli, power energy am membrane elastic energy as a function of the dimensionless frequency $\overline{\mathrm{w}}$ for $\mathrm{b}_{2}^{*}=49.75, \mathrm{~b}_{1}^{*}=10^{2}, \mathrm{k}=5 \times 10^{-3}$. The overall behavior of the Figure 20 is practically the same to the Figure 17, the effects of the maximum value of the memory do not affect the general behavior of the system. 


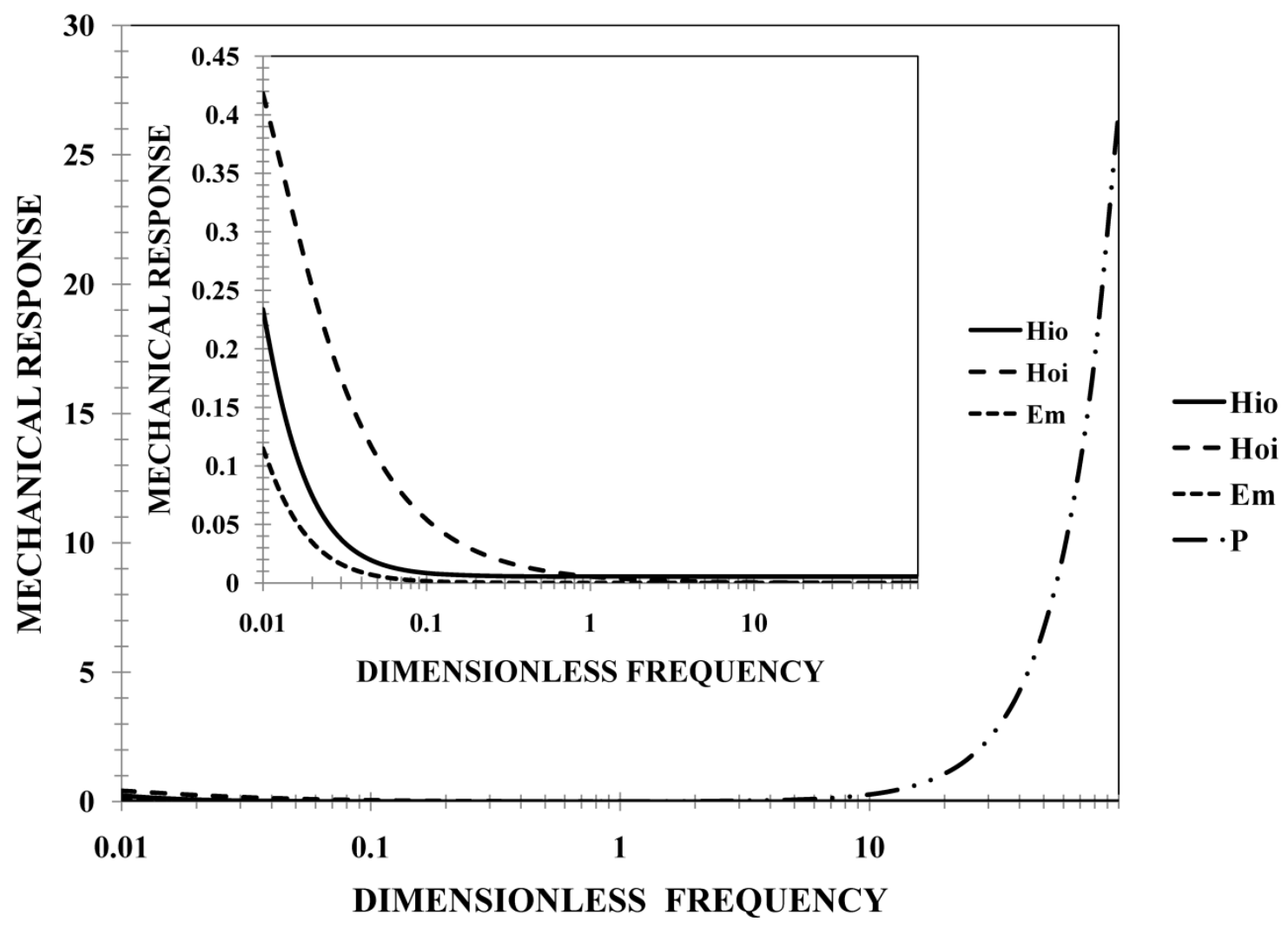

Figure 23 - Shows the mechanical response of the curvature moduli, power and elastic energy as a function of the dimensionless frequency. The dimensionless numbers used in the simulations are: (i) $\bar{\lambda}_{\mathrm{t}} \bar{\lambda}_{\mathrm{b}}=0.25$, (ii) $\Sigma_{\bar{\eta}}=0.5$, (iii) $\mathrm{k}=0.1$, and the corresponding values of the dimensionless functions are: (iv) $b_{1}^{*}=5.5$ and $b_{2}^{*}=2.0$, with $b_{1}^{*}>b_{2}^{*}$.

\subsubsection{Q factor}

Figure 24 shows the $\mathrm{Q}$ factor versus dimensionless frequency for different values of memory and bulk-viscous mechanism for $\mathrm{k}=0.0054$, and (i) $\bar{\lambda}_{\mathrm{t}} \bar{\lambda}_{\mathrm{b}}=0.01$ and $\Sigma_{\bar{\eta}}: 0.0101,0.5,0.9898$, (ii) $\bar{\lambda}_{\mathrm{t}} \bar{\lambda}_{\mathrm{b}}=0.25 ; \Sigma_{\bar{\eta}}=0.5$ respectively.

The figure shows a monotonically linear decreasing behavior for small values of the dimensionless frequency to follow of an asymptotic region when the system the $\mathrm{Q}$ factor is independent of the dimensionless frequency. 


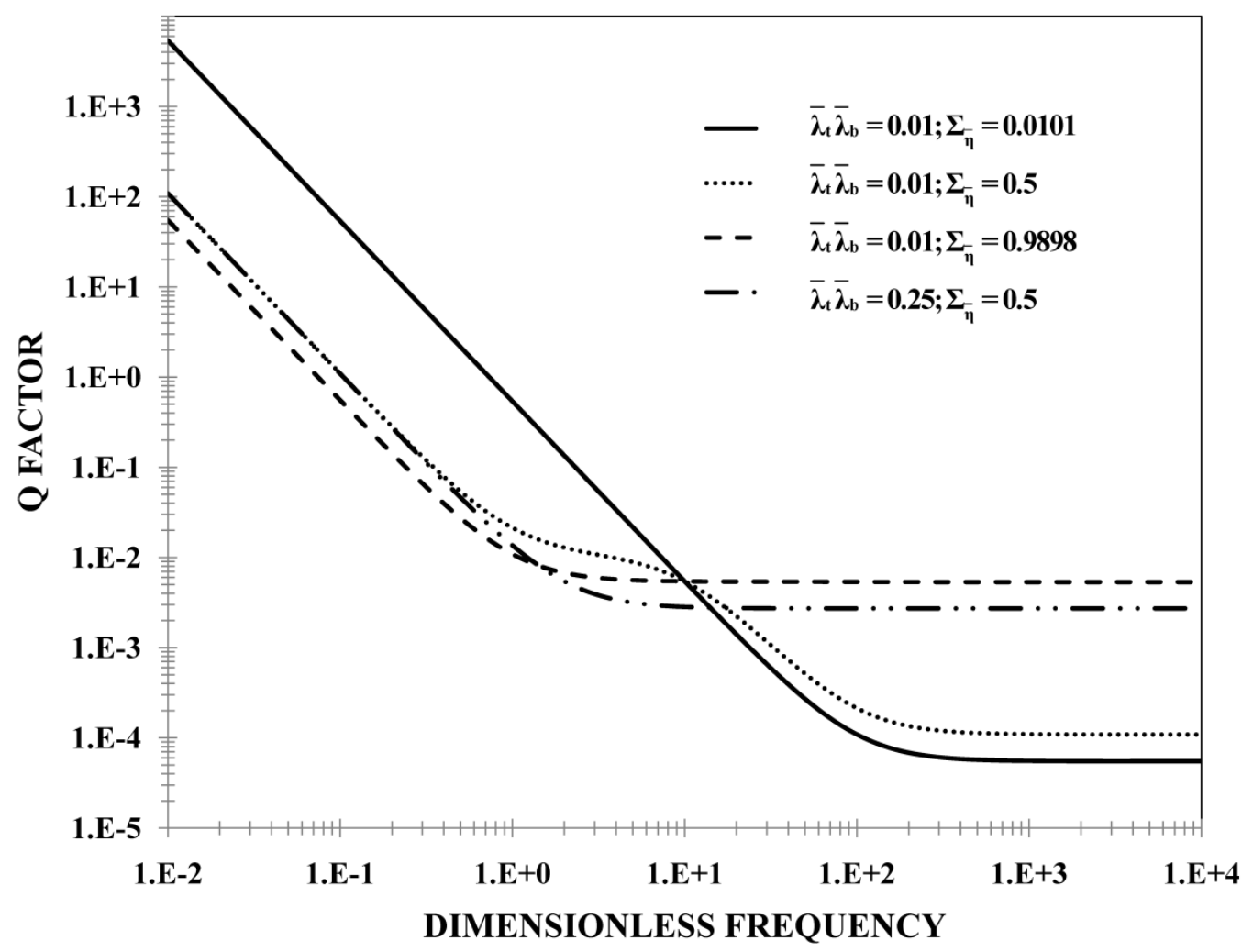

Figure $24-\mathrm{Q}$ factor as a function of the dimensionless frequency for different value of the dimensionless parametric dimensionless function $b_{1}^{*}$. The numerical values employed in the simulation are: a) $\bar{\lambda}_{\mathrm{t}} \bar{\lambda}_{\mathrm{b}}=0.01, \Sigma_{\bar{\eta}}=0.0101$; b) $\bar{\lambda}_{\mathrm{t}} \bar{\lambda}_{\mathrm{b}}=0.01, \Sigma_{\bar{\eta}}=0.9898$; c) $\bar{\lambda}_{\mathrm{t}} \bar{\lambda}_{\mathrm{b}}=0.25, \Sigma_{\bar{\eta}}=0.5$.

The minima value of the all plateaus is reached when the system presents low values of the memory (asymmetric case) and the total-bulk viscosity. When the viscosity increases, the value of the plateau is shifted to high values of the $Q$ factor. A similar case occurs for the symmetric case in the memory and intermediate value of the bulk-viscous number. It is important to mention that at low values of the dimensionless frequency, $Q$ in all cases is dominated by the elastic energy and for a critical value of the dimensionless frequency the power dissipation dominates over the elastic storage energy and the $\mathrm{Q}$ factor is independent of the dimensionless frequency. 


\subsubsection{Resonant Effects}

\subsubsection{Resonance power $\overline{\mathrm{P}}_{\text {res }}$ as a function of resonance frequency $\overline{\mathrm{W}}_{\text {res }}$}

Figure 25 shows the resonance power $\overline{\mathrm{P}}_{\text {res }}$ as a function of the dimensionless resonance frequency $\overline{\mathrm{W}}_{\text {res }}$ (see eqns. $(43,48)$ ). In the four cases of this simulation, the mathematical behavior is the same. The power exhibits a pulse profile. The peak value of the resonance power is determined by a coupling between the memory and bulk-viscous mechanism effects. Finally, for value close to the maxima stiffness, i.e., $\mathrm{k} \approx 1$, the power goes to zero sharply when $\overline{\mathrm{w}} \approx 10$. Notice that, we have two important effects in the resonance power due to the bulkviscous and elastic mechanism. Increasing the bulk-viscous mechanism $\Sigma_{\bar{\eta}}$ reduces the value of the peak resonance., whereas the increase of the memory $\bar{\lambda}_{t} \bar{\lambda}_{b}$ shifts the pulse to lower frequencies.

\subsubsection{Resonance elastic membrane energy $\overline{\mathrm{E}}_{\text {mres }}$ as a function of resonance frequency $\overline{\mathrm{W}}_{\text {res }}$}

Figure 26 shows the resonance membrane energy $\bar{E}_{\text {mres }}$ as a function of the resonance frequency $\overline{\mathrm{W}}_{\text {res }}$. The dimensionless numbers used in the simulation are the same as Figure 27. 


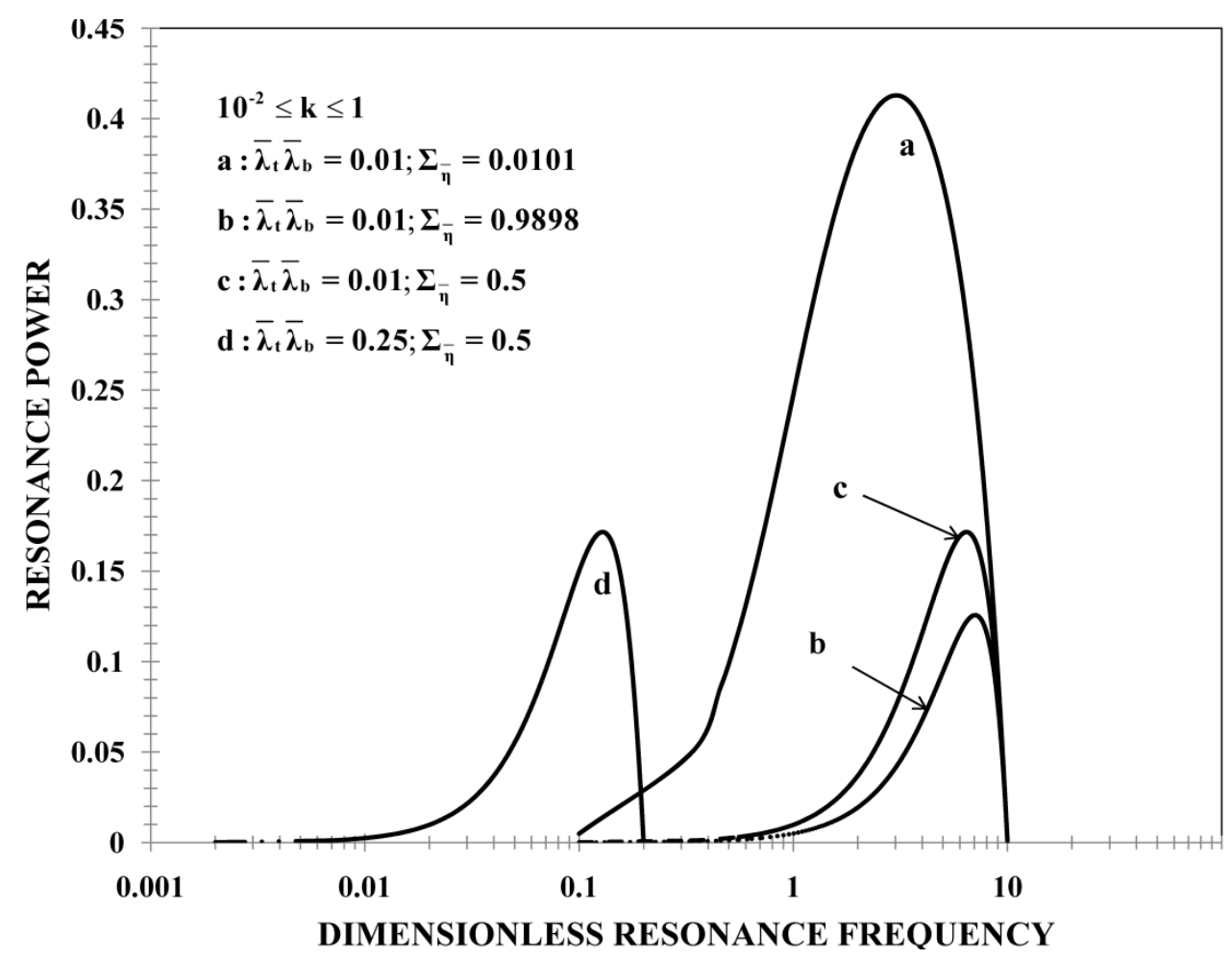

Figure 25 - Resonance power versus dimensionless frequency for different values of the dimensionless memory. The numerical values of the dimensionless numbers are: $10^{-2} \leq \mathrm{k} \leq 1$, a) $\bar{\lambda}_{\mathrm{t}} \bar{\lambda}_{\mathrm{b}}=0.1$, $\Sigma_{\bar{\eta}}=0.0101$; b) $\bar{\lambda}_{\mathrm{t}} \bar{\lambda}_{\mathrm{b}}=0.1, \Sigma_{\bar{\eta}}=0.9898$; c) $\bar{\lambda}_{\mathrm{t}} \bar{\lambda}_{\mathrm{b}}=0.1, \Sigma_{\bar{\eta}}=0.5$; d) $\bar{\lambda}_{\mathrm{t}} \bar{\lambda}_{\mathrm{b}}=0.25, \Sigma_{\bar{\eta}}=0.5$.

In the case of low memory and bulk viscous mechanism, the resonance membrane energy presents a decreasing behavior until a minima value to follow an increasing behavior (low value of the frequency) and a asymptotic behavior in the resonance membrane energy. In contrast, when the system shows a low memory and increase of the bulk-viscous mechanisms the elastic energy is zero for low values of the frequency, and for a critical value of the frequency, the elastic resonance energy increases to a resonance value close to $\overline{\mathrm{Em}}_{\text {res }}=0.5$. The same behavior is observed in the case of symmetric viscoelastic system and intermediate values of the bulk viscosity. 


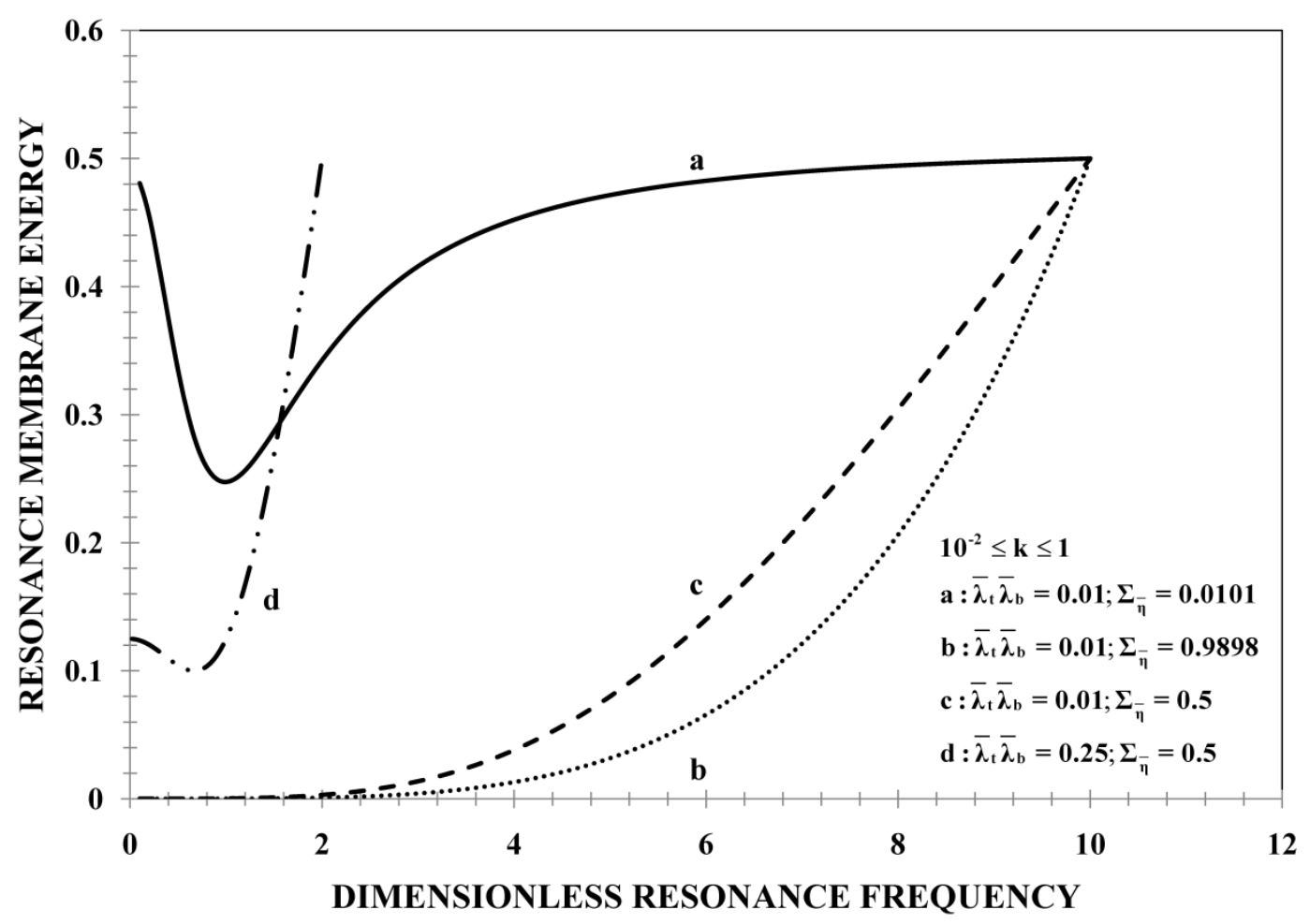

Figure 26 - Shows the resonance membrane energy versus dimensionless resonance frequency. The numerical values of the dimensionless numbers are: $10^{-2} \leq \mathrm{k} \leq 1$; a) $\bar{\lambda}_{\mathrm{t}} \bar{\lambda}_{\mathrm{b}}=0.1, \Sigma_{\bar{\eta}}=0.0101$; b) $\bar{\lambda}_{t} \bar{\lambda}_{b}=0.1, \Sigma_{\bar{\eta}}=0.9898$; c) $\bar{\lambda}_{t} \bar{\lambda}_{b}=0.1, \Sigma_{\bar{\eta}}=0.5$; d) $\bar{\lambda}_{t} \bar{\lambda}_{b}=0.25, \Sigma_{\bar{\eta}}=0.5$.

\subsubsection{Resonance power $\overline{\mathrm{P}}_{\text {res }}$ as a function of elastic ratio $k$}

Figure 27 shows the resonance power as a function of the elastic ratio for different values of the memory and bulk-viscous mechanisms . In all cases, the power shows a broad asymmetric downward parabolic profile. The critical elastic ratio value is shifted to the right as the total viscosity increases and also as the system symmetry (difference between the rheological characteristic of the fluids in contact with the membrane) is increased. The maximum value of $\overline{\mathrm{P}}_{\text {res }}$ increases with high asymmetry $\left(\bar{\lambda}_{t} \bar{\lambda}_{\mathrm{b}}<<1\right)$ and low total viscosity $\Sigma_{\bar{\eta}}$. 


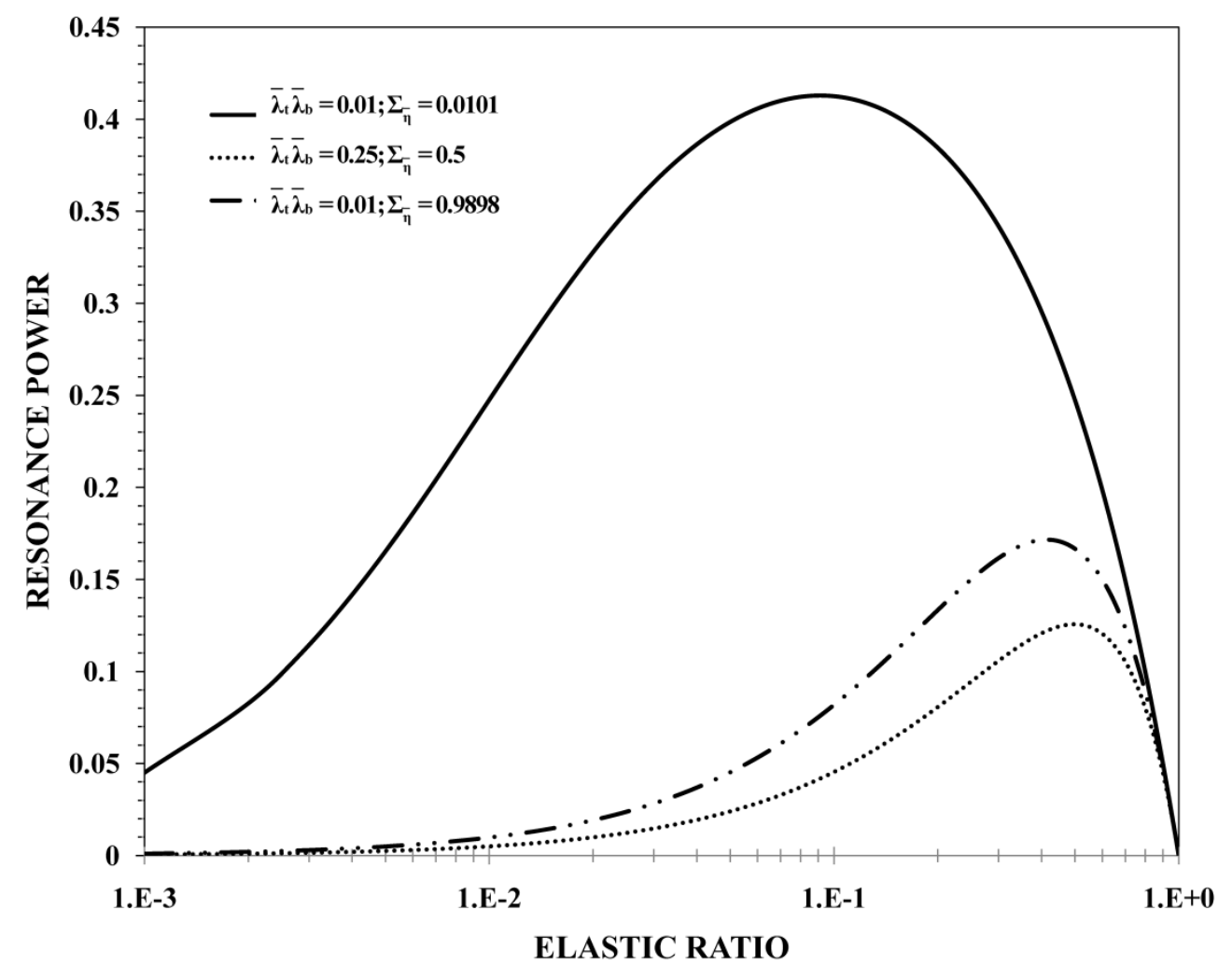

Figure 27 - Resonance power $\overline{\mathrm{P}}_{\text {res }}$ as a function of the elastic ratio $\mathrm{k}$ for different values of the memory $\bar{\lambda}_{t} \bar{\lambda}_{\mathrm{b}}$. The numerical values of the dimensionless numbers are: a) $\bar{\lambda}_{\mathrm{t}} \bar{\lambda}_{\mathrm{b}}=0.01, \Sigma_{\bar{\eta}}=0.0101$; b) $\bar{\lambda}_{\mathrm{t}} \bar{\lambda}_{\mathrm{b}}=0.01, \Sigma_{\bar{\eta}}=0.9898$; c) $\bar{\lambda}_{\mathrm{t}} \bar{\lambda}_{\mathrm{b}}=0.25, \Sigma_{\bar{\eta}}=0.5$.

\subsubsection{Resonance membrane energy $\bar{E}_{\text {mres }}$ as a function elastic ratio $k$}

Figure 28 shows the resonance elastic membrane energy $\overline{\mathrm{E}}_{\text {mres }}$ as a function of the elastic ratio $\mathrm{k}$ for different values of total viscosity $\Sigma_{\bar{\eta}}$ and memory $\bar{\lambda}_{\mathrm{t}} \bar{\lambda}_{\mathrm{b}}$. The values of the dimensionless numbers used in simulation are the same as in Figure 26. In contrast to power resonance, the elastic membrane energy is monotonically decreasing until a pronounced minimum value is reached (concave down). This curve is only observed when memory and bulk-viscous values are low. As total bulk viscosity increases we see the curve is monotonically increasing. There is an in-between behavior of the curve i.e. an initial plateau zone with a minimum value followed by monotonically increased behavior as the system approaches the symmetric case. 


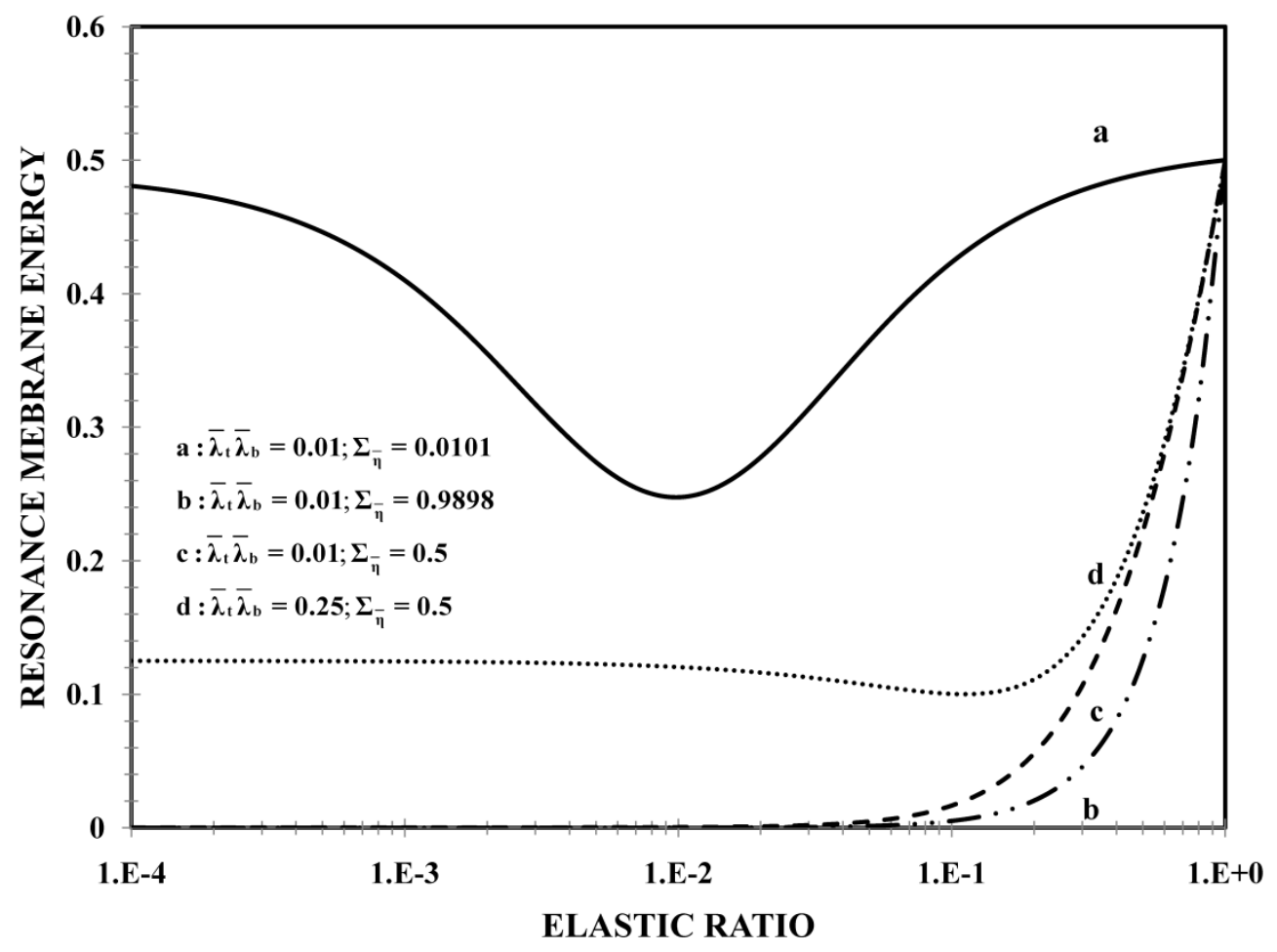

Figure 28 - Resonance energy $\overline{\mathrm{E}}_{\text {mres }}$ as a function of the elastic ratio $\mathrm{k}$ as a function for different values of the memory of the system $\bar{\lambda}_{\mathrm{t}} \bar{\lambda}_{\mathrm{b}}$. The numerical values of the dimensionless numbers are: a) $\bar{\lambda}_{\mathrm{t}} \bar{\lambda}_{\mathrm{b}}=0.1, \Sigma_{\bar{\eta}}=0.0101$; b) $\bar{\lambda}_{\mathrm{t}} \bar{\lambda}_{\mathrm{b}}=0.1, \Sigma_{\bar{\eta}}=0.9898$; c) $\bar{\lambda}_{\mathrm{t}} \bar{\lambda}_{\mathrm{b}}=0.1, \Sigma_{\bar{\eta}}=0.5$; d) $\bar{\lambda}_{\mathrm{t}} \bar{\lambda}_{\mathrm{b}}=0.25$, $\Sigma_{\bar{\eta}}=0.5$.

\subsubsection{Resonance $Q_{\text {res }}$ factor as a function of $k$}

Figure 29 shows the resonance $Q_{\text {res }}$ factor as a function of the elastic ratio $k$ for different values of the memory and total bulk-viscosity. When the total bulk-viscosity has a low value, i.e., $\Sigma_{\bar{\eta}}=0.0101$, the $\mathrm{Q}_{\text {res }}$ presents an almost constant behavior and its value is independent of the elastic ratio up to values of the elastic ratio close to 0.5 . When the elastic ratio goes to the unit $(k=1)$, the resonance $Q$ factor diverges. The second case is when the system shows a symmetric viscoelastic phases and the general behavior is similar to the low viscosity fluid, the only difference is in the value of the plateau, which is lower in this case. 


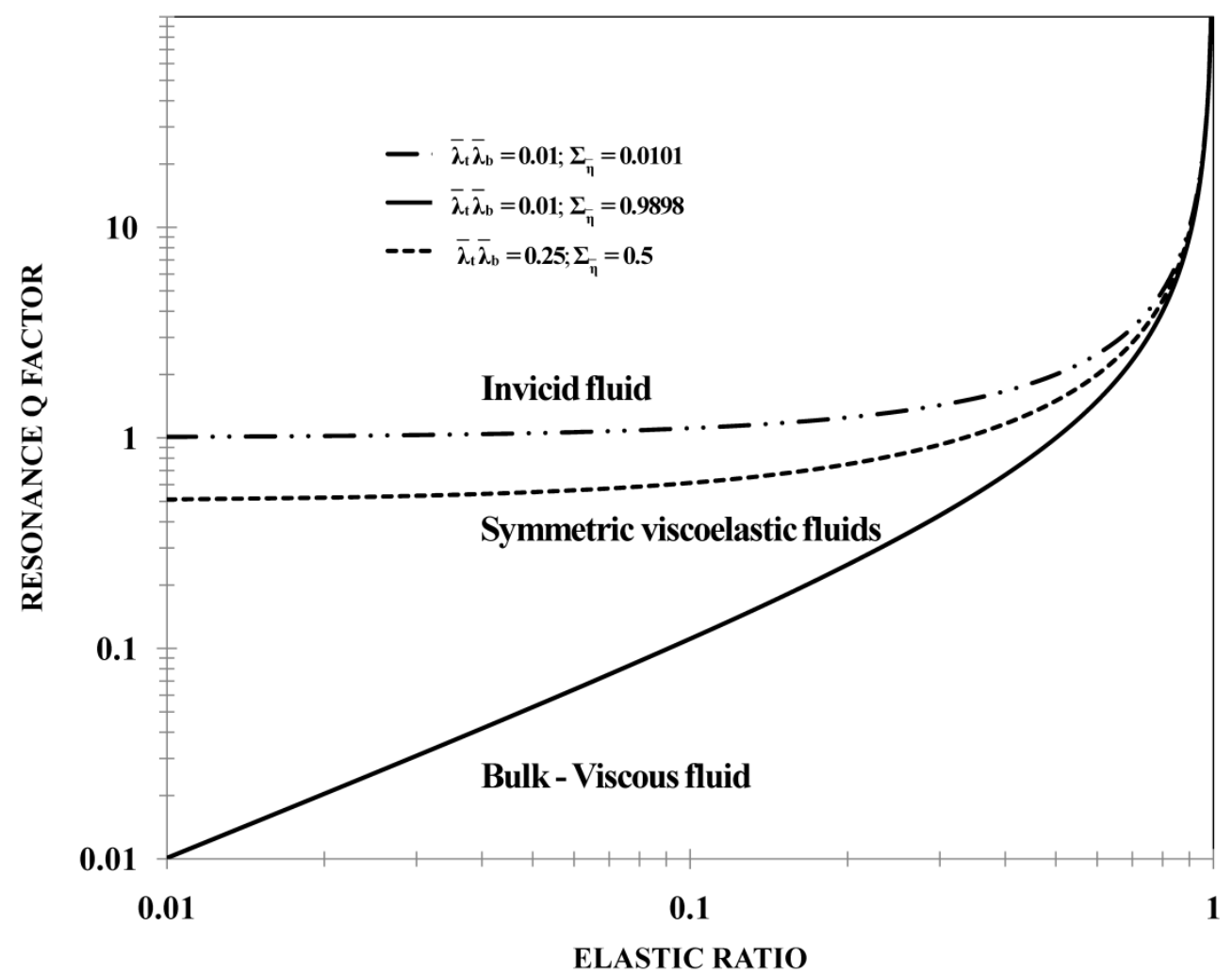

Figure 29 - Resonance $\mathrm{Q}_{\text {res }}$ factor as a function of the elastic ratio $k$ for different values of the memory. The values used in the numerical predictions are: (i) $\bar{\lambda}_{\mathrm{t}} \bar{\lambda}_{\mathrm{b}}=0.01 ; \Sigma_{\bar{\eta}}=0.01$; $\bar{\lambda}_{\mathrm{t}} \bar{\lambda}_{\mathrm{b}}=0.01 ; \Sigma_{\bar{\eta}}=0.9898$; (iii) $\bar{\lambda}_{\mathrm{t}} \bar{\lambda}_{\mathrm{b}}=0.01 ; \Sigma_{\bar{\eta}}=0.5$; (iv) $\bar{\lambda}_{\mathrm{t}} \bar{\lambda}_{\mathrm{b}}=0.25 ; \Sigma_{\bar{\eta}}=0.5$

\subsubsection{Viscoelastic/Membrane/Gas system}

One important particular case of the power dissipation is when the bulk-viscous mechanism is equal to the inverse of the elastic ratio, i.e., $b_{1}^{*} \cong 1 / k$. This implies that the bulk viscosity approaches to the maximum possible value, i.e. $\Sigma_{\bar{\eta}} \rightarrow 1$. In this case, the power dissipation has the following form:

$$
\overline{\mathrm{P}}\left[\overline{\mathrm{w}} ; \mathrm{b}_{1}^{*}=\frac{1}{\mathrm{k}}, \bar{\lambda}_{\mathrm{t}} \bar{\lambda}_{\mathrm{b}}, \mathrm{k}\right] \cong \mathrm{k} \frac{(1-\mathrm{k}) \overline{\mathrm{w}}^{2}}{\left(\mathrm{k}-\bar{\lambda}_{\mathrm{t}} \bar{\lambda}_{\mathrm{b}} \overline{\mathrm{w}}^{2}\right)^{2}+(\overline{\mathrm{w}})^{2}}
$$


At low dimensionless frequencies, the power contribution describes resonance behavior where the value of the peak is determined by the elastic ratio $\overline{\mathrm{P}}_{\text {peak }}=1-\mathrm{k}$. For high values of dimensionless frequency the power shows a decreasing monotonic behavior according to the following asymptotic equation: $\overline{\mathrm{P}} \cong\left(\mathrm{k}(1-\mathrm{k}) /\left(\bar{\lambda}_{\mathrm{t}} \bar{\lambda}_{\mathrm{b}}\right)^{2}\right) \overline{\mathrm{w}}^{-2}$. Physically the resonance effect is observed when one of the phases is an inelastic low viscous phase. In this case, the system has a material operation line (Figure 32), which is described by a hyperbolic function and, for any value of the curve $1 / \mathrm{k}$ the system presents a resonance behavior according to the equation (56). Figure 30 shows the dimensionless number $b_{1}^{*}=1 / k$ as a function of the elastic ratio $k$; the inset in Figure $\mathbf{3 0}$ shows several power curves. Notice that, the maximum of the resonance curve (inset) is reached for some critical $k$ value ( 0.50 in this case). There is a certain symmetry regarding the maximum in the power curve. Take for instance, $d(k=0.25)$ and e $(b=0.75)$ cases where the maximum value of the resonance curve is the same. However, there is a difference in the "width" of the curve, with wider curves for lower k values. The power maximum is related to the phases asymmetry (a very viscous liquid and a strongly viscoelastic liquid). 


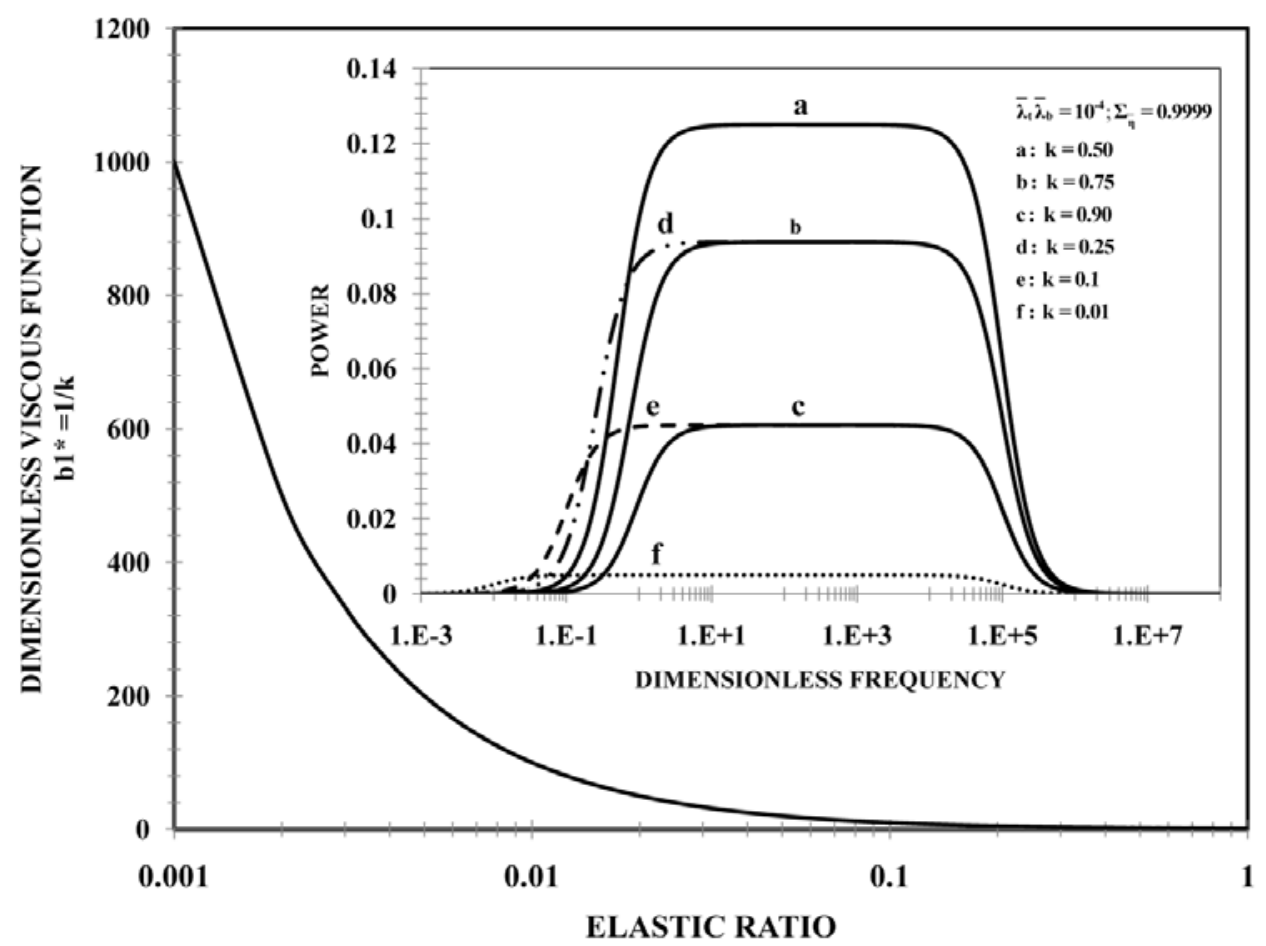

Figure 30 - Dimensionless bulk-viscous number as a function of the elastic ratio. In the inset the power dissipation as a function of the dimensionless frequency. The values used in the numerical predictions are: $\bar{\lambda}_{\mathrm{t}} \bar{\lambda}_{\mathrm{b}}=10^{-4} ; \Sigma_{\bar{\eta}}=0.9999$; (i) $\mathrm{k}=0.50$; (ii) $\mathrm{k}=0.75$; (iii) $\mathrm{k}=0.90$; (iv) $\mathrm{k}=0.99$.

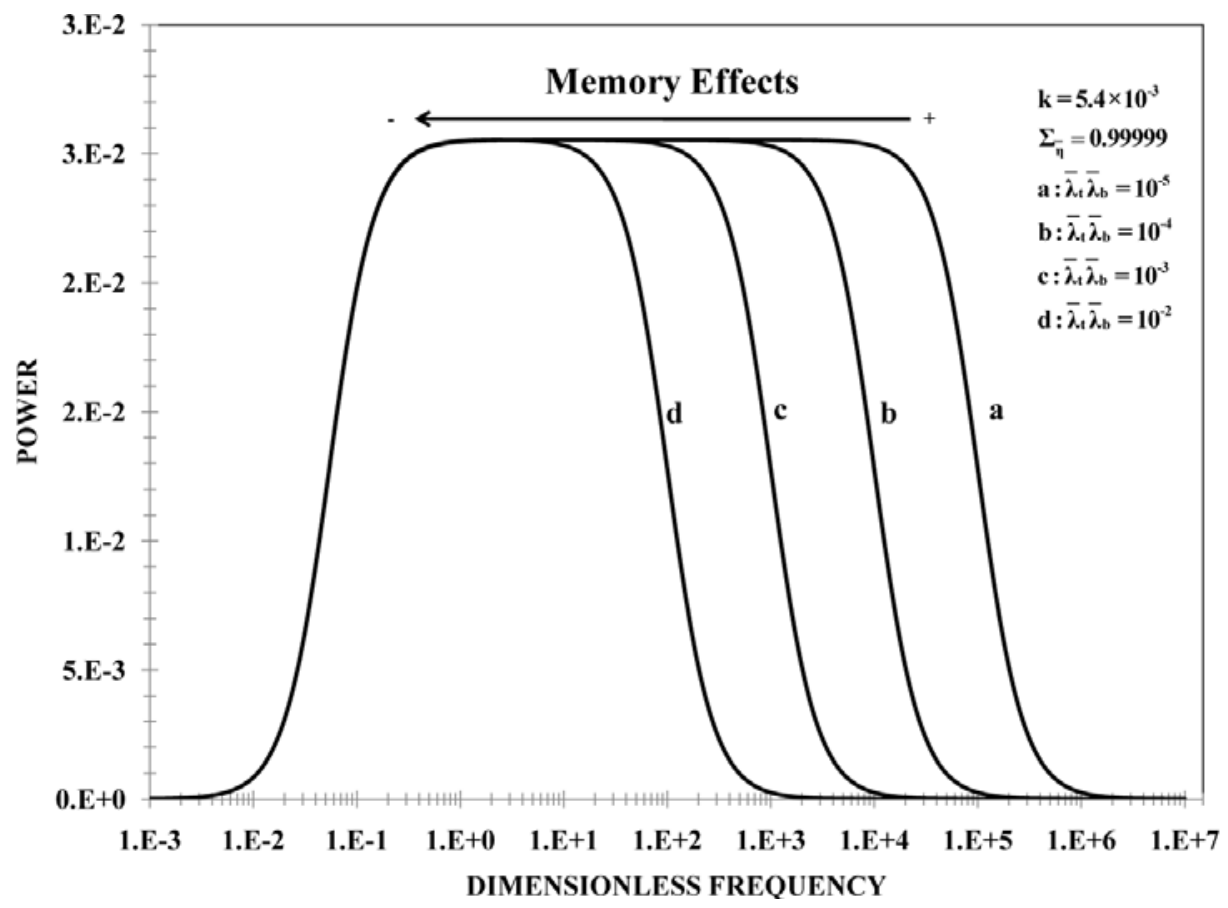

Figure 31 - Dimensionless $\overline{\mathrm{P}}$ as a function frequency. The values used in the numerical predictions are: $\bar{\lambda}_{\mathrm{t}} \bar{\lambda}_{\mathrm{b}}=10^{-4} ; \Sigma_{\bar{\eta}}=0.99999$; (i) $\mathrm{k}=0.50$; (ii) $\mathrm{k}=0.75$; (iii) $\mathrm{k}=0.90$; (iv) $\mathrm{k}=0.99$. 
Figure $\mathbf{3 1}$ shows the power dissipation $\overline{\mathrm{P}}$ as a function of dimensionless frequency, for increasing fluid symmetry $\bar{\lambda}_{\mathrm{t}} \bar{\lambda}_{\mathrm{b}}$. It is clear that the power dissipation plateau is the same in all the cases. In this case, the effect of the increasing memory is to narrow the band width and to shift the curve to the left.

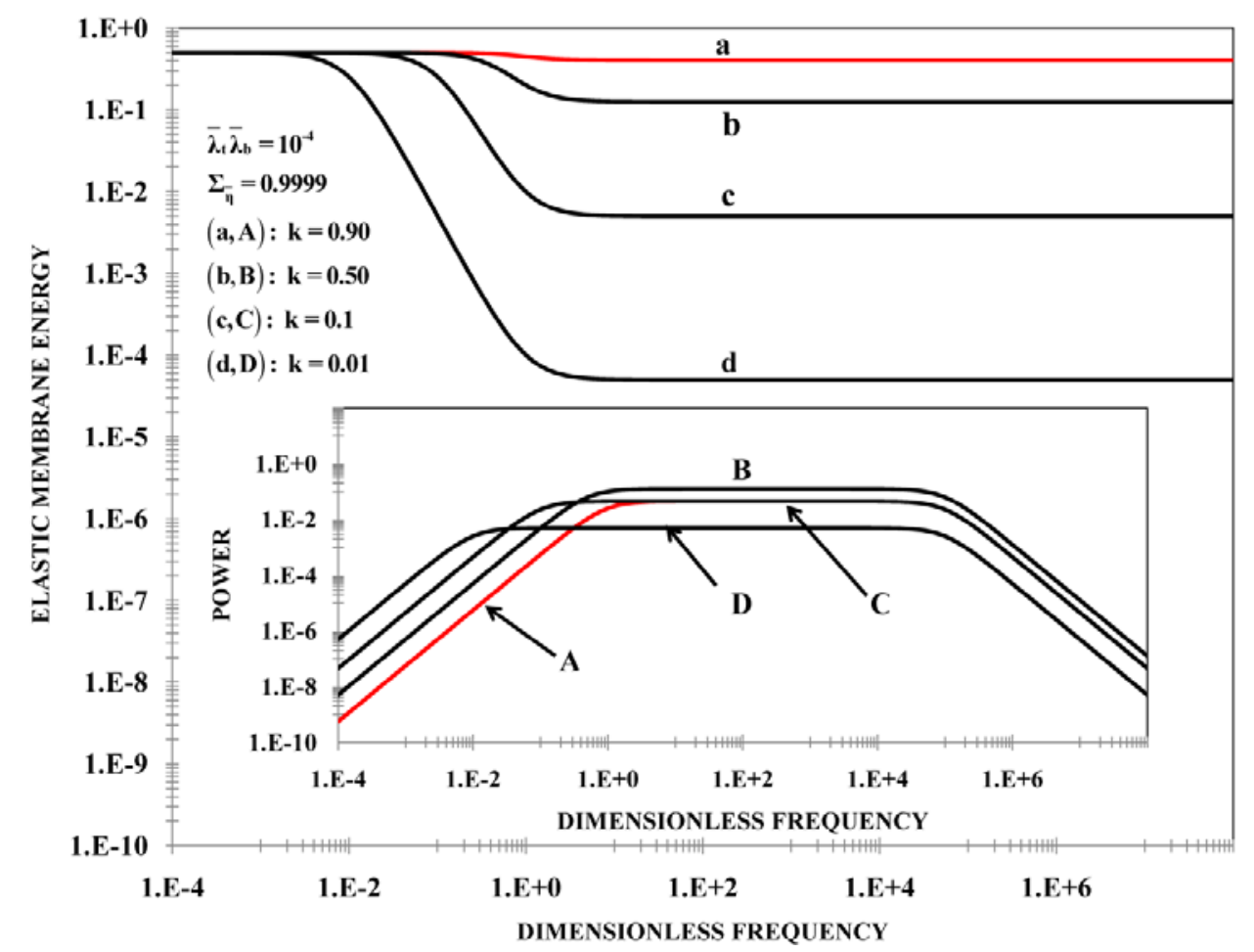

Figure 32 - Elastic membrane energy as a function of the dimensionless frequencies for different values of the elastic ratio. In the Inset, the power dissipation vs dimensionless frequency is showed. The parameters used in the simulation are the same of Figure 29.

Figure 32 shows the elastic membrane elastic energy and as a function of frequency. At low dimensionless frequencies, the elastic energy is independent of the frequency, for a critical value in the domain of the frequencies; the elastic energy shows a monotonically decreasing behavior (power law region) followed by a plateau at high frequencies. In contrast to the storage energy, the power dissipation shows a intermediate plateau region, where the power is 
independent of the dimensionless frequency, at low frequencies there is an increasing power law region and at high frequencies there is a decreasing power law region. For the cases $b$ and $B$, the dissipation and the storage energies are equal $(Q=1)$. For all other cases $Q \neq 1$, with the a,A case $Q>1$ and for the $c, C$ and $d, D$ cases $Q<1$. It is important to note, that the biological relevant case (sound amplification) is reached when $Q<1$ (maximum power), see Figure 33.

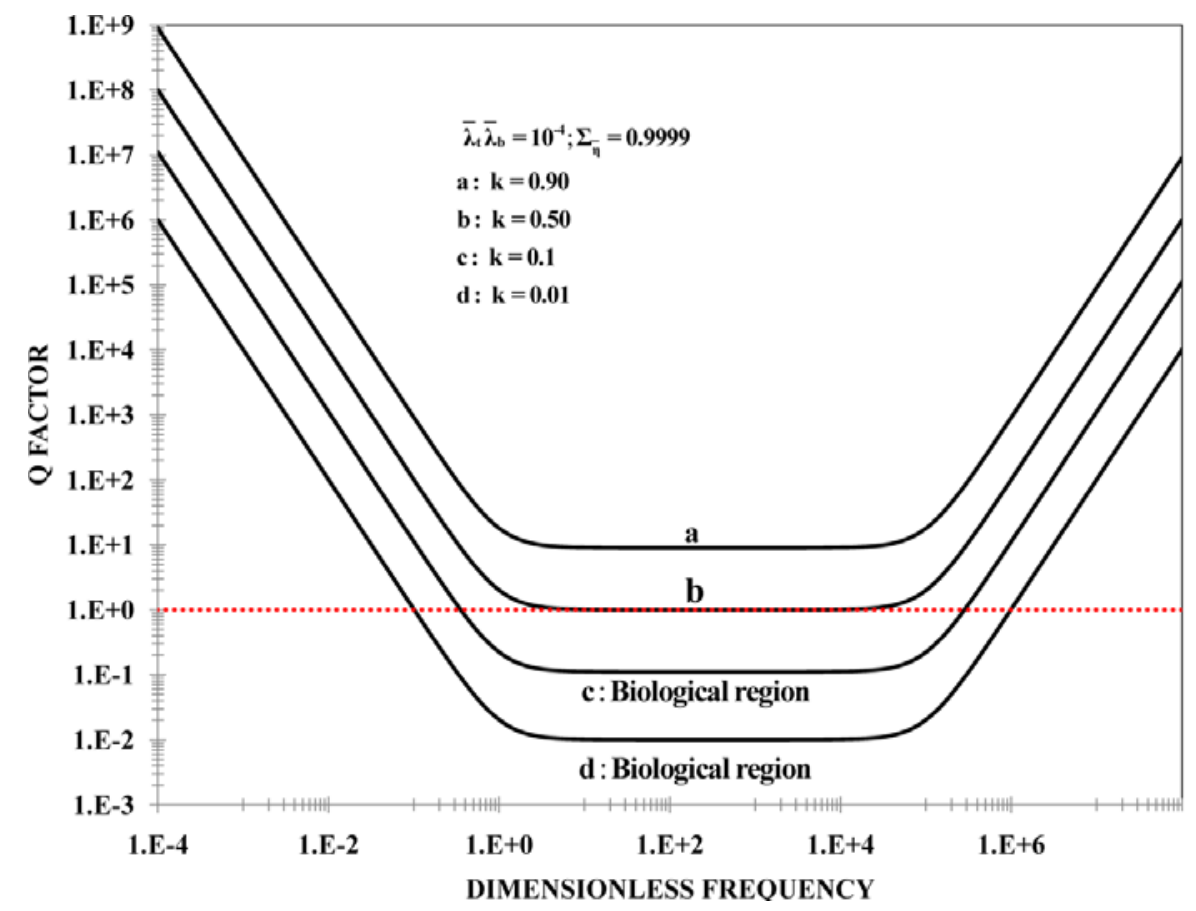

Figure $33-Q$ factor as a function of dimensionless frequency. The material properties used in the simulations are the same as Figure 30-32.

Combining the results from Figure 30-33, we can arrive at a qualitative pictures of power delivery $\overline{\mathrm{P}}$ and $\mathrm{Q}$ factor as a function of the elastic ratio (Figure 34). These qualitative figures show that the asymmetry of the phases, total bulk-viscosity and elastic ratio k play an important role in the system response. 


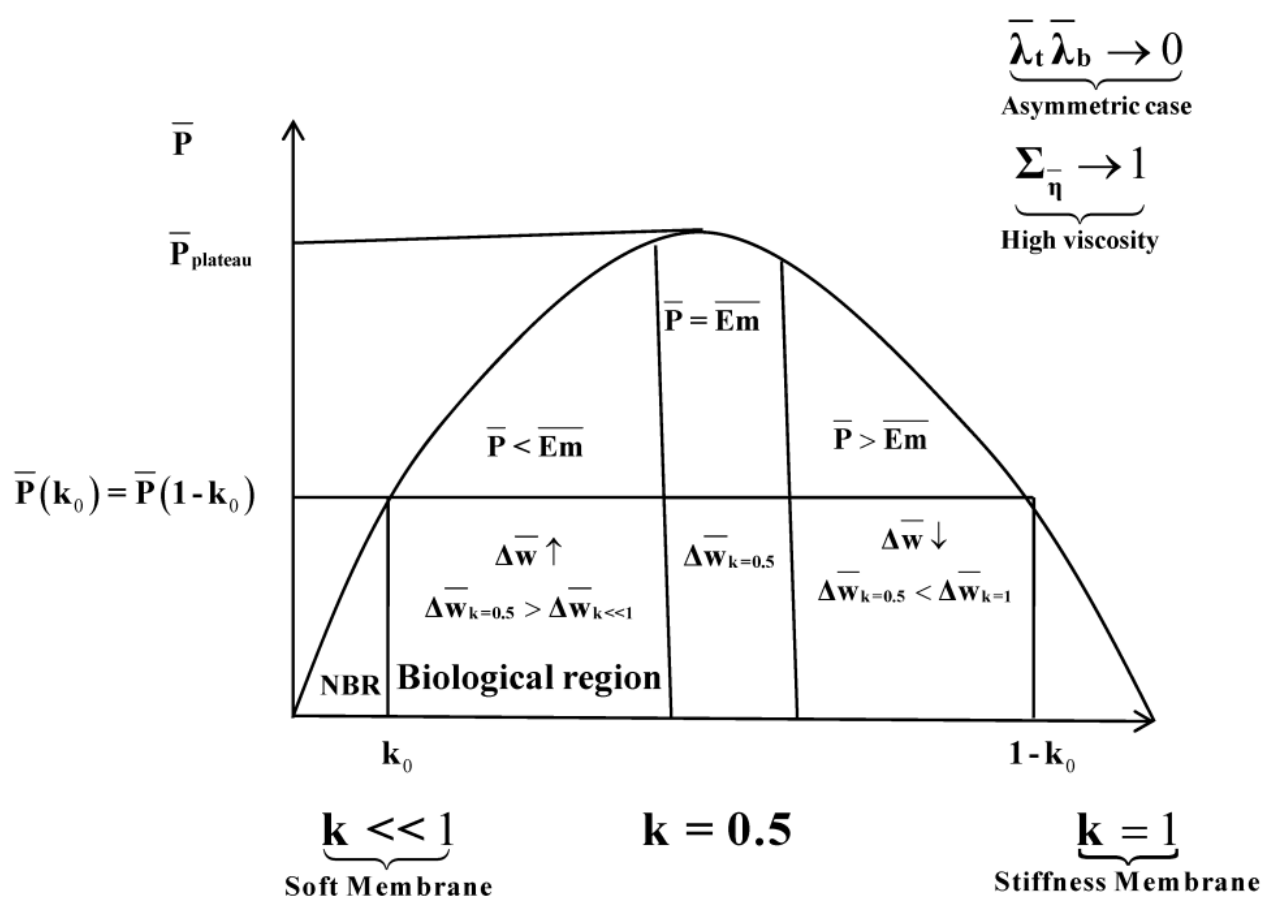

NBR : Non - biological region

a)

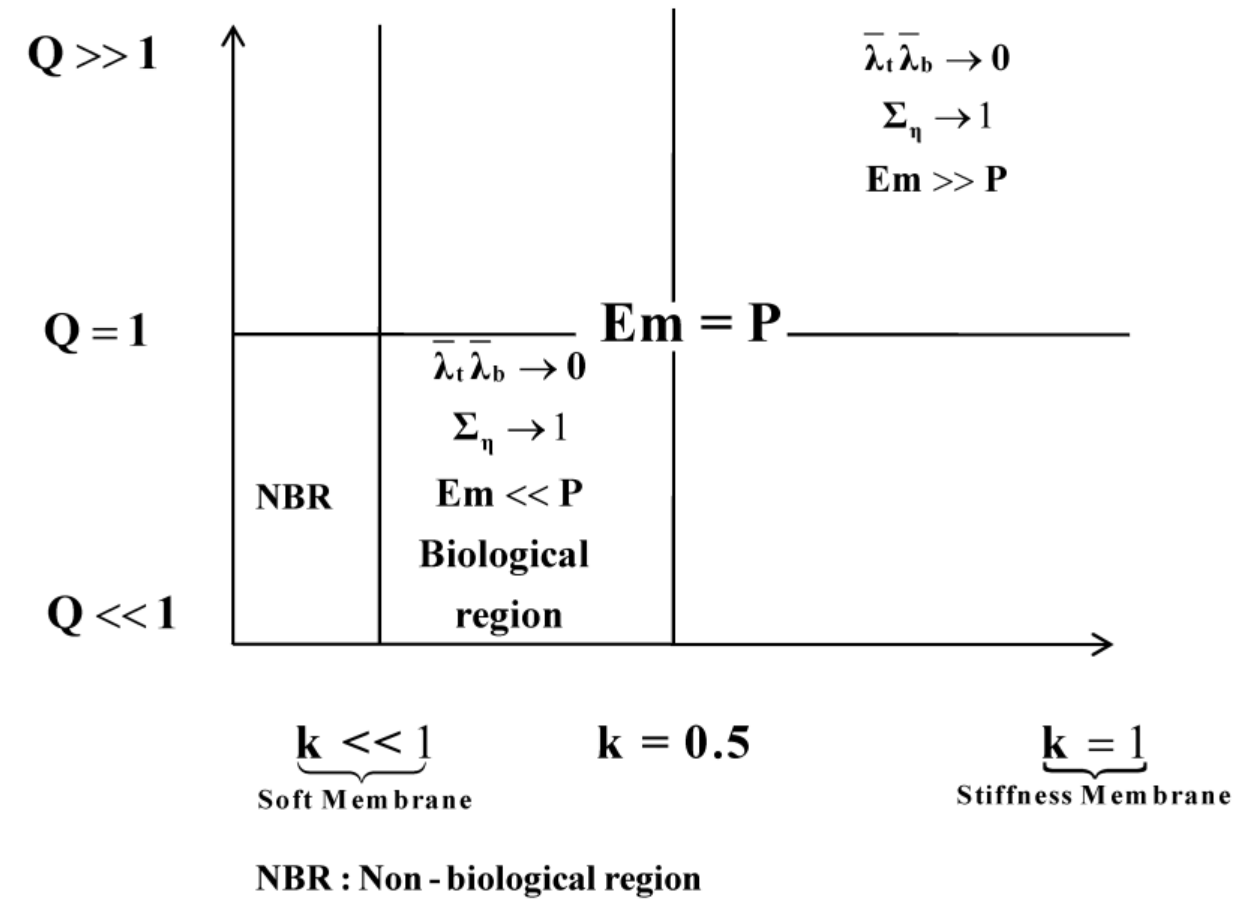

b)

Figure 34-Schematic representation of the power $\bar{P}$ and $Q$ factor as a function of the elastic ratio $k$. 


\subsection{Key Observations and Analysis}

Key observations based on Figure $\mathbf{3 4}$ regarding performance are:

\subsubsection{Power and Elastic Membrane Energy}

\section{a) Small elastic ratio: $k<0.5$}

(i) Low memory product (asymmetric case, $\bar{\lambda}_{\mathrm{t}} \bar{\lambda}_{\mathrm{b}}<<1$ ) and high viscosity $\left(\Sigma_{\bar{\eta}} \approx 1\right)$ lead to low power, high frequency width.

(ii) Power dominates over elastic storage

(iii) Biological relevant region $(Q<1)$ occurs for a specific interval of $\left(\mathrm{k}_{0}, \mathrm{k}\right)$

(iv) For $k<\mathrm{k}_{0}$, we have a non-operable membrane (Soft membrane)

\section{b) Intermediate elastic ratio $k=0.5$}

(v) Low memory (asymmetric case, $\bar{\lambda}_{\mathrm{t}} \bar{\lambda}_{\mathrm{b}}<<1$ ) and high viscosity $\left(\Sigma_{\bar{\eta}} \approx 1\right.$ ) lead to a power plateau, large frequency width in comparison to a small elastic ratio.

(vi) Power (dissipation) and elastic membrane energy (storage) values are equal.

\section{c) High elastic ratio $k->1$}

(vii) Low memory product (asymmetric case, $\bar{\lambda}_{\mathrm{t}} \bar{\lambda}_{\mathrm{b}}<<1$ ) high viscosity $\left(\Sigma_{\bar{\eta}} \approx 1\right.$ ) lead to small frequency width, low power

(viii) Elastic energy dominates over the power dissipation

(ix) Symmetry of the power exists for certain values of the elastic ratio, $\overline{\mathrm{P}}\left(\mathrm{k}_{0}\right)=\overline{\mathrm{P}}\left(1-\mathrm{k}_{0}\right)$ 


\subsubsection{Q-Factor}

\section{d) Small elastic ratio: $k<<1$}

(x) Low memory (asymmetric case) and high viscosity lead to low value of the $Q$ factor.

(xi) Biological zone where $Q<<1$ for a specific interval of $\left(\mathrm{k}_{0}, \mathrm{k}\right)$

(xii) For k lower than $\mathrm{k}_{0}$, the $Q$ factor is less than one, but the system does not present an operating biological zone ( too soft membrane).

(xiii) Biological region of the system occurs for a specific interval of $\left(\mathrm{k}_{0}, \mathrm{k}\right)$

(xiv) For less value of $\mathrm{k}_{0}$, we have an inoperable membrane (too soft membrane)

\section{e) Intermediate elastic ratio $k=0.5$}

(xv) Low memory product (asymmetric case, $\bar{\lambda}_{\mathrm{t}} \bar{\lambda}_{\mathrm{b}}<<1$ ) high viscosity $\left(\Sigma_{\bar{\eta}} \approx 1\right.$ ) lead to power plateau, large frequency width in comparison to small elastic ratio $k$.

(xvi) Power and elastic membrane energy (storage) values are equal

(xvii) Q factor is equal to one

\section{f) High elastic ratio $k->1$}

(xviii) Low memory product (asymmetric case, $\bar{\lambda}_{\mathrm{t}} \bar{\lambda}_{\mathrm{b}}<<1$ ) high viscosity $\left(\Sigma_{\bar{\eta}} \approx 1\right.$ ) lead to small frequency width, low power

(xix) Elastic energy dominates over the power dissipation

(xx) Q factor is greater than one. 


\subsection{Materials and Biological Applications}

\subsubsection{Outer Hair Cells: Biology and Physiology}

As reviewed by Sachs, Brownell, and Petrov [6], the OHC of the ear uses electromotility to overcome viscosity and thus sharpen the resonance effect to increase the required frequency resolution. Receptor potential generates active oscillations of the cell body in $\mathrm{OHC}$ [6]. The mechanical response to the electric input drives the oscillations in the cell's length which occur at the frequency of the incoming sound, providing mechanical amplification [6]. Rabbitt et al. [7] formulated a mathematical model for the OHC based in first principles and they analyzed the power conversion of the dissipation energy in the frequency space domain. Their model includes a mixture-composite constitutive model of the active lateral wall and spatially distributed electromechanical fields. Their research predicted the following:

a) The peak power efficiency is likely to be tuned to a specific frequency, dependent upon $\mathrm{OHC}$ length, and this tuning may contribute to the place principle and frequency selectivity in the cochlea.

b) OHC power output can be detuned/attenuated by increasing the basal conductance of the cell, a parameter likely controlled by the brain via the efferent system.

c) Power output efficiency is limited by mechanical properties of the load, suggesting impedance of the organ of Corti may be matched regionally to the $\mathrm{OHC}$.

d) The high power efficiency, tuning, and efferent control of outer hair cells are the direct result of biophysical properties of the cells, thus providing the physical basis for the remarkable sensitivity and selectivity of hearing. 
Figure 35 compares the dimensional power conversion efficiency, dimensionless power, elastic energy and $\mathrm{Q}$ factor as a function of the dimensional frequency and dimensionless frequency for Rabbit's model and the present models. The value of the maxima and minima power efficiency is due to a coupling between the OHC stiffness, fluid viscosity, entrained mass and $\mathrm{OHC}$ intracellular axial electrical resistance of the cell. All their results were done under control conditions of the impedance (low and high values). In this paper, the low values of the impedance are associated with the action of efferent neurotransmitter on the base of the OHC. The most efficient frequency depends upon cell length. Shorter cells show peak efficiencies at higher frequencies, while longer cell show peaks efficiencies at lower frequencies.

\subsubsection{Present Model}

In our flexoelectric model, the resonance power (dissipation), obeys a triple coupling effect: (i) asymmetric of the phases $\bar{\lambda}_{\mathrm{t}} \bar{\lambda}_{\mathrm{b}}<<1$, (ii) bulk-viscous mechanism $\left(\Sigma_{\bar{\eta}} \approx 1\right)$ and (iii) elastic ratio k. The width of the resonance power depends on the elastic ratio $k$ and $\bar{\lambda}_{\mathrm{t}} \bar{\lambda}_{\mathrm{b}}$. The elastic membrane energy presents two plateaus and an intermediate power law zone. The second plateau in the elastic energy is determined by the elastic ratio k. The power law zone and plateau of the elastic membrane energy and power dissipation are the most significant aspects from a biological and physiological standpoint in the $\mathrm{OHC}$ cells. Here, the value of the $\mathrm{Q}$ factor is less than one, which means the power dissipation dominates over the elastic energy and the system can behave as an amplifier. In our model, the biological zone $(Q<<1)$ is a consequence of the minimum and maximum elastic and power energy, which is regulated by the electro mechanical and physiological system through brain and efferent systems of the OHC. 


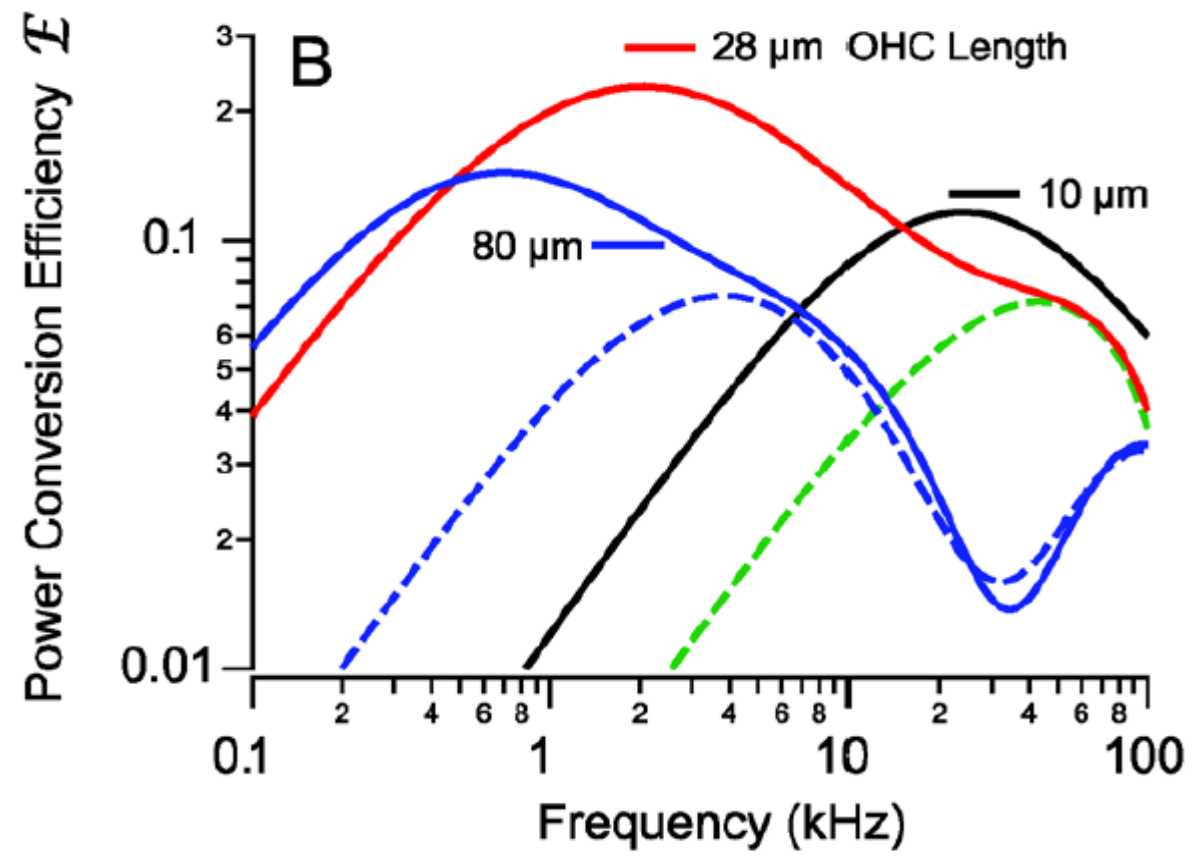

a) Rabbit's model

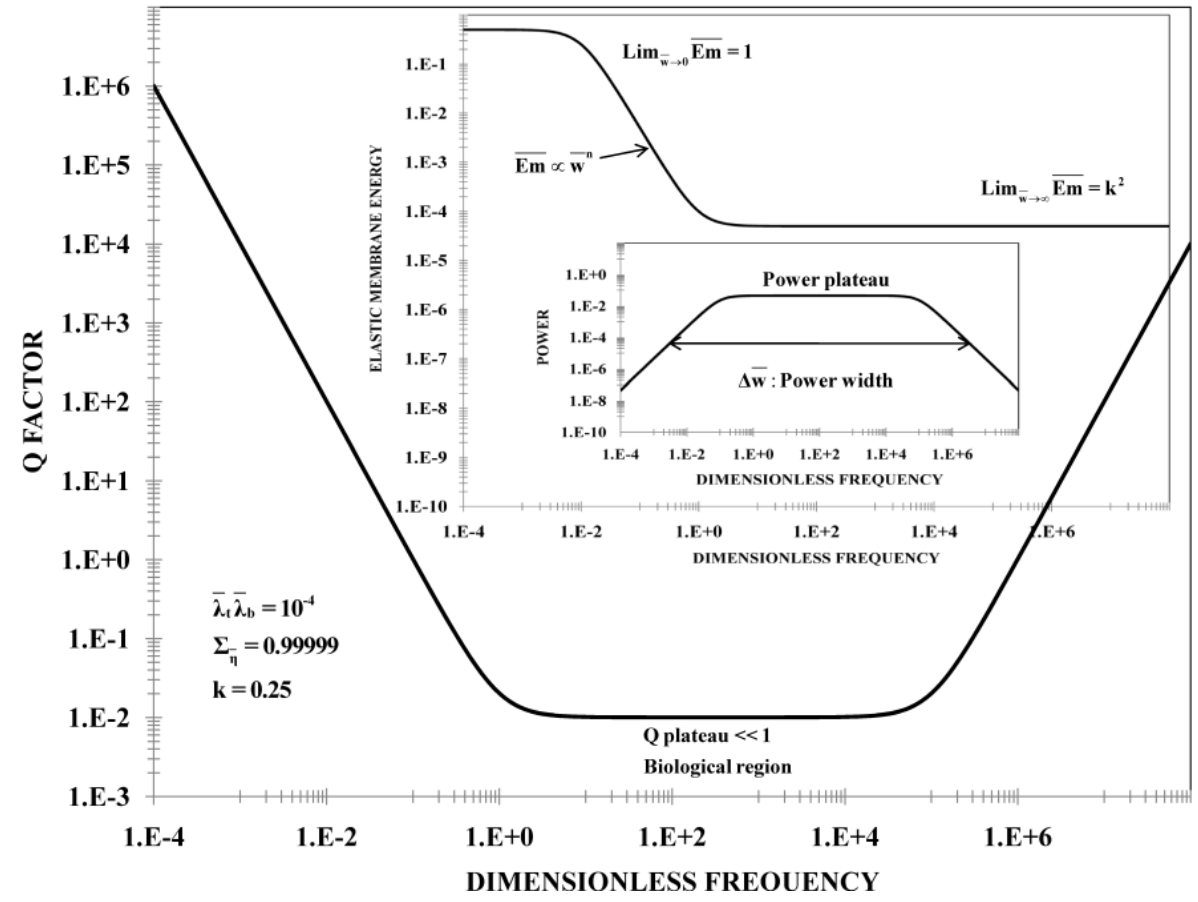

b) Present model

Figure 35 - Rabbitt's [7] model (a) and present model (b). 
In Table 1 a summary comparing Rabbit's and present models is shown.

Table 1:

Comparison between Rabbitt's and Present model

\begin{tabular}{|c|c|c|}
\hline & Rabbitt's model & Present Model \\
\hline System & $\mathrm{OHC}$ cells & $\begin{array}{l}\text { Flexoelectric membrane } \\
\text { embedded in two } \\
\text { Viscoelastic phases }\end{array}$ \\
\hline Mathematical model & Electromechanical & $\begin{array}{c}\text { Flexoelectric } \\
\text { And } \\
\text { Rheological }\end{array}$ \\
\hline Differential/Integral & Differential & Differential \\
\hline Constitutive equation & Newtonian & Viscoelastic \\
\hline Flow & Couette & Poiseuille flow \\
\hline $\begin{array}{c}\text { Power } \\
\text { Elastic energy } \\
\text { Q factor }\end{array}$ & $\begin{array}{c}\text { Analytical expression } \\
\text { NA } \\
\text { NA }\end{array}$ & $\begin{array}{l}\text { Analytical expression } \\
\text { Analytical expression } \\
\text { Analytical expression }\end{array}$ \\
\hline Peak Power & $\begin{array}{c}\text { Specific Frequency } \\
\text { OHC Length } \\
\text { Principle selective in } \mathrm{OHC}\end{array}$ & $\begin{array}{l}\text { Asymmetric Phases } \\
\text { Bulk-viscous } \\
\text { Elastic Ratio }\end{array}$ \\
\hline Minimum Power & $\begin{array}{c}\text { Specific Frequency } \\
\text { OHC Length } \\
\text { Principle selective in } \mathrm{OHC}\end{array}$ & NA \\
\hline
\end{tabular}


(Continued) Table 1:

Comparison between Rabbitt's [7] and Present model

\begin{tabular}{|c|c|c|}
\hline Minimum Power & $\begin{array}{c}\text { Specific Frequency } \\
\text { OHC Length } \\
\text { Principle selective in } \mathrm{OHC}\end{array}$ & NA \\
\hline $\begin{array}{l}\text { Peak-Power } \\
\text { physiology }\end{array}$ & $\begin{array}{c}\text { Brain } \\
\text { Efferent System } \\
\text { Biological properties of the } \\
\text { cell }\end{array}$ & $\begin{array}{c}\text { Brain } \\
\text { Efferent System } \\
\text { Blood }\end{array}$ \\
\hline $\begin{array}{l}\text { Resonance } \\
\text { frequency }\end{array}$ & $\begin{array}{c}\text { OHC Length } \\
\text { Principle selective in } \mathrm{OHC}\end{array}$ & $\begin{array}{c}\text { Asymmetric viscoelastic } \\
\text { phases } \\
\text { Elastic ratio }\end{array}$ \\
\hline Width power & OHC Length & $\begin{array}{c}\text { Asymmetric viscoelastic } \\
\text { phases } \\
\text { Elastic ratio }\end{array}$ \\
\hline Elastic energy & NA & $\begin{array}{c}\text { Asymmetric viscoelastic } \\
\text { phases } \\
\text { Bulk-viscous } \\
\text { Elastic ratio }\end{array}$ \\
\hline Biological region & $\begin{array}{c}\text { Specific Frequency } \\
\text { OHC Length } \\
\text { Principle selective in } \mathrm{OHC}\end{array}$ & $\begin{array}{c}\text { Asymmetric viscoelastic } \\
\text { phases } \\
\text { Bulk viscousity } \\
\text { Elastic ratio }\end{array}$ \\
\hline
\end{tabular}




\subsubsection{Dimensional Model}

\section{a) Power}

The present model, indeed inspired by the $\mathrm{OHC}$, shares similar properties as a physical membrane embedded in viscoelastic surroundings that also functions as a mechanical amplifier at characteristic (resonant) frequencies [40,63-69]. In this context, the dimensional power dissipation is given by the following expression:

$$
\mathrm{P}=\pi \mathrm{a}^{2} \mathrm{~L} \Sigma_{\mathrm{G}} \mathrm{M} \frac{\Sigma_{\eta} \mathrm{w}^{2}+\mathrm{X}_{\mathrm{G} \lambda} \lambda_{\mathrm{t}} \lambda_{\mathrm{b}} \mathrm{w}^{4}}{\left(\mathrm{M}-\left(\mathrm{M}+\Sigma_{\mathrm{G}}\right) \lambda_{\mathrm{t}} \lambda_{\mathrm{b}} \mathrm{w}^{2}\right)^{2}+\left(\mathrm{M} \Sigma_{\lambda}+\Sigma_{\eta}\right)^{2} \mathrm{w}^{2}}
$$

Equation (57) depends on the geometrical, elastic membrane and viscoelastic parameters, and for resonance frequency: $w_{\text {res }}=\sqrt{M /\left(M+\Sigma_{G}\right) \lambda_{t} \lambda_{b}}$, and high frequencies the following important results for the dissipation are obtained:

$$
\frac{\mathrm{P}_{\text {res }}}{\pi \mathrm{a}^{2} \mathrm{~L}}=\frac{\Sigma_{\mathrm{G}} \Sigma_{\eta} \mathrm{M}}{\left(\mathrm{M} \Sigma_{\lambda}+\Sigma_{\eta}\right)^{2}}+\frac{\Sigma_{\mathrm{G}} \mathrm{X}_{\mathrm{G} \lambda} \mathrm{M}^{2}}{\left(\mathrm{M} \Sigma_{\lambda}+\Sigma_{\eta}\right)^{2}\left(\mathrm{M}+\Sigma_{\mathrm{G}}\right)} ; \frac{\mathrm{P}_{\infty}}{\pi \mathrm{a}^{2} \mathrm{~L}}=\frac{\Sigma_{\mathrm{G}} \mathrm{M}}{\left(\mathrm{M}+\Sigma_{\mathrm{G}}\right)^{2}} \frac{\mathrm{X}_{\mathrm{G} \lambda}}{\lambda_{\mathrm{t}} \lambda_{\mathrm{b}}} \quad(58 \mathrm{a}, \mathrm{b})
$$

Neglecting elastic membrane contributions, we have:

$$
\mathrm{P}_{\text {res }}=\pi \mathrm{a}^{2} \mathrm{~L} \frac{\Sigma_{\mathrm{G}} \mathrm{M}}{\Sigma_{\eta}} ; \quad \mathrm{P}_{\infty}=\pi \mathrm{a}^{2} \mathrm{~L} \frac{\mathrm{MX}}{\lambda_{\mathrm{t}} \lambda_{\mathrm{b}} \Sigma_{\mathrm{G}}}
$$

Both equations show that the value of the resonance and terminal power are completely determined by a coupling between the geometrical, elastic-membrane and viscoelastic material properties. 


\section{b) Viscous/Solid/Viscoelastic phase}

When one of the two liquid phases has a small viscoelastic Maxwell number and small elasticity, equation (57) has the asymptotic form:

$$
\mathrm{P} \approx \pi \mathrm{a}^{2} \mathrm{~L} \Sigma_{\mathrm{G}} \mathrm{M} \frac{\eta_{\mathrm{t}} \mathrm{w}^{2}}{\left(\mathrm{M}-\left(\mathrm{M}+\Sigma_{\mathrm{G}}\right) \lambda_{\mathrm{t}} \lambda_{\mathrm{b}} \mathrm{w}^{2}\right)^{2}+\left(\mathrm{M} \lambda_{\mathrm{t}}+\eta_{\mathrm{t}}\right)^{2} \mathrm{w}^{2}}
$$

In particular, when the elastic membrane is equal to the total bulk viscosity, the above equation takes the following form:

$$
\mathrm{P}_{\mathrm{res}} \cong \frac{\pi \mathrm{a}^{2} \mathrm{~L}}{2} \frac{\mathrm{M}}{\lambda}=\frac{\pi \mathrm{a}^{2}}{4} \frac{1}{\lambda}\left(\gamma_{\mathrm{o}}+\left(\mathrm{k}_{\mathrm{c}}+\overline{\mathrm{k}}_{\mathrm{c}} / 2\right) \mathfrak{I}\right)
$$

This result means that the resonance power increases with the geometrical parameters of the capillary and elastic membrane whereas it decreases with the viscoelastic relaxation Maxwell time (memory). Equation (61) represents the resonance power contribution for two identical viscoelastic fluid systems. One important application of this equation is the direct characterization of the elasticity of the membrane. Once the power resonance is tuned, the biological membrane elasticity is computed directly from the above expression, and the rest of the material and geometrical parameters are easily obtained from rheometric experiments (Oscillatory measurements using cone and plate geometries for the Maxwell relaxation time) and the radius and capillary length from metrology experiments. 


\subsubsection{Material Properties}

The material properties play an important role in the power dissipation, storage elastic energy and $Q$ factor. In this work we focus only when $Q$ factor is less that the unite, i..e., $Q<<1$. The following important issues are summarized:

a) $Q<<1$ is reached for low value of the elastic ratio, i.e., $\mathrm{k}<0.5$ (Soft Membrane); small memory number (viscous/ solid/viscoelastic phases) and higher value of the total bulk viscosity in the system. It clear that all these coupled mechanisms are responsible to get the condition $\mathrm{Q}<<1$.

b) This parameter describes a material point in the 3D material phase diagram. This material point lives in the planes Inertia Membrane and Bulk-Viscous-Membrane. The Inertia Bulk-Viscous plane is not possible in our system since our primary hypothesis is that the membrane elasticity is different from zero.

\subsubsection{Optimization of Material Properties}

The material properties that leads to the optimal power generation are :

a) Small elastic ratio $\mathrm{k}<<1$, meaning that the membrane elasticity is much smaller than the fluid elasticity. Experimentally, the soft membrane must be in contact with at least a high molecular weight viscoelastic solution.

b) Small memory number. It is important to note that the memory number is related to the asymmetric viscoelastic characteristic of the contacting phases. The optimal material 
state is: viscous fluid/soft membrane/ viscoelastic fluid. The corresponding optimal bulk fluids can be obtained by contacting a viscous liquid and a viscoelastic polymer solution.

c) Total bulk viscosity needs to be sufficiently high, for example by using viscosity modifiers.

d) There is an inherent symmetry in terms of $k$ : if $k=1-A$, with $A<<1$, the for $k=1-A$ is the same as for $k=A$. The key point is that even if power is almost equal, the elastic membrane energy is not. Hence, $Q$ optimal follows one $k$ and not the other, this means that $\mathrm{k}$ and $1-\mathrm{k}$ give the same power dissipation but not the same elastic membrane energy. To minimize $E$ and minimize $Q$, we use 1-k.

e) Biologically the dimensionless elastic ratio k cannot be zero, since we do have a soft membrane. There is a minima $\mathrm{k}$ below which functioning is not possible due to a weak membrane would not be able to maintain the fluid interface. Hence $k$ is bounded from below due to requirements of the membrane integrity.

f) The membrane tension can be modified adding different bio-molecules (proteins, carbohydrates). 


\subsection{Summary and Discussion}

\section{Summary}

The present viscoelastic model developed in the present work explains the amplitude, the peak frequency, the width of power plateau, elastic energy and $Q$ factor profile. The material parameters of importance are: (i) elastic membrane, (ii) Bulk-elasticity of the two viscoelastic phases, and (iii) Maxwell relaxation times.

- The specific ways to adapt these parameters are by changing the concentration and the molecular weight distribution.

- To increase power amplitude, one of the liquid phases must be weakly elastic and the other one completely viscoelastic (phase asymmetry).

- To shift the location of the power plateau and width of the power plateau, the elasticity of the membrane with respect to the bulk (viscoelastic phases) must be tuned.

- To widen the power plateau, the Maxwell relaxation times, elasticity of the membrane and viscoelastic phases must be modified.

Finally, with all these effects, it is clear that the optimum membrane performance can be shifted accordingly to operate at a desired frequency with only tuning the parameters analyzed which in practice could represent a real challenge for experimentalists. Additionally, we are exploring higher order models to characterize the high impedance (active mechanism) behavior of the $\mathrm{OHC}$ (solid line above-right). A 'double peak' similar to that seen in the high $\mathrm{Z}_{\mathrm{b}}$ case is observed when we introduce a third-order 'retardation effect' into our model. 


\section{Discussion}

Membrane flexoelectricity is a novel electromechanical coupling effect that occurs in polarizable media under geometric curvature. The sensor effect is performed by bending induced electric polarization, whereas the converse actuation effect is performed by the membrane curvature induced by an imposed electric field. Membrane flexoelectricity is relevant to the functioning of the Outer Hair Cells $(\mathrm{OHC})$ which act as amplifiers to counteract viscous dissipation through mechanic transduction and thus allowing hearing. The key challenge is to understand the coupling of oscillatory flexoelectric actuation and the viscoelastic phenomena of the fluids that are in contact with the oscillating membrane. An efficient method to describe membrane flexoelectricity is to use the liquid crystal analogy that follows by identifying the director field of a nematic with the unit normal to the membrane. A key parameter is the flexoelectric coefficient, oo the order $3-20 \mathrm{pC} / \mathrm{m}$ for biological membranes.

In this chapter we deal with the dynamics of the actuation flexoelectric mode. An integrated dynamical model for the average curvature of flexoelectric membranes oscillating in viscoelastic fluid media under capillary confinement was formulated using a previously presented shape equation based on the liquid crystal approach $[14,16,18]$. The membrane curvature dynamics is given by a balance between the viscoelastic stress jump from the contacting bulk liquids, the restoring membrane effective tension, and the driving flexoelectric force. Using the flexoelectric shape equation in conjunction with a viscoelastic capillary flow model for the contacting phases we obtained a new average curvature dynamic equation. Perhaps surprisingly, the equation is shown to map into the classical solids Burger model, where the stress $\boldsymbol{\sigma}$ and strain $\boldsymbol{\varepsilon}$ become the electrical field $\mathbf{E}$ and curvature of the membrane 
H respectively [50-57]. The second order model for the electric field input and curvature output is given by a balance of inertial, viscous, and elastic effects, originating from the flexoelectric membrane and the viscoelastic fluids. A material phase diagram that defines the nature of the material coefficients and the two possible regimes under small oscillatory electric fields is developed. Using dimensionless variables, the physics of the system can be described with three dimensionless numbers which are associated to the asymmetric of the phases, total bull-viscous and elastic ratio mechanism.

It is found that the dimensionless number associated to the asymmetry of the viscoelastic phases (memory) controls the maxima and minima values of the bulk-viscous mechanisms. For a simple harmonic function (sinusoidal form) of the input electrical field, the average curvature can be separated into out-in phase and in-out of phase curvature moduli contributions. Analytical expressions for the power dissipation, elastic storage moduli and $\mathrm{Q}$ factor were obtained (elastic membrane energy / power dissipation) from the curvature moduli.

The frequency response of membrane average curvature and output power is characterized by the presence and absence of inertia (inelastic fluids). The frequency response is characteristic of a second order dynamical system with second order input and displays a single resonant peak in the in-out of phase curvature moduli and presents two plateaus at high and low frequencies and an intermediate power law behavior for the out-in phase curvature moduli respectively $\left\{\overline{\mathrm{H}}_{\mathrm{io}}, \overline{\mathrm{H}}_{\mathrm{oi}}\right\}$. The value of the resonance frequency depends on the asymmetry of the phase through the product between the Maxwell relaxation times (bulk fluid memory).

The elastic membrane energy $\overline{\mathrm{E}}_{m}$ presents two plateaus a low and high value of the resonance frequencies with an intermediate power law behavior. In contrast, the power 
dissipation P displays a linear behavior for small frequencies, and a monotonically increasing behavior for moderate and high dimensionless frequencies.

The effect of increasing the total viscosity in the system shifts the curves of the elasticenergy and power to higher values of the dimensionless frequency. The resonance effect is entirely dominated by the value of the dimensionless product between the viscoelastic times. The elastic resonance ratio is a measure of the bulk-viscous and inertial mechanism in the system and represents a phase diagram for: (i) bulk-viscous, (ii) inviscid fluid, (iii) symmetric viscoelastic fluids and (iv) Asymmetric fluids respectively. For values of the elastic ratio close to one, the elastic energy is independent of the frequency and the power displays a linear behaviour with the frequency.

When the viscous parametric function $b_{1}^{*}=1 / k$ a resonance power curve is obtained. The maximum value of the power plateau is reached for $k=0.5$. When the elastic ratio $k$ is less than 0.5 and close to 1 , the power plateau decreases drastically and the width of the curve also decreases for $k<0.5$ and increases for $k->1$. In addition, when the asymmetric of the phases decreases, the width of the power curve narrows.

Regarding the $Q$ factor, we found three important limits: (i) $Q>1$, (ii) $Q=1$, (iii) $Q<1$ ( biological relevant state) . It was found that for a small elastic ratio, high asymmetry of the phases and a high value of the bulk-viscosity, the power dissipation dominates over the elastic energy $(Q<<1)$. It is important to note that the material properties of the biological viscoelastic liquids can be modified by addition of polymers the concentration of and molecular weight of added polymers, and solution $\mathrm{pH}$. 
The power dissipation and elastic membrane storage energy are relevant to the functioning of outer hair cells and they are dependent on the inertia emerging from the contacting viscoelastic phases together with the bulk-viscous and elastic ratio mechanisms. The predicted results shown in Figure $\mathbf{3 5}$ are consistent with biological performance.

One important future application of the present work could be the inverse problem, i.e. instead of giving the theoretical predictions we could start by obtaining material experimental parameter and adjust the power accordingly; this quantitative study will be pursued in the future (Equation 57).

Finally, viscoelastic flexoelectricity is a novel coupling mechanism that incorporates nonNewtonian flow, membrane mechanics, and electric polarization to transform electric power into mechanical power, of great current interest to human hearing and actuation research. 


\section{Chapter 4}

\section{Conclusion}

Membrane flexolectricity is a curvature-driven actuation mechanism of significant relevance in the human hearing processes, through the coupling between electric polarization created by sound waves, membrane elasticity driven by curvature oscillations, and viscoelastic flow in the surrounding contacting media. A fundamental understanding of the functioning of the Outer Hair Cells requires the integration of physiology, engineering, and material science. In this thesis, theory and simulation based on biological engineering and material science principles was formulated, developed, analyzed, characterized, and partially validated.

In our second order electromechanical model an electric field is related to the curvature of a flexoelectric membrane. This model is given by a balance between inertial, viscous, and elastic components. Since the objective of our investigation is to understand flexoelectric phenomena such as Outer Hair Cell electromotility, the novelty of the approach was to couple flexoelectric actuation with viscoelastic fluids in contact with an oscillating membrane. Equations and parameters are all analytical and numbers and values empirically based. Resonant and profile power dissipation, elastic storage and $\mathrm{Q}$ factor were investigated and results showed encouraging agreement with the literature.

One of our most important results is that the frequency response of curvature and power in our system is governed by inertia. The value of the resonance frequency depends on the asymmetry of surrounding fluid elasticity. This suggests that a bending LC membrane can 
achieve resonance only in certain material "beds". By optimizing the properties of the embedding material, membrane performance can be tuned to operate in particular ways at desired frequencies.

Material properties of biological tissues and liquids are not always constant - such as membrane stiffness. An accomplishment of our research is that the flexibility of our model aids in understanding system behaviour and expanding the limits of our current knowledge. In ongoing work, higher order models are being explored to fully characterize the OHC.

In sensor mode, a bending membrane would generate electric potentials. Our research thus far has focused on studying the actuating potential of flexoelectric membranes. Another important element for future research is to study this inverse phenomenon. Not only can we reverse the phenomenon of study but we can also reverse the approach to our research, as mentioned in Section 3.7, by finding experimental material parameters and calibrating our model accordingly. For this reason, a complete quantitative study is required for full validation.

In summary, membrane flexoelectricity is a versatile material property of quasi twodimensional materials, that has, as shown in this thesis, significant potential to explain physiological sensor/actuator mechanisms in hearing, as well as in future biomimetic applications including energy harvesting, artificial muscle, mechanical valves and more. 


\section{Bibliography}

[1] P.G.de Gennes, J. Prost, The physics of Liquid Crystals Second Edition, Oxford University Press, United Kingdom,1994.

[2] A. G. Petrov, Electricity and mechanics of biomembrane systems: Flexoelectricity in living membranes, Anal. Chim. Acta 568 (2006) 70-83.

[3] A. G. Petrov, Flexoelectricity of model and living membranes, BBA-Biomembranes 1561 (2001) 1-25.

[4] A. G. Petrov, Lyotropic state of matter, Gordon and Breach Science Publisher, Amsterdan, 1999.

[5] R. B. Meyer, Piezoelectric Effects in Liquid Crystals, Phys. Rev. Lett. 22 (1969) 918-921.

[6] F. Sachs, W.E. Brownell, A.G. Petrov, Membrane electromechanics in biology with a focus on hearing, MRS Bull. 34 (2009) 1-14.

[7] R.D. Rabbitt et al. and W.E. Brownell, Power Efficiency of Outer Hair Cell Electromotility, PloS, 2009.

[8] J.S. Patel, R.B. Meyer, First description of flexoelectrooptics in uniform lying helix alignment, Phys. Rev. Lett. 58 (1987) 1538-1540.

[9] B.J. Broughton, M.J. Clarke, A. E. Blatch, H.J. Coles, Optimized flexoelectric response in achiral liquidcrystal phase device, J. Appl. Phys. 98 (2005) 034109/1-034109/6.

[10] G. Carbone, P. Salter, S. Elston, P. Raynes, L.D. Sio, S. Ferjani , G. Strangi, C. Umeton, R. Bartolino, Appl. Phys. Lett. 95 (2009) 011102/1-011102-3.

[11] P.S. Salter, S. J. Elston, E. P. Raynes, L. A. Parry-Jones, Alignment of the Uniform Lying Helix Structure in Cholesteric Liquid Crystals, Jpn. J. Appl. Phys. 48 (2009) 101302/1-101302/5.

[12] A.I. Jakli. J.E. Harden, S. Sprunt, J.T. Gleeson, U.S. Patent No.0182026 (2010).

[13] M. Kleman, O. D. Lavrentovich, Soft Matter Physics: An Introduction, Springer Verlag, New York, 2003. 
[14] A.D. Rey, Nonlinear actuator model for flexoelectric membranes, Int. J. of Design Nature Ecodynam. 3 (2008) 28-38.

[15] A .D. Rey, "Liquid crystals model of membrane flexoelectricity", Phys. Rev. E 74 (2006) 011710/1011710/11.

[16] A. D. Rey, Liquid crystals model of membrane flexoelectricity, Virtual J. Biol. Phys. Res. 12 (2006) 011710/1-011710/11.

[17] A. D. Rey, Polar fluid model of viscoelastic membranes and interfaces, J. Coll. Int. Sci. 304 (2006), 226-238.

[18] A.D. Rey, Linear Viscoelastic Model for Bending and Torsional Modes in Fluid Membranes, Rheol. Acta 47 (2008) 861-871.

[19] P.A. Kralchevsky, K. Nagayama, Particles at fluids interfaces and membranes, Elsevier, Amsterdam, 2001.

[20] A.D. Rey, Mechanics of soft solids-liquid crystals interfaces, Phys. Rev. E 72 (2005) 011706101170616.

[21] A.D. Rey, Capillary models for liquid crystal fibers, membranes, films, and drops, Soft Matter 2 (2007) 1349-1368.

[22] A.D. Rey, Liquid crystals models of biological materials and processes, Soft Matter 6 (2010) 34023429.

[23] A.D. Rey, E. E. Herrera-Valencia, Liquid crystal models of biological materials and silk spinning, Biopolymers (2011) doi: 10.1002/bip.21723

[24] A.D. Rey, E. E. Herrera-Valencia, Micromechanics model of liquid crystals anisotropic triple lines with applications to contact line self-assembly, Langmuir 26 (2010) 1349-1368.

[25] A.D. Rey, E. E. Herrera Valencia, A model for mesophase wetting thresholds of sheets, fibers and fiber bundles, Soft Matter 7 (2011) 5002-5009. 
[26] J. Harden, R. Teeling, J.T. Gleeson, S. Sprunt, A. Jákli, “Converse flexoelectricity effect in a bent-core nematic liquid crystal", Phys. Rev. E 78 (2008) 031702/1-031702/5.

[27] J. Harden, M. Chambers, R. Verduzco, P. Luchette, J.T. Gleeson, S. Sprunt, A. Jákli, Giant flexoelectricity in bent-core nematic liquid crystals elastomers, Appl. Phys. Lett. 96 (2010) 102907/1-102907/3.

[28] R. M. Raphael, A. S. Popel, W. E. Brownell, Recent Developments in Auditory Mechanics, World Scientific Publishing, Singapore,1993.

[29] W.E. Brownell, Evoked mechanical responses of isolated cochlear outer hair cells, Science 227 (1985) 194-196.

[30] B. C. J. Moore, Cochlear Hearing Loss: Physiological and Technical Issues, second ed., John-Wiley \& Sons Ltd, England, 2007.

[31] R. D. Hawkins, M. Lovett, The developmental genetics of auditory hair cells, Hum. Mol. Genet. 13 (2004) R289-R296.

[32] A.A. Spector, N. Deo, J.T. Ratnanather, R.M. Raphael, Electromechanical models of the outer hair cell composite membrane, J. Membr. Biol. 209 (2006) 135-152.

[33] U. Yucel, J. Y. Kazakia, Viscoelastic effects in pressure transduction, J. Non-Newtonian Fluid Mech. 123 (2004) 59-66.

[34] R.M. Raphael, A. S. Popel, W. E. Brownell, A membrane bending model of outer hair cell electromotility, Biophys. J. 78 (2000) 2844-2862.

[35] R. D. Rabbits, S. Clifford, K. D. Breneman, B. Farrell, W.E. Brownell, Power efficiency of outer hair cell somatic electromotility, Comput. Biol. 5 (2009) 1-14.

[36] Illustration from The Encyclopedia of Science taken with permission; http://www.daviddarling.info.

[37] J. Ashmore, Cochlear outer hair cell motility. Physiol Rev. 2008 Jan;88(1):173-210. 
[38] M. Kelly, Regulation of cell fate in the sensory epithelia of the inner ear, Nat. Rev. Neuro. Sci. (7) 837-849 (2006).

[39] D.T. Kemp, Stimulated acoustic emissions from within the human auditory system, J. Acoust. Soc. Am. 64 (1978).

[40] J.S. Oghalai, H.B. Zhao, J.W. Kutz, W.E. Brownell, Voltage-and tension-dependent lipid mobility in the outer hair cell plasma membranes, Science 287, (2000) 658.

[41] W.E. Brownell, A.A. Spector, R.M. Raphael, and A.S. Popel. Micro- and nanomechanics of the cochlear outer hair cell wall. Annu. Rev. Biomed. Eng., 2001, 3, 169-194.

[42] I.A. Belyantseva, H.J. Adler, R. Curi, G. I. Frolenkov, and B. Kachar. Expression and Localization of Prestin.. during Development of Electromotility in Cochlear OHCs. J. Neuroscience, 2000, 20.

[43] P. Dallos et. al., Presin-based OHC motility is necessary for mammalian cochlear amplification. Nueron. 2008 58(3):333-9.

[44] W.E. Brownell et. al., Effects of prestin on Membrane mechanics and Electromechanics. Eng. Med. Bio. Soc. 2007.

[45] R.A. Eatock, K.M. Hurley, M.A. Vollrath. Mechanoelectrical and voltage-gated ion channels in mammalian vestibular hair cells. Audiol. Neuootol. 2002 7(1):31-5.

[46] R.B. Bird, R.C. Armstrong, O. Hassager, Dynamics of polymeric liquids, Vol. 1, John Wiley \& Sons, New York, 1987.

[47]H. Barnes, K. Walters, An introduction to rheology, H. A. Barnes, J. F. Hutton, K. Walters, Elsevier, Amsterdan, 1989.

[48] F. Calderas, A. Sánchez-Solis, A. Maciel, O. Manero, The transient flow of the PETPENMontmorillonite clay Nanocomposite, Macromol Symp. MACROMEX 283- 284 (2009) 354-360.

[49] E.E. Herrera, F. Calderas, A.E. Chavez, O. Manero, B. Mena, "Effect of random longitudinal vibrations pipe on the Poiseuille flow of a complex liquid", Rheol. Acta 48 (2009) 779-800. 
[50] E.E. Herrera, F. Calderas, A.E. Chavez, O. Manero, Study on the pulsating flow of worm-like micellar solution, J. Non-Newtonian Fluid Mech. 165 (2010) 174-183.

[51] J.C. Slattery, L. Sagis, E.S. Oh, Interfacial transport phenomena, $2^{\text {nd }}$ ed., Springer, New York, 2007.

[52] D.A. Edwards, H. Brenner, D.T. Wasan, Interfacial transport processes and rheology, ButterworthHeinemann, Burlington MA, 1991.

[53] R. Quintanilla, K.R. Rajagopal, On Burgers fluids, Math. Meth. Appl. Sci. 29 (2006) 2133-2147.

[54] A. Jager, R. Lackner, Identification of viscoelastic model parameters by means of cyclic nanoindentation testing, Int. J. Mat. Res. 99 (2008) 1-8.

[55] W. N. Findley, J. S. Lai, K. Onaran, Creep and relaxation of non-linear viscoelastic materials with an introduction to linear viscoelasticity, Dover Publications, New York, 1976.

[56] M. Qausar, Attenuation properties of viscoelastic material, Pure Appl. Geophys. 131 (1989) 703-713.

[57] R.F. Cooper, Seismic Wave Attenuation: Energy Dissipation in Viscoelastic Crystalline Solids, Rev Mineral Geochem. 51 (2002) 253-290.

[58] S. T. Nguyen, L. Dormieux, Y. L. Pape, J. Sanahuja, A burger model for the effective behavior of a microcracked viscoelastic solid, Int. J. Damage Mech. (2011) doi: 10.11177/1056789510395554

[59] M. Fikus, P. Pawlowski, Bioelectrorheolgical model of the cell. 2. Analysis of creep and its experimental verification, J. Theor. Biol. 137 (1989) 365-373.

[60] N. N. Mohsenin, Physical properties of plant and animal materials: structure, physical characteristic, and mechanical properties, second ed., Gordon and Beach, New-York, 1986

[61] I.G. Main, Vibrations and waves in physics, 3d edition, Cambridge University Press, Cambridge, 1993.

[62] D.J. Panagopoulos, A. Karabarbounis, L. H. Margaritis, Mechanism for action of electromagnetic fields on cells, Biochem. Biophys. Res. Commun. 298 (2002) 95-102.

[63] D.J. Panagopoulos, N. Messini, A. Karabarbounis, A.L. Philippetis, L. H. Margaritis , A mechanism for action of oscillating electric fields on cells, Biochem. Biophys. Res. Commun. 272 (2000) 634-640. 
[64] F.Sachs, W. E. Brownell, A.G. Petrov, Membrane Electromechanics in Biology, MRS 2009.

[65] N. Messini, A. Karabarbounis, A.L. Philippetis, L. H. Margaritis, A mechanism for action of oscillating electric field on cells, Biophys. Res. Commun. 272 (2000) 634-640.

[66] A.R. Thornton, P.J. Abbas, Low-frequency hearing loss: Perception of filtered speech psychophysical tuning curves, and masking, J Acoust Soc Am. 67 (1980) 638-643.

[67] D. Ehrenstein, K.H. Iwasa, Viscoelastic relaxation in the membrane of the auditory outer hair cell, J. Biophys. 71 (1996) 1087-1094.

[68] J.F. Greenleaf, M. Fatemi, M. Insana, Selected methods for imagine elastic properties of biological tissues, Annual Review of Biomedical Engineering.5 (2003) 57-78.

[69] A. Manduca, T.E. et. al., Magentic resonance elastography: Noninvasive mapping of tissue elasticity, Medical Image Analysis 5 (2001) 237-254. 


\section{Appendices}

\section{Appendix A: Preliminary Differential Geometry}

This Appendix summarizes the differential geometry used in the paper $(1,6,25)$. Consider an interface whose points are locate in 3D space by a position vector $\mathbf{R}$, given parametrically by:

$\mathbf{R}=\mathbf{R}\left(\mathrm{u}^{\alpha}\right), \alpha=1,2$

The surface coordinates induce two tangential base vectors $\mathbf{a}_{\alpha}$ defined by:

$\mathbf{a}_{\alpha}=\frac{\partial \mathbf{R}}{\partial \mathbf{u}^{\alpha}}, \alpha=1,2$

The surface metric tensor $\mathbf{a}_{\alpha \beta}$ is defined by:

$\mathbf{a}_{\alpha \beta}=\mathbf{a}_{\alpha} \cdot \mathbf{a}_{\beta} ; \alpha, \beta=1,2$

whose determinant is

$\mathrm{a}=\operatorname{det}\left|\mathbf{a}_{\alpha \beta}\right|>0$

The corresponding reciprocal base vectors $\mathbf{a}^{\alpha}$ and metric tensor are:

$\mathbf{a}^{\alpha}=\frac{\partial \mathbf{u}^{\alpha}}{\partial \mathbf{R}}, \mathbf{a}^{\alpha \beta}=\mathbf{a}^{\alpha} \cdot \mathbf{a}^{\beta} ; \alpha, \beta=1,2$

The base and reciprocal base vectors define the surface unit tensor $\delta_{\alpha}^{\beta}$, and the dyadic surface idem factor $\mathbf{I}_{\mathrm{s}}$ :

$\mathbf{a}_{\alpha} \cdot \mathbf{a}^{\beta}=\delta_{\alpha}^{\beta}, \quad \mathbf{I}_{\mathrm{s}}=\mathbf{a}_{\beta} \mathbf{a}^{\alpha} \delta_{\alpha}^{\beta}=\mathbf{a}_{\alpha} \mathbf{a}^{\beta} \delta_{\beta}^{\alpha}=\mathrm{a}_{\alpha \beta} \mathbf{a}^{\alpha} \mathbf{a}^{\beta}=\mathrm{a}^{\alpha \beta} \mathbf{a}_{\alpha} \mathbf{a}_{\beta}$ 
where $\mathrm{a}^{\alpha \beta} \mathrm{a}_{\beta \gamma}=\delta_{\gamma}^{\alpha}$. The counterclockwise rotation of a vector around the unit normal $\mathbf{k}$ is given by the dyadic surface unit alternator $\varepsilon_{\mathrm{S}}$ :

$\varepsilon_{\mathrm{S}}=-\mathbf{k} \times \mathbf{I}_{\mathrm{S}}=-\mathbf{I}_{\mathrm{S}} \times \mathbf{k}=-\mathbf{k} \times \mathbf{I}=-\mathbf{I} \times \mathbf{k}=\mathbf{a}^{\alpha} \mathbf{a}^{\beta} \varepsilon_{\alpha \beta}=\mathbf{a}_{\alpha} \mathbf{a}_{\beta} \varepsilon^{\alpha \beta}$

where $\varepsilon_{\gamma \delta}=\mathrm{a}_{\alpha \gamma} \mathrm{a}_{\beta \delta} \varepsilon^{\alpha \beta}$. The surface unit normal $\mathbf{k}$ is given by:

$\mathbf{k}=\frac{1}{2} \boldsymbol{\varepsilon}_{\mathrm{s}}: \boldsymbol{\varepsilon}=\frac{1}{2} \boldsymbol{\varepsilon}^{\alpha \beta} \mathbf{a}_{\alpha} \times \mathbf{a}_{\beta}=\frac{1}{2 \sqrt{\mathrm{a}}}\left(\mathbf{a}_{1} \times \mathbf{a}_{2}-\mathbf{a}_{2} \times \mathbf{a}_{1}\right)$

where $\boldsymbol{\varepsilon}$ is the triadic spatial unit alternator. Other useful relations involving the surface unit normal $\mathbf{k}$ are:

$\mathbf{a}_{\alpha} \times \mathbf{a}_{\beta}=\mathbf{k} \varepsilon_{\alpha \beta}, \quad \mathbf{a}_{\alpha} \times \mathbf{k}=-\mathbf{k} \times \mathbf{a}_{\alpha}=\mathbf{a}^{\beta} \varepsilon_{\beta \alpha}, \mathbf{I}_{\mathrm{s}} \times \mathbf{I}_{\mathrm{s}}=\mathbf{a}_{\alpha} \mathbf{k} \mathbf{a}_{\beta} \varepsilon^{\alpha \beta}=-\boldsymbol{\varepsilon}+\boldsymbol{\varepsilon}_{\mathrm{s}} \mathbf{k}+\mathbf{k} \boldsymbol{\varepsilon}_{\mathrm{s}}$

The symmetric curvature dyadic $\mathbf{b}$ is a measure of the change of $\mathbf{k}$ with changes of $\mathbf{R}$ :

$\mathbf{b}=-\frac{\partial \mathbf{k}}{\partial \mathbf{R}}=-\nabla_{\mathrm{s}} \mathbf{k}, \nabla_{\mathrm{s}}\left({ }^{*}\right)=\mathbf{I}_{\mathrm{s}} \cdot \nabla\left(^{*}\right)=\frac{\partial\left({ }^{*}\right)}{\partial \mathbf{R}}=\mathbf{a}_{\alpha} \frac{\partial\left(^{*}\right)}{\partial \mathbf{u}^{\alpha}}$

where $\nabla_{\mathrm{s}}\left({ }^{*}\right)$ is the surface gradient. The components of $\mathbf{b}$ obey

$b^{\gamma \delta}=a^{\delta \beta} b_{\beta}^{\gamma}, b_{\beta}^{\gamma}=a^{\gamma \alpha} b_{\alpha \beta}$

The average curvature $\mathrm{H}$ and the Gaussian or total curvature $\mathrm{K}$ are :

$2 \mathrm{H}=\mathbf{I}_{\mathrm{s}}: \mathbf{b}=-\nabla_{\mathrm{s}} \cdot \mathbf{k}=-\mathbf{a}^{\alpha} \cdot \frac{\partial \mathbf{k}}{\partial \mathrm{u}^{\alpha}}=\mathrm{b}_{\alpha}^{\alpha}=\left(\mathrm{c}_{1}+\mathrm{c}_{2}\right)$ 
$\mathrm{K}=-\frac{1}{2} \boldsymbol{\varepsilon}_{\mathrm{s}}:\left(\mathbf{b} \cdot \boldsymbol{\varepsilon}_{\mathrm{s}} \cdot \boldsymbol{\varepsilon}_{\mathrm{s}}\right)=\frac{1}{2} \varepsilon^{\alpha \beta} \varepsilon^{\gamma \delta} \mathrm{b}_{\alpha \gamma} \mathrm{b}_{\beta \delta}=\left(\mathrm{c}_{1} \mathrm{c}_{2}\right)$

where $c_{1}$ and $c_{2}$ are the radius of curvature. The relation between $\mathrm{K}$ and $\mathrm{H}$ is:

$\mathrm{K}=2 \mathrm{H}^{2}-\frac{1}{2} \mathbf{b}: \mathbf{b}$

The curvature tensor $\mathbf{b}$ can be decomposed into a trace $\left(\mathrm{HI}_{\mathrm{s}}\right)$ and a deviatoric curvature (Dq ):

$\mathbf{b}=\mathrm{HI} \mathbf{I}_{\mathrm{s}}+\mathrm{Dq}, \quad \mathbf{I}_{\mathrm{s}}: \mathbf{I}_{\mathrm{s}}=\mathbf{q}: \mathbf{q}=2, \quad \mathbf{I}_{\mathrm{s}}: \mathbf{q}=0$

where $D$ is the deviatoric curvature is:

$\mathrm{D}=\frac{1}{2}\left(\mathrm{c}_{1}-\mathrm{C}_{2}\right), \mathrm{D}^{2}=\mathrm{H}^{2}-\mathrm{K}=\frac{1}{2} \mathbf{b}: \mathbf{b}-\mathrm{H}^{2}$

and where in the principal coordinates $\mathbf{q}$ reads:

$\mathbf{q}=\left(\begin{array}{cc}1 & 0 \\ 0 & -1\end{array}\right)$ 


\section{Appendix B: Membratodynamic-Viscoelastic Rey Model (MDRM)}

Using cylindrical coordinates $(r, \phi, z)$, the velocity field $\mathbf{V}$ in the viscoelastic fluid phase is: $\mathbf{V}=\left(0,0, \mathrm{~V}_{\mathrm{z}}(\mathrm{r}, \mathrm{t})\right)$, where $\partial \mathrm{V}_{\mathrm{z}}(\mathrm{r}, \mathrm{t}) / \partial \mathrm{z}=0$

\section{(i) Stress and kinematic tensors}

The stress $t$, velocity gradient and the rate of deformation tensors are given by:

$$
\boldsymbol{\sigma}=\left(\begin{array}{ccc}
\sigma_{\mathrm{rr}} & 0 & \sigma_{\mathrm{rz}} \\
0 & \sigma_{\theta \theta} & 0 \\
\sigma_{\mathrm{zr}} & 0 & \sigma_{\mathrm{zz}}
\end{array}\right) ; \nabla \mathbf{V}=\dot{\gamma}_{\mathrm{rz}}\left(\begin{array}{ccc}
0 & 0 & 1 \\
0 & 0 & 0 \\
0 & 0 & 0
\end{array}\right) ; 2 \mathbf{D}=\dot{\gamma}_{\mathrm{rz}}\left(\begin{array}{ccc}
0 & 0 & 1 \\
0 & 0 & 0 \\
1 & 0 & 0
\end{array}\right)
$$

\section{(ii) Constitutive equation}

Upper-convective Maxwell equation (UCME) is given by the following partial differential equation:

$\rho\left(\frac{\partial}{\partial \mathrm{t}}+\mathbf{V} \cdot \nabla\right) \mathbf{V}=-\nabla \mathrm{P}_{\mathrm{i}}(\mathrm{t})+\nabla \cdot \boldsymbol{\sigma}+\rho \mathbf{g} ; \boldsymbol{\sigma}+\lambda_{\mathrm{i}} \boldsymbol{\sigma}=2 \eta_{\mathrm{i}} \mathbf{D} ; \mathrm{i}=\{\mathrm{t}, \mathrm{b}\}$

And the upper-convected time derivative is given by:

$\stackrel{\nabla}{\boldsymbol{\sigma}}=\frac{\partial}{\partial \mathrm{t}} \boldsymbol{\sigma}+\mathbf{V} \cdot \nabla \boldsymbol{\sigma}-\left\{\nabla \mathbf{V}^{\mathrm{T}} \cdot \boldsymbol{\sigma}+\boldsymbol{\sigma} \cdot \nabla \mathbf{V}\right\}$

In equation (B-2) $\rho$ is the density of the system, $\left\{\lambda_{i}, \eta_{i}\right\}$ are the material properties of the two viscoelastic liquid (bottom and top) and $\mathbf{g}$ is the acceleration of the gravitational forces.

(iii) Shear stress, axial velocity and volumetric flow

Neglecting inertial mechanism and using the rz component of the UCME into the momentum equation, the following expression for the shear stress and the axial velocity is found: 
$\sigma_{\mathrm{rz}}(\mathrm{r}, \mathrm{t})=\frac{\mathrm{r}}{2}\left(\frac{\partial \mathrm{P}_{\mathrm{i}}(\mathrm{t})}{\partial \mathrm{z}}\right) \Rightarrow \mathrm{V}_{\mathrm{z}}(\mathrm{r}, \mathrm{t})=-\frac{\mathrm{a}^{2}}{4 \eta_{\mathrm{i}}}\left(1-\left(\frac{\mathrm{r}}{\mathrm{a}}\right)^{2}\right)\left(1+\lambda_{\mathrm{i}} \frac{\partial}{\partial \mathrm{t}}\right) \frac{\partial \mathrm{P}_{\mathrm{i}}(\mathrm{t})}{\partial \mathrm{z}}$

In equations $(B-5,6)$ the fact that the shear stress must be finite and the non-slip condition are considered. Finally, after a double integration of the velocity and after a time integration of the axial pressure, the following expressions are obtained:

$-\frac{8 \eta_{\mathrm{i}} \mathrm{Q}(\mathrm{t})}{\pi \mathrm{a}{ }^{4} \lambda_{\mathrm{i}}}=\left(\frac{1}{\lambda_{\mathrm{i}}}+\frac{\partial}{\partial \mathrm{t}}\right) \frac{\partial \mathrm{P}_{\mathrm{i}}(\mathrm{z}, \mathrm{t})}{\partial \mathrm{z}} \Rightarrow \frac{\mathrm{dP} \mathrm{P}_{\mathrm{i}}(\mathrm{z}, \mathrm{t})}{\mathrm{dz}}=\mathrm{e}^{-\frac{\mathrm{t}}{\lambda_{\mathrm{i}}}}\left\{\frac{8 \eta_{\mathrm{i}}}{\pi \mathrm{a}^{4} \lambda_{\mathrm{i}}} \int_{0}^{\mathrm{t}} \mathrm{Q}(\xi) \mathrm{e}^{\frac{\xi}{\lambda_{i}}} \mathrm{~d} \xi+\mathrm{C}_{\mathrm{i}}(\mathrm{z})\right\}$

In equation $(\mathrm{B}-8) \mathrm{C}_{\mathrm{i}}(\mathrm{z})$ is a constant that depend of the axial coordinate.

\section{(iv) Membrane pressure top and the bottom}

Integrating (B-8) with respect to the axial coordinate and using the boundary conditions for the top and the bottom $\mathrm{P}_{\mathrm{t}}(\mathrm{z}=2 \mathrm{~L}, \mathrm{t})=\mathrm{P}_{0}$ and $\mathrm{P}_{\mathrm{b}}(\mathrm{z}=0, \mathrm{t})=\mathrm{P}_{0}$. The pressure at the top and the bottom are given by:

$\mathrm{P}_{\mathrm{t}}(\mathrm{z}, \mathrm{t})=\frac{8 \eta_{\mathrm{t}}}{\pi \mathrm{a} \lambda_{\mathrm{t}}}\left(\mathrm{e}^{-\frac{\mathrm{t}}{\lambda_{\mathrm{t}}}} \int_{0}^{\mathrm{t}} \mathrm{Q}(\xi) \mathrm{e}^{\frac{\xi}{\lambda_{\mathrm{t}}}} \mathrm{d} \xi\right)(\mathrm{z}-2 \mathrm{~L})+\mathrm{P}_{\mathrm{o}}$

$\mathrm{P}_{\mathrm{b}}(\mathrm{z}, \mathrm{t})=\frac{8 \eta_{\mathrm{b}}}{\pi \mathrm{a}^{4} \lambda_{\mathrm{b}}}\left(\mathrm{e}^{-\frac{\mathrm{t}}{\lambda_{\mathrm{b}}}} \int_{0}^{\mathrm{t}} \mathrm{Q}(\xi) \mathrm{e}^{\frac{\xi}{\lambda_{\mathrm{b}}}} \mathrm{d} \xi\right) \mathrm{z}+\mathrm{P}_{0}$

The pressure difference $\Delta \mathrm{P}(\mathrm{z}, \mathrm{t})=\mathrm{P}_{\mathrm{t}}(\mathrm{z}, \mathrm{t})-\mathrm{P}_{\mathrm{b}}(\mathrm{z}, \mathrm{t})$ is given by the difference between equations (B-9, B-10): 
$\Delta \mathrm{P}(\mathrm{z}, \mathrm{t})=\frac{8}{\pi \mathrm{a}^{4}}\left\{\frac{\eta_{\mathrm{t}}}{\lambda_{\mathrm{t}}}\left(\mathrm{e}^{-\frac{\mathrm{t}}{\lambda_{\mathrm{t}}}} \int_{0}^{\mathrm{t}} \mathrm{Q}(\xi) \mathrm{e}^{\frac{\xi}{\lambda_{\mathrm{t}}}} \mathrm{d} \xi\right)(\mathrm{z}-2 \mathrm{~L})-\frac{\eta_{\mathrm{b}}}{\lambda_{\mathrm{b}}}\left(\mathrm{e}^{-\frac{\mathrm{t}}{\lambda_{\mathrm{b}}}} \int_{0}^{\mathrm{t}} \mathrm{Q}(\xi) \mathrm{e}^{\frac{\xi}{\lambda_{\mathrm{b}}}} \mathrm{d} \xi\right) \mathrm{z}\right\}$

Evaluating the pressure jump $\Delta \mathrm{P}(\mathrm{z}, \mathrm{t})$ at $\mathrm{z}=\mathrm{L}$ and $\mathrm{using}$ the equation that relates the volumetric in terms of the membrane curvature.

$\mathrm{Q}(\mathrm{t})=-\frac{\pi \mathrm{a}^{4}}{2} \frac{\mathrm{dH}(\mathrm{t})}{\mathrm{dt}} \Rightarrow-\frac{2 \mathrm{Q}(\mathrm{t})}{\pi \mathrm{a}^{4}}=\frac{\mathrm{dH}(\mathrm{t})}{\mathrm{dt}}$

the membrane pressure is obtained; $\Delta \mathrm{P}(\mathrm{L}, \mathrm{t})=\left|\mathbf{k} \mathbf{k}: \Delta \mathbf{T}_{\mathrm{b}}\right|(\mathrm{t})$

$\left|\mathbf{k k}: \Delta \mathbf{T}_{\mathrm{b}}\right|(\mathrm{t})=4 \mathrm{~L}\left\{\mathrm{G}_{\mathrm{b}}\left(\mathrm{e}^{-\frac{\mathrm{t}}{\lambda_{\mathrm{b}}}} \int_{0}^{\mathrm{t}} \mathrm{d} \xi \frac{\mathrm{dH}(\xi)}{\mathrm{d} \xi} \mathrm{e}^{\frac{\xi}{\lambda_{\mathrm{b}}}}\right)+\mathrm{G}_{\mathrm{t}}\left(\mathrm{e}^{-\frac{\mathrm{t}}{\lambda_{\mathrm{t}}}} \int_{0}^{\mathrm{t}} \mathrm{d} \xi \frac{\mathrm{dH}(\xi)}{\mathrm{d} \xi} \mathrm{e}^{\frac{\xi}{\lambda_{\mathrm{t}}}}\right)\right\}$

In equation (B-13), the elastic moduli are defined by: $G_{i}=\eta_{i} / \lambda_{i} ; i=\{t, b\}$. Upon substitution of equation (B-13) into equation (9), the following equation is obtained:

$\frac{\mathbb{C E} \mathfrak{I}}{4 \mathrm{~L}}=\mathrm{G}_{\mathrm{b}}\left(\mathrm{e}^{-\frac{\mathrm{t}}{\lambda_{\mathrm{b}}}} \int_{0}^{\mathrm{t}} \mathrm{d} \xi \frac{\mathrm{dH}(\xi)}{\mathrm{d} \xi} \mathrm{e}^{\frac{\xi}{\lambda_{\mathrm{b}}}}\right)+\mathrm{G}_{\mathrm{t}}\left(\mathrm{e}^{-\frac{\mathrm{t}}{\lambda_{\mathrm{t}}}} \int_{0}^{\mathrm{t}} \mathrm{d} \xi \frac{\mathrm{dH}(\xi)}{\mathrm{d} \xi} \mathrm{e}^{\frac{\xi}{\lambda_{\mathrm{t}}}}\right)+\mathrm{MH}(\mathrm{t})$

where $M$ is defined in eqn. 16-d. Applying the Leibnitz rule twice, it is had the following linear differential equation that describes the evolution of the curvature in the system as a function of the applied electric field and the material properties. Multiplying equation (B-14) by the positive exponential $e^{\left(\frac{t}{\lambda_{b}}\right)}$

$\frac{\mathbb{C} \mathfrak{I}}{4 \mathrm{~L}} \mathrm{e}^{\left(\frac{\mathrm{t}}{\lambda_{\mathrm{b}}}\right)} \mathrm{E}=\mathrm{G}_{\mathrm{b}}\left(\int_{0}^{\mathrm{t}} \mathrm{d} \xi \frac{\mathrm{dH}(\xi)}{\mathrm{d} \xi} \mathrm{e}^{\frac{\xi}{\lambda_{\mathrm{b}}}}\right)+\mathrm{G}_{\mathrm{t}}\left(\mathrm{e}^{\left(\frac{\mathrm{t}}{\lambda_{\mathrm{b}}}-\frac{\mathrm{t}}{\lambda_{\mathrm{t}}}\right)} \int_{0}^{\mathrm{t}} \mathrm{d} \xi \frac{\mathrm{dH}(\xi)}{\mathrm{d} \xi} \mathrm{e}^{\frac{\xi}{\lambda_{\mathrm{t}}}}\right)+\mathrm{Me}^{\left(\frac{\mathrm{t}}{\lambda_{\mathrm{b}}}\right)} \mathrm{H}(\mathrm{t})$ 
Taking the time derivative in both side of equation (B-15) and after that multiplying by the negative exponential $e^{-\left(\frac{t}{\lambda_{b}}-\frac{t}{\lambda_{t}}\right)}$, the following equation is obtained

$$
\begin{aligned}
& \frac{1}{\lambda_{\mathrm{b}}} \frac{\mathbb{C} \mathfrak{J}^{\left(\frac{\mathrm{t}}{\lambda_{\mathrm{t}}}\right)}}{4 \mathrm{~L}} \mathrm{E}+\frac{\mathbb{C} \widetilde{J} \mathrm{e}^{\left(\frac{\mathrm{t}}{\lambda_{\mathrm{t}}}\right)}}{4 \mathrm{~L}} \dot{\mathrm{E}}=\left\{\left(\mathrm{G}_{\mathrm{b}}+\mathrm{G}_{\mathrm{t}}\right)+\mathrm{M}\right\} \mathrm{e}^{\frac{\mathrm{t}}{\lambda_{\mathrm{t}}}} \dot{\mathrm{H}}+\mathrm{G}_{\mathrm{t}}\left(\frac{1}{\lambda_{\mathrm{b}}}-\frac{1}{\lambda_{\mathrm{t}}}\right)\left(\int_{0}^{\mathrm{t}} \mathrm{d} \xi \frac{\mathrm{dH}(\xi)}{\mathrm{d} \xi} \mathrm{e}^{\frac{\xi}{\lambda_{\mathrm{t}}}}\right)+ \\
& \left(\frac{1}{\lambda_{\mathrm{b}}}\right) \mathrm{Me}^{\left(\frac{\mathrm{t}}{\lambda_{\mathrm{t}}}\right)} \mathrm{H}
\end{aligned}
$$

Taking the time derivative both sides in the above equation:

$$
\begin{gathered}
\left(\frac{1}{\lambda_{t} \lambda_{b}} E+\left(\frac{1}{\lambda_{b}}+\frac{1}{\lambda_{t}}\right) \dot{E}+\ddot{E}\right) \frac{\mathbb{C} \Im e^{\left(\frac{t}{\lambda_{t}}\right)}}{4 L}=\left(G_{b}+G_{t}\right) e^{\frac{t}{\lambda_{t}}}(\ddot{H})+\left(\frac{G_{b}}{\lambda_{t}}+\frac{G_{t}}{\lambda_{b}}\right) e^{\frac{t}{\lambda_{t}}} \dot{H}+ \\
M\left(\frac{1}{\lambda_{t}}+\frac{1}{\lambda_{b}}\right) \mathrm{e}^{\frac{t}{\lambda_{t}}} \dot{H}+M^{\frac{t}{\lambda_{t}}}(\ddot{H})+M e^{\frac{t}{\lambda_{t}}}\left(\frac{1}{\lambda_{b} \lambda_{t}} H\right)
\end{gathered}
$$

Changing $b$ by $t$ and $t$ by $b$

$$
\begin{gathered}
\left(\frac{1}{\lambda_{t} \lambda_{b}} E+\left(\frac{1}{\lambda_{t}}+\frac{1}{\lambda_{b}}\right) \dot{E}+\ddot{E}\right) \frac{\left.\mathbb{C} \Im e^{\left(\frac{t}{\lambda_{b}}\right.}\right)}{4 L}=\left(G_{b}+G_{t}\right) e^{\frac{t}{\lambda_{b}}}(\ddot{H})+\left(\frac{G_{b}}{\lambda_{t}}+\frac{G_{t}}{\lambda_{b}}\right) \mathrm{e}^{\frac{t}{\lambda_{b}}} \dot{H}+ \\
M\left(\frac{1}{\lambda_{t}}+\frac{1}{\lambda_{b}}\right) \mathrm{e}^{\frac{t}{\lambda_{b}}} \dot{H}+M^{\frac{t}{\lambda_{b}}}(\ddot{H})+M^{\frac{t}{\lambda_{b}}}\left(\frac{1}{\lambda_{b} \lambda_{t}} H\right)
\end{gathered}
$$

Adding both equations (summarizing the model to eliminate any bias) and deleting $\left(\mathrm{e}^{\frac{\mathrm{t}}{\lambda_{\mathrm{t}}}}+\mathrm{e}^{\frac{\mathrm{t}}{\lambda_{\mathrm{b}}}}\right)$ and rewriting terms:

$$
\begin{gathered}
\left(\eta_{\mathrm{b}} \lambda_{\mathrm{t}}+\eta_{\mathrm{t}} \lambda_{\mathrm{b}}+\mathrm{M} \lambda_{\mathrm{t}} \lambda_{\mathrm{b}}\right) \ddot{\mathrm{H}}+\left(\eta_{\mathrm{b}}+\eta_{\mathrm{t}}+\mathrm{M}\left(\lambda_{\mathrm{b}}+\lambda_{\mathrm{t}}\right)\right) \dot{\mathrm{H}}+\mathrm{M}(\mathrm{H}) \\
=\left(\mathrm{E}+\left(\lambda_{\mathrm{t}}+\lambda_{\mathrm{b}}\right) \dot{\mathrm{E}}+\lambda_{\mathrm{t}} \lambda_{\mathrm{b}} \ddot{\mathrm{E}}\right) \frac{\mathbb{C} \mathfrak{I}}{4 \mathrm{~L}}
\end{gathered}
$$

Finally, equation (B-19) is the base of Rey's model given by equations (14, 15, 16a-f). 


\section{Appendix C: Non-Dimensionalization}

\section{(i) Variables}

To solve the LDE given in $(2-4 a, f)$ the following dimensionless variables are proposed for the electrical field, curvature, time, frequency, viscoelastic properties and power.

$$
\begin{gathered}
\overline{\mathrm{E}}=\frac{\mathrm{E}}{\mathrm{E}_{0}} ; \quad \overline{\mathrm{H}}=\mathrm{aH} ; \quad \overline{\mathrm{t}}=\frac{\mathrm{t}}{\Sigma_{\lambda}} ; \overline{\mathrm{w}}=\Sigma_{\lambda} \mathrm{w} ; \overline{\mathrm{G}_{\mathrm{b}}}=\frac{\mathrm{G}_{\mathrm{b}}}{\Sigma_{\mathrm{G}}} ; \overline{\mathrm{G}_{\mathrm{t}}}=\frac{\mathrm{G}_{\mathrm{t}}}{\Sigma_{\mathrm{G}}} \\
\overline{\lambda_{\mathrm{b}}}=\frac{\lambda_{\mathrm{b}}}{\Sigma_{\lambda}} ; \overline{\lambda_{\mathrm{t}}}=\frac{\lambda_{\mathrm{t}}}{\Sigma_{\lambda}} ; \overline{\mathrm{P}}=\mathrm{P} / 2 \pi \mathrm{a}^{2} \mathrm{~L}\left(\frac{\Sigma_{\mathrm{G}}}{\Sigma_{\lambda}}\right) ; \overline{\mathrm{M}}=\frac{\mathrm{M}}{\Sigma_{\mathrm{G}}}
\end{gathered}
$$

Notice that, for equation (C-19), the following restrictions are satisfied:

$$
\overline{\mathrm{G}}_{\mathrm{b}}+\overline{\mathrm{G}}_{\mathrm{t}}=1 ; \bar{\lambda}_{\mathrm{b}}+\bar{\lambda}_{\mathrm{t}}=1
$$

In equations (C-1-9), the characteristic macroscopic force, length, time, elastic force power and membrane elasticity are: (i) Amplitude of the external electrical field, (ii) radius of the pipe, (iii) sum of the viscoelastic times in the bottom and the top of the applied electrical field, (iv) sum of the elastic moduli in the bottom and the top, respectively. The energy (power) is scaled by the ratio between the sum of the elastic moduli and the viscoelastic time multiplied by a characteristic axial and radial length scales (radius of the pipe and axial length). In our system the characteristic time is the sum of the viscoelastic times. This definition allows comparison with the other internal (inertial, viscoelastic times) and external characteristic times (frequency).

\section{(ii) Dimensionless ordinary differential equation}

Multiplying the differential equation for the evolution of the curvature by $b_{0}^{-1}$ and using the dimensionless variables defined in eqns. (C-1-11) and the following dimensionless time operators for the curvature and the input applied electrical field:

$$
\frac{\mathrm{d}^{\mathrm{n}} \mathrm{H}}{\mathrm{dt}^{\mathrm{n}}} \rightarrow \frac{1 / \mathrm{a}}{\left(\mathrm{a}_{1} / \mathrm{a}_{0}\right)^{\mathrm{n}}} \frac{\mathrm{d}^{\mathrm{n}} \overline{\mathrm{H}}}{\mathrm{dt}} ; \frac{\mathrm{d}^{\mathrm{n}} \mathrm{E}}{\mathrm{dt}^{\mathrm{n}}} \rightarrow \frac{\mathrm{E}_{0}}{\left(\mathrm{a}_{1} / \mathrm{a}_{0}\right)^{\mathrm{n}}} \frac{\mathrm{d}^{\mathrm{n}} \overline{\mathrm{E}}}{\mathrm{dt}} ; \mathrm{H} \rightarrow \mathrm{a}^{-1} \overline{\mathrm{H}} ; \mathrm{E} \rightarrow \mathrm{E}_{0} \overline{\mathrm{E}} ; \mathrm{n}=1,2
$$


the dimensionless ordinary differential equation for the curvature takes the following form:

$$
\frac{1}{\mathrm{a}}\left(\frac{\mathrm{b}_{2} / \mathrm{b}_{0}}{\left(\mathrm{a}_{1} / \mathrm{a}_{0}\right)^{2}} \frac{\mathrm{d}^{2}}{\mathrm{dt}^{-2}}+\frac{\mathrm{b}_{1} / \mathrm{b}_{0}}{\mathrm{a}_{1} / \mathrm{a}_{0}} \frac{\mathrm{d}}{\mathrm{dt}}+1\right) \overline{\mathrm{H}}(\mathrm{t})=\mathrm{E}_{0}\left(\frac{\mathrm{a}_{2} / \mathrm{b}_{0}}{\left(\mathrm{a}_{1} / \mathrm{a}_{0}\right)^{2}} \frac{\mathrm{d}^{2}}{\mathrm{dt}^{-2}}+\frac{\mathrm{a}_{1} / \mathrm{b}_{0}}{\mathrm{a}_{1} / \mathrm{a}_{0}} \frac{\mathrm{d}}{\mathrm{dt}^{-2}}+\mathrm{a}_{0} / \mathrm{b}_{0}\right) \overline{\mathrm{E}}(\mathrm{t})
$$

multiplying (C-13) for the pipe radius a and factorizing $\left(a_{0} / b_{0}\right) /\left(1 / a E_{0}\right)$, it is obtained the dimensionless linear differential equations that describes the evolution of the curvature in terms of the applied input electrical field is obtained:

$$
\left(b_{2}^{*} \frac{d^{2}}{d^{-2}}+b_{1}^{*} \frac{d}{d \bar{t}}+1\right) \bar{H}(t)=a_{0}^{*}\left(a_{2}^{*} \frac{d^{2}}{d^{-2}}+\frac{d}{d \bar{t}}+1\right) \bar{E}(t)
$$

In (C-14), the following identifications were done:

$$
a_{0}^{*}=\frac{a_{0} / b_{0}}{1 / a_{0}} ; a_{2}^{*}=\frac{a_{2} / a_{0}}{\left(a_{1} / a_{0}\right)^{2}} ; b_{2}^{*}=\frac{b_{2} / b_{0}}{\left(a_{1} / a_{0}\right)^{2}} ; b_{1}^{*}=\frac{b_{1} / b_{0}}{a_{1} / a_{0}}
$$

The dimensionless combination of these parameters $\left\{b_{2}^{*} ; b_{1}^{*} ; a_{2}^{*} ; a_{0}^{*}\right\}$ describes the principal mechanisms of the system corresponding to: (i) inertia curvature, (ii) bulk-viscous curvature, (iii) inertia in the input electrical field and (iv) flexoelectric, respectively.

\section{(iii) Dimensionless numbers}

\section{a) Flexoelectric}

The first number $a_{0}^{*}$, is related to the amplitude of the perturbation of the macroscopic flexoelectric force and the elasticity of the membrane. According to data extracted from literature [17]: (i) $a=5 \times 10^{-4} \mathrm{~m}$, (ii) $\mathrm{c}_{\mathrm{f}}=20 \times 10^{-18} \mathrm{C}, \mathrm{k}_{\mathrm{c}}=\overline{\mathrm{k}}_{\mathrm{c}}=0$, (iv) $\gamma_{0}=5 \times 10^{-4} \mathrm{~N} / \mathrm{m}$; (v) $\mathrm{E}_{0}=25 \mathrm{mV} / \mathrm{nm}$ and taking the shape factor as $\mathfrak{I}=8 \mathrm{a}^{-2}$, the dimensionless number $\mathrm{a}_{0}^{*}$ is: 


$$
\mathrm{a}_{0}^{*}=\frac{\mathrm{a}_{0} / \mathrm{b}_{0}}{1 / \mathrm{aE}_{0}}=\frac{\mathrm{aE}_{0} \mathrm{c}_{\mathrm{f}} \mathfrak{I}}{2 \gamma_{0}+\left(2 \mathrm{k}_{\mathrm{c}}+\overline{\mathrm{k}}_{\mathrm{c}}\right) \mathfrak{I}}=\frac{\mathrm{aE}_{0} \mathrm{c}_{\mathrm{f}}\left(8 / \mathrm{a}^{2}\right)}{2 \gamma_{0}+\left(2 \mathrm{k}_{\mathrm{c}}+\overline{\mathrm{k}}_{\mathrm{c}}\right)\left(8 / \mathrm{a}^{2}\right)}=4 \frac{\mathrm{E}_{0} \mathrm{c}_{\mathrm{f}}}{\gamma_{0} \mathrm{a}}=0.82 \approx 1 .
$$

This dimensional number $\mathrm{a}_{0}^{*}$ is close to the unit, so for clarity and readability, this dimensionless number is considered equal to the unit, i.e., $\mathrm{a}_{0}^{*} \cong 1$ in this paper.

\section{b) Memory}

The second dimensionless number is associated to the inertia in the input electrical filed and is

given by: $\mathrm{a}_{2}^{*}=\bar{\lambda}_{\mathrm{t}} \bar{\lambda}_{\mathrm{b}}=\bar{\lambda}_{\mathrm{t}}\left(1-\bar{\lambda}_{\mathrm{t}}\right)$. In the above equation, the relation $\bar{\lambda}_{\mathrm{t}}+\bar{\lambda}_{\mathrm{b}}=1$ was used. The dimensional number $\mathrm{a}_{2}^{*}$ describes a parabolic function whose roots are found in zero and the unit. Taking the derivative of $\mathrm{a}_{2}^{*}$ with respect to $\bar{\lambda}_{\mathrm{t}}$ and equaling to zero, the following critical point and the maximum are obtained:

$$
\mathrm{da}_{2}^{*} / \mathrm{d} \bar{\lambda}_{\mathrm{t}}=0 \Rightarrow \bar{\lambda}_{\mathrm{t}}=1 / 2 \Rightarrow \mathrm{a}_{2 \max }^{*}=\mathrm{a}_{2}^{*}\left(\bar{\lambda}_{\mathrm{t}}=1 / 2\right)=1 / 4
$$

The last expression means that the value of the inertial effects in the input dimensionless electrical field is bounded from: $0 \leq \mathrm{a}_{2}^{*} \leq \mathrm{a}_{2 \max }^{*}=1 / 4$.

\section{c) Bulk-Viscous Mechanism}

The second dimensionless number is the total bulk-viscosity and is given by:

$$
\Sigma_{\bar{\eta}}=\overline{\mathrm{G}}_{\mathrm{t}} \bar{\lambda}_{\mathrm{t}}+\overline{\mathrm{G}}_{\mathrm{b}} \bar{\lambda}_{\mathrm{b}}
$$

The values of the maximum and minimum in the total bulk viscosity are completely determined by the value of the memory of the liquid. As an example of this, suppose that $\bar{\lambda}_{\mathrm{t}} \bar{\lambda}_{\mathrm{b}}=\varepsilon$ and using the relation, $\bar{\lambda}_{\mathrm{t}}+\bar{\lambda}_{\mathrm{b}}=1$; we found a quadrative expression for the Maxwell relaxation time, i.e.,

$$
\bar{\lambda}_{t}\left(1-\bar{\lambda}_{t}\right)=\varepsilon \Rightarrow \bar{\lambda}_{t}-\bar{\lambda}_{t}^{2}=\varepsilon \Rightarrow \bar{\lambda}_{t}^{2}-\bar{\lambda}_{t}+\varepsilon=0
$$


The roots of the above quadratic expression is given by:

$$
\bar{\lambda}_{t}^{(+)}=\frac{1+\sqrt{1-4 \varepsilon}}{2}=\frac{1+1-2 \varepsilon+\mathrm{O}\left(\varepsilon^{2}\right)}{2} \cong 1-\varepsilon ; \bar{\lambda}_{\mathrm{t}}^{(-)}=\frac{1-\sqrt{1-4 \varepsilon}}{2}=\frac{1-\left(1-2 \varepsilon+\mathrm{O}\left(\varepsilon^{2}\right)\right)}{2} \cong \varepsilon
$$

Substitution of roots into $\bar{\lambda}_{\mathrm{t}}+\bar{\lambda}_{\mathrm{b}}=1$; the solutions for the quadratic expression are obtained:

$$
\left.\begin{array}{l}
\bar{\lambda}_{\mathrm{b}}^{(+)}=1-\bar{\lambda}_{\mathrm{t}}^{(+)}=\frac{1-\sqrt{1-4 \varepsilon}}{2}=\frac{1-\left(1-2 \varepsilon+\mathrm{O}\left(\varepsilon^{2}\right)\right)}{2} \approx \varepsilon \\
\bar{\lambda}_{\mathrm{b}}^{(-)}=1-\bar{\lambda}_{\mathrm{t}}^{(-)}=\frac{1+\sqrt{1-4 \varepsilon}}{2}=\frac{1+1-2 \varepsilon+\mathrm{O}\left(\varepsilon^{2}\right)}{2} \approx 1-\varepsilon
\end{array}\right\}
$$

Finally the solutions that satisfy the coupled equations: $\left\{\bar{\lambda}_{t} \bar{\lambda}_{b}=\varepsilon ; \bar{\lambda}_{t}+\bar{\lambda}_{b}=1\right\}$ are given by:

$$
\left\{\left(\bar{\lambda}_{t}^{(+)}=1-\varepsilon, \bar{\lambda}_{\mathrm{b}}^{(+)}=\varepsilon\right),\left(\bar{\lambda}_{\mathrm{t}}^{(-)}=\varepsilon, \bar{\lambda}_{\mathrm{b}}^{(-)}=1-\varepsilon\right)\right\}
$$

(i) Positive roots: $\left(\bar{\lambda}_{t}^{(+)}=1-\varepsilon, \bar{\lambda}_{\mathrm{b}}^{(+)}=\varepsilon\right)$

These roots are substituted into the equation for total bulk viscosity and the result is given by:

$$
\Sigma_{\bar{\eta}}^{(+)}=\overline{\mathrm{G}}_{\mathrm{t}}(1-\varepsilon)+\overline{\mathrm{G}}_{\mathrm{b}} \varepsilon=\overline{\mathrm{G}}_{\mathrm{t}}(1-\varepsilon)+\left(1-\overline{\mathrm{G}}_{\mathrm{t}}\right) \varepsilon ; \overline{\mathrm{G}}_{\mathrm{t}} \in[0,1] \subset \mathrm{R}
$$

In the above equation, the relation $\overline{\mathrm{G}}_{\mathrm{t}}+\overline{\mathrm{G}}_{\mathrm{b}}=1$ was used. It is clear that the linear equation $\Sigma_{\bar{\eta}}^{(+)}$, satisfies the following points: (i) $\quad\left\{\bar{G}_{t}=0, \Sigma_{\bar{\eta}}^{(+)}=\varepsilon\right\}$ and (ii) $\left\{\bar{G}_{t}=1, \Sigma_{\bar{\eta}}^{(+)}=1-\varepsilon\right\}$ respectively, which describe a linear function with a positive slope. This means that the bulkviscous dimensionless number for the positive roots is bounded by the following inequality:

$$
\varepsilon=\min \left\{\bar{\lambda}_{\mathrm{t}}^{(+)}, \bar{\lambda}_{\mathrm{b}}^{(+)}\right\}=\Sigma_{\bar{\eta} \min }^{(+)} \leq \Sigma_{\bar{\eta}}^{(+)} \leq \Sigma_{\bar{\eta} \max }^{(+)}=\max \left\{\bar{\lambda}_{\mathrm{t}}^{(+)}, \bar{\lambda}_{\mathrm{b}}^{(+)}\right\}=1-\varepsilon
$$

(ii) Negative roots: $\left(\bar{\lambda}_{\mathrm{t}}^{(-)}=1-\varepsilon, \bar{\lambda}_{\mathrm{b}}^{(-)}=\varepsilon\right)$

The mathematical treatment of the second root, is similar to the first root; thus, the equation for the total viscosity is given by 


$$
\Sigma_{\bar{\eta}}^{(-)}=\overline{\mathrm{G}}_{\mathrm{t}} \varepsilon+\overline{\mathrm{G}}_{\mathrm{b}}(1-\varepsilon)=\overline{\mathrm{G}}_{\mathrm{t}} \varepsilon+\left(1-\overline{\mathrm{G}}_{\mathrm{t}}\right) 1-\varepsilon ; \overline{\mathrm{G}}_{\mathrm{t}} \in[0,1] \subset \mathrm{R}
$$

Applying the same reasoning, the above equation describes a linear function with negative slope

and satisfies the following points: : (i) $\left\{\overline{\mathrm{G}}_{\mathrm{t}}=0, \Sigma_{\bar{\eta}}^{(-)}=1-\varepsilon\right\}$ and (ii) $\left\{\overline{\mathrm{G}}_{\mathrm{t}}=1, \Sigma_{\bar{\eta}}^{(-)}=\varepsilon\right\}$. The maximum and minimum values satisfy the following inequality:

$$
\varepsilon=\min \left\{\bar{\lambda}_{\mathrm{t}}^{(-)}, \bar{\lambda}_{\mathrm{b}}^{(-)}\right\}=\Sigma_{\bar{\eta} \min }^{(-)} \leq \Sigma_{\bar{\eta}}^{(-)} \leq \Sigma_{\bar{\eta} \max }^{(-)}=\max \left\{\bar{\lambda}_{\mathrm{t}}^{(-)}, \bar{\lambda}_{\mathrm{b}}^{(-)}\right\}=1-\varepsilon
$$

Lastly, the maximum and minimum values of the bulk viscous mechanism depend on the value of the maximum value of the product between dimensionless viscoelastic relaxation times at the top and the bottom of the system (memory).

\section{d) Elastic ratio}

Finally the last dimensional number is the elastic ratio which compare to elastic forces, one of them associated to the flexoelectric membrane and the other one to the bulk elasticity of the system (two viscoelastic fluids in the bottom and the top). Two regimes are possible for the elastic ratio $\mathrm{k}$ : (i) $\overline{\mathrm{M}}<<1$; (ii) $\overline{\mathrm{M}}>>1$.

$\mathrm{k}=\frac{\overline{\mathrm{M}}}{1+\overline{\mathrm{M}}}=\overline{\mathrm{M}}(1+\overline{\mathrm{M}})^{-1}=\overline{\mathrm{M}}\left(1-\overline{\mathrm{M}}+\mathrm{O}\left(\overline{\mathrm{M}}^{2}\right)\right) \approx \overline{\mathrm{M}} ; \quad \operatorname{Lim}_{\overline{\mathrm{M}} \rightarrow \infty} \mathrm{k}=\frac{\overline{\mathrm{M}}}{1+\overline{\mathrm{M}}} \rightarrow 1$

In the first case the elasticity of the membrane is neglected with respect to the elasticity of the bulk. In contrast when $\overline{\mathrm{M}}>>1$, the elastic approaches to the unit., so, the dimensionless elastic ratio is bounded by the following values: $0<\mathrm{k}_{\min } \leq \mathrm{k}<\mathrm{k}_{\max }=1$. In this case, the soft membrane elasticity is higher as compared to the bulk mechanism, and the membrane behaves as an elastic solid. 


\section{e) Dimensionless functions}

In general, the two dimensionless parametric functions $\left\{b_{2}^{*}\left(k, \bar{\lambda}_{t} \bar{\lambda}_{b}\right), b_{1}^{*}\left(k, \Sigma_{\bar{\eta}}\right)\right\}$ satisfy the following inequality: $b_{2}^{*}\left(k, \bar{\lambda}_{t} \bar{\lambda}_{b}\right)<b_{1}^{*}\left(k, \Sigma_{\bar{\eta}}\right)$ As a numerical example of the magnitude of these numbers, the next typical parameters for biological fluids (Outer Hair Cell) are used in the regime of linear viscoelasticity for the top and the bottom [34]: (i) $G_{b}=G_{t}=22 P a$; (ii) $\lambda_{\mathrm{b}}=\lambda_{\mathrm{t}}=5 \times 10^{-5} \mathrm{~s} ; \eta_{\mathrm{b}}=\eta_{\mathrm{t}}=\mathrm{G}_{\mathrm{b}} \lambda_{\mathrm{b}}=\mathrm{G}_{\mathrm{t}} \lambda_{\mathrm{t}}=0.0014 \mathrm{~Pa} \mathrm{~s}$ and for the elastic solid membrane $\gamma_{0}=0.005 \mathrm{Nm}^{-1} ; \quad \mathrm{L}=0.1 \mathrm{~m} ; \mathrm{k}_{\mathrm{c}}=\overline{\mathrm{k}}_{\mathrm{c}}=0.001 \mathrm{~Pa}$, the magnitude order of the dimensionless numbers are: $b_{1}^{*}=8.8 \times 10^{4} ; b_{2}^{*}=4.4 \times 10^{4} ; a_{2}^{*}=0.25$ and $k=5.7 \times 10^{-6}$ these values are in concordance with the inequality given in (C-18). 


\section{Appendix D: Dimensionless Energy Requirements}

\section{a) Power}

The power can be calculated as the average of the product between the square of the product between the curvature and the dimensionless frequency, i.e., $\overline{\mathrm{P}}(\overline{\mathrm{w}})=\left\langle\overline{\mathrm{E}}(\overline{\mathrm{t}}) \cdot \frac{\mathrm{d} \overline{\mathrm{H}}(\overline{\mathrm{t}})}{\mathrm{d} \overline{\mathrm{t}}}\right\rangle$, where the brackets represents an integral average and is given by $\langle\cdot\rangle=\frac{1}{\overline{\mathrm{T}}} \int_{0}^{\overline{\mathrm{T}}} \mathrm{dt} ; \overline{\mathrm{T}}=\frac{2 \pi}{\overline{\mathrm{w}}}$. The definition of the curvature is given by the following expression:

$$
\overline{\mathrm{H}}(\overline{\mathrm{t}} ; \overline{\mathrm{w}})=\underbrace{\overline{\mathrm{H}}_{\mathrm{io}}(\overline{\mathrm{w}})}_{\text {In-out-phase }} \cos (\overline{\mathrm{w}} \overline{\mathrm{t}})+\underbrace{\overline{\mathrm{H}}_{\mathrm{oi}}(\overline{\mathrm{w}})}_{\text {Out-in-phase }} \sin (\overline{\mathrm{w}} \overline{\mathrm{t}})
$$

The time derivative of the curvature is given by:

$$
\dot{\overline{\mathrm{H}}}(\overline{\mathrm{t}} ; \overline{\mathrm{w}})=-\overline{\mathrm{w}}_{\mathrm{H}}(\overline{\mathrm{w}}) \sin (\overline{\mathrm{w} t})+\overline{\mathrm{w}}_{\mathrm{H}}(\overline{\mathrm{w}}) \cos (\overline{\mathrm{w} t})
$$

Upon substitution of the above expression into the definition of the power, the following expression is obtained:

$$
\overline{\mathrm{P}}=\left(\overline{\mathrm{w}} \frac{\overline{2 \pi}}{\int_{0}^{2 \pi / \bar{w}}} \cos (\overline{\mathrm{wt}})\left\{-\overline{\mathrm{w}}_{\mathrm{io}}(\overline{\mathrm{w}}) \sin (\overline{\mathrm{wt}})+\overline{\mathrm{w}}_{\mathrm{oi}}(\overline{\mathrm{w}}) \cos (\overline{\mathrm{wt}})\right\} \mathrm{dt}\right.
$$

By the odd properties of the integrals, and using the following variable changes and the integral trigonometry identity: $\mathrm{z}=\overline{\mathrm{wt}} ; \cos ^{2}(\overline{\mathrm{wt}})=\frac{1}{2}(1+\cos (2 \overline{\mathrm{wt}}))$ the following sum of integrals are obtained:

$$
\overline{\mathrm{P}}=\left(\frac{1}{2 \pi}\right)\{-\overline{\mathrm{w}}_{\mathrm{H}} \overline{\mathrm{i}}_{\mathrm{io}}^{2 \pi} \underbrace{\int_{0}^{2 \pi} \sin (\mathrm{z}) \cos (\mathrm{z}) \mathrm{dz}}_{\mathrm{I}_{1}}+\overline{\mathrm{w}}_{\mathrm{H}_{\mathrm{oi}}} \underbrace{\int_{0}^{2 \pi} \cos ^{2}(\mathrm{z}) \mathrm{dz}}_{\mathrm{I}_{2}}\}
$$


Where the integrals $I_{j}: j=1,2$ can be solved by standard methods to obtain:

$$
I_{1}=\int_{0}^{2 \pi} \sin (z) \cos (z) d z=0 ; \quad I_{2}=\int_{0}^{2 \pi} \cos ^{2}(z) d z=\pi
$$

Collecting all the terms, the power contribution is given by:

$$
\overline{\mathrm{P}}\left(\overline{\mathrm{w}} ; \mathrm{b}_{1}^{*}, \mathrm{~b}_{2}^{*}, \mathrm{k}\right)=\frac{1}{2} \overline{\mathrm{w}} \overline{\mathrm{H}}_{\mathrm{oi}}\left(\overline{\mathrm{w}} ; \mathrm{b}_{1}^{*}, \mathrm{~b}_{2}^{*}, \mathrm{k}\right)
$$

The average power, can be expressed as the product between dimensionless frequency $\overline{\mathrm{W}}$ and dimensionless curvature out-in phase curvature module $\overline{\mathrm{H}}_{\text {oi }}$.

\section{b) Membrane-Elastic Energy}

Applying the same procedure as with the power and repeating all the calculations, the membrane elastic energy can be calculated as the average of the square of the product between the curvature and the dimensionless frequency, i.e., $\overline{\mathrm{Em}}=\left\langle\overline{\mathrm{H}}^{2}(\overline{\mathrm{t}})\right\rangle$, where the brackets represents an integral average. Following the same mathematical development as the power, the following expression is obtained:

$$
\overline{\mathrm{Em}}=\left\langle\overline{\mathrm{H}}^{2}(\overline{\mathrm{t}})\right\rangle=\frac{1}{2}\left\{\overline{\mathrm{H}}_{\mathrm{io}}{ }^{2}+\overline{\mathrm{H}}_{\mathrm{oi}}{ }^{2}\right\}=\frac{1}{2} \overline{\mathrm{H}}^{* 2}
$$

Finally, the elastic membrane energy can be expressed as function of the dimensionless parametric function and the elastic ratio.

$$
\overline{\operatorname{Em}}\left(\overline{\mathrm{w}} ; \mathrm{b}_{1}^{*}, \mathrm{~b}_{2}^{*}, \mathrm{k}\right)=\frac{1}{2} \overline{\mathrm{H}}^{* 2}\left(\overline{\mathrm{w}} ; \mathrm{b}_{1}^{*}, \mathrm{~b}_{2}^{*}, \mathrm{k}\right)
$$

